\title{
3D RECONSTRUCTION OF EXPOSED \\ UNDERGROUND UTILITIES USING \\ PHOTOGRAMMETRIC METHODS
}

\author{
by \\ Wensong $\mathrm{Hu}$ \\ B.Sc., Wuhan University, China, 1992
}

A thesis presented to Ryerson University

in partial fulfillment of the

requirements for the degree of

Master of Applied Science

in the Program of Civil Engineering

Toronto, Ontario, Canada, 2005

(C) Wensong $\mathrm{Hu}, 2005$ 
UMI Number: EC53433

INFORMATION TO USERS

The quality of this reproduction is dependent upon the quality of the copy submitted. Broken or indistinct print, colored or poor quality illustrations and photographs, print bleed-through, substandard margins, and improper alignment can adversely affect reproduction.

In the unlikely event that the author did not send a complete manuscript and there are missing pages, these will be noted. Also, if unauthorized copyright material had to be removed, a note will indicate the deletion.

\section{UMI}

UMI Microform EC53433

Copyright 2009 by ProQuest LLC

All rights reserved. This microform edition is protected against unauthorized copying under Title 17, United States Code.

ProQuest LLC

789 East Eisenhower Parkway

P.O. Box 1346

Ann Arbor, MI 48106-1346 


\section{DECLARATION}

I hereby declare that I am the sole author of this thesis.

I authorize Ryerson University to lend this thesis to other institutions or individuals for the purpose of scholarly research.

Wensong $\mathrm{Hu}$<smiles>C=C</smiles>

Department of Civil Engineering

Ryerson University

I further authorize Ryerson University to reproduce this thesis by photocopying or by other means, in total or in part, at the request of other institutions or individuals for the purpose of scholarly research.

Wensong $\mathrm{Hu}$

U

Department of Civil Engineering

Ryerson University 


\section{BORROWER'S PAGE}

Ryerson University requires the signatures of all persons using or photocopying this thesis. Please sign below, and give address and date.

\begin{tabular}{|c|c|c|c|}
\hline Name of Borrowers & Date & Address & Signature \\
\hline & & & \\
\hline & & & \\
\hline & & & \\
\hline & & & \\
\hline & & & \\
\hline & & & \\
\hline & & & \\
\hline & & & \\
\hline & & & \\
\hline & & & \\
\hline & & & \\
\hline & & & \\
\hline
\end{tabular}




\title{
3D RECONSTRUCTION OF EXPOSED UNDERGROUND
}

\section{UTILITIES USING PHOTOGRAMMETRIC METHODS}

\author{
Wensong $\mathrm{Hu}$ \\ Master of Applied Science, 2005 \\ Department of Civil Engineering \\ Ryerson University
}

\begin{abstract}
This thesis addresses the topic of three-dimensional (3D) reconstruction of exposed underground utilities using photogrammetric methods. Research on this topic is mainly motivated by the need for improved information on the location of underground utilities and, thus, to provide reliable information for the management of buried assets.
\end{abstract}

In this thesis, a system of photogrammetric software programs is developed for $3 \mathrm{D}$ reconstruction of underground utilities. Camera calibration programs are used for computing interior elements and lens distortion coefficients of digital cameras and saving them in a lookup table (LUT). The accuracy of calibrated image coordinates satisfies the photogrammetric processing demand. An automatic image point detection method is proposed and achieved in these programs. External orientation programs are used for calculating exterior elements of the digital images. Based on geographic information system (GIS) and global positioning system (GPS) techniques, a new ground control points (GCPs) collection method is proposed and implemented in these programs. A 3D reconstruction program provides corresponding functions to obtain and edit 3D information of underground utilities. Epipolar lines are employed as an assisting tool that helps operators easily find homologous points from different digital images. The study results indicate that photogrammetric methods for reconstructing $3 \mathrm{D}$ information of underground utilities are effective and low cost. 


\section{ACKNOWLEDGEMENTS}

First of all, I would like to express my great appreciation and thanks to my supervisor, Professor Dr. Michael A. Chapman, for his valuable advice and ideas as well as constructive suggestions and productive comments on my thesis. I also thank him for providing me the great opportunity to continue my study in the field of photogrammetry and remote sensing in Canada and to work with him as a research assistant during my years at Ryerson University. I would also like to thank Professor Dr. Jonathan Li, my co-supervisor, for his encouragement, patience and critical guidance. His innovative suggestions and valuable ideas had a great influence on my thesis study. I greatly appreciate their efforts to provide me financial support throughout my course and thesis studies. Without their support, I would never have completed this thesis work.

I would like to acknowledge my research partner, Mark Tulloch. With his help including background investigation, project organization, field work and valid suggestions, this research works effectively and successfully.

This study was sponsored by the City of Toronto under the "Underground Infrastructure Mapping" project. I would like to thank Kevin Tierney in the City of Toronto for his valuable discussions, suggestions and also the purchase of the mapping-related equipment was very much appreciated. 
I also attribute my accomplishment to Professors Dr. Songnian Li, Dr. Bhagwant Persaud, Dr. Mohamed Lachemi and other faculty and staff members in the Department of Civil Engineering, for their help and support. Many thanks go to Leah Stanwyk, Kim Kritzer and Dianne Mendonca for their administrative support. Many thanks are also extended to Desmond Rogan and Domenic Valle for their technical assistance for solving computer and software problems.

The School of Graduate Studies of Ryerson University is acknowledged for providing me the Ryerson Graduate Scholarships and the opportunity to conduct my research at Ryerson University from 2004 to 2005.

Last, but not least, I wish to express my gratitude, from my deepest heart, to my parents for their unconditional love and support, and to my wife, Xu Sun, for her endless love, patience, and encouragement. Without her help and understanding, I would never complete this thesis. 


\section{TABLE OF CONTENTS}

3D RECONSTRUCTION OF EXPOSED UNDERGROUND UTILITIES USING PHOTOGRAMMETRIC METHODS .................................................................... i

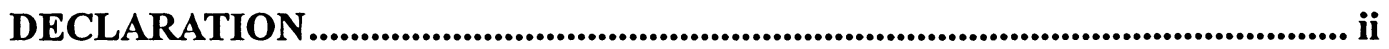

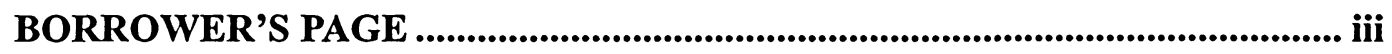

ABSTRACT .......................................................................................................................iv

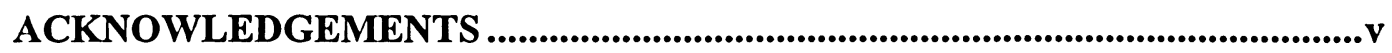

TABLE OF CONTENTS ................................................................................. vii

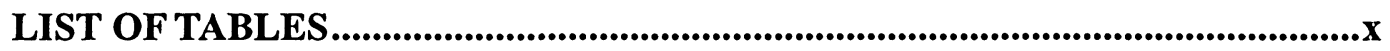

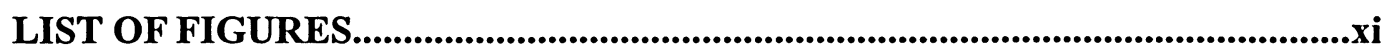

LIST OF ABBREVIATIONS........................................................................... xiii

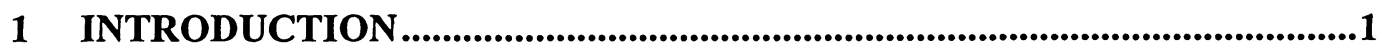

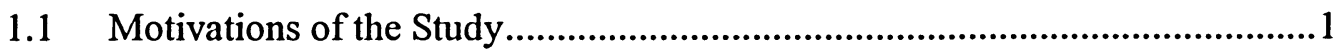

1.2 Problem Statement.....................................................................................

1.3 Objectives of the Study ...............................................................................

1.4 Thesis Organization ......................................................................................6

2 BACKGROUND AND PROBLEMS.......................................................................9

2.1 Ground Penetrating Radar System Approach ................................................9

2.2 Global Position System Method.....................................................................12

2.3 Integrated GPS/GPRS Approach...............................................................

2.4 Conventional Surveying Methods ................................................................ 17

2.5 Subsurface Utilities Engineering Approach.................................................... 18

2.6 Chapter Summary .................................................................................21

3 METHODOLOGY .................................................................................................23 
3.1 Camera Calibration................................................................................23

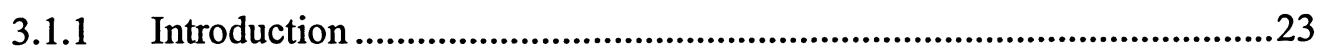

3.1.2 Establishing a Control Pattern ..............................................................26

3.1.3 Automatic Detection of Orientation Points............................................28

3.1.4 Calculation of Interior Elements Using Direct Linear Transformation ..35

3.1.5 Calculation of Lens Distortion Coefficients...........................................38

3.2 External Orientation and GCPs Collection ....................................................39

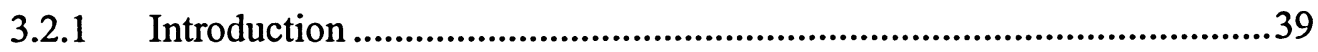

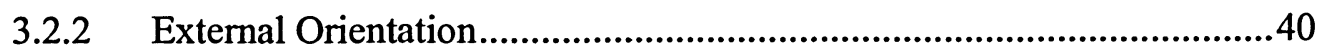

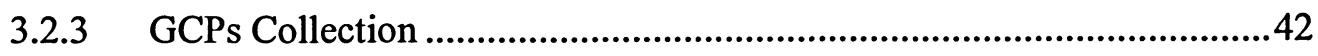

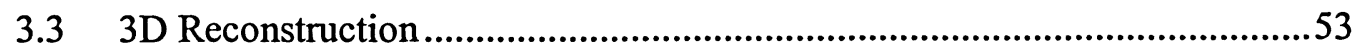

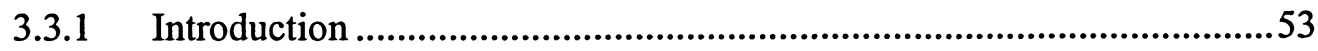

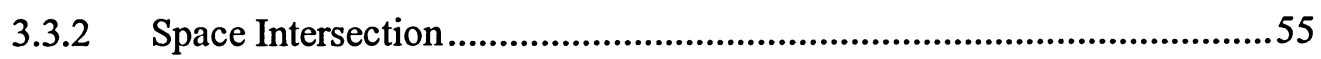

3.3.3 Bundle Adjustment ..........................................................................58

3.3.4 Epipolar Geometry............................................................................61

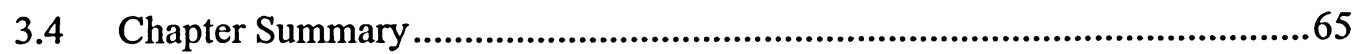

4 CASE STUDY OF CAMERA CALIBRATION ................................................67

4.1 Automatic Detection Program......................................................................67

4.1.1 The Detection of Reference Points ..........................................................68

4.1.2 Obtaining Accurate Positions Using LSM .............................................70

4.1.3 The Combination of Reference Points and GCPs ..................................71

4.2 Camera Calibration and Generation of a Look Up Table ...............................77

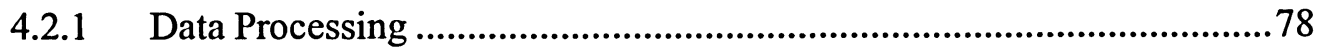

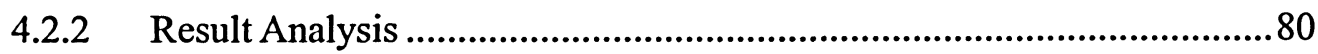

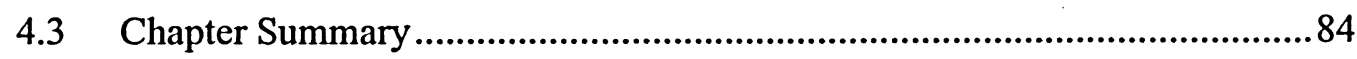

\section{CASE STUDY OF EXTERNAL ORIENTATION AND GCPS}

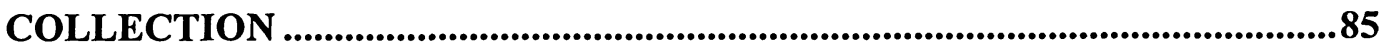

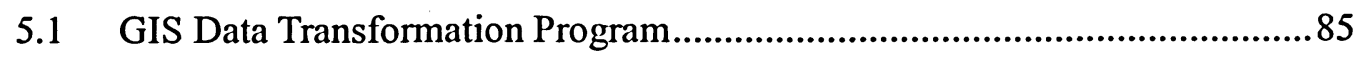

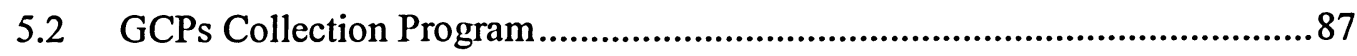


5.3 External Orientation Program ........................................................................91

5.4 Chapter Summary .....................................................................................95

6 CASE STUDY OF 3D RECONSTRUCTION ............................................97

6.1 Model Management Module ......................................................................98

6.2 Object Measurement Module .........................................................................100

6.3 Graphics Editing Module.............................................................................102

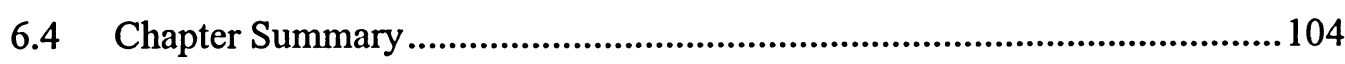

7 CONCLUSIONS AND RECOMMENDATIONS ...............................................107

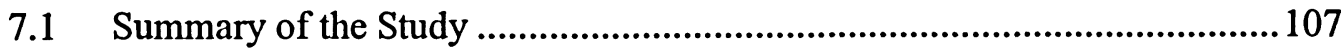

$7.2 \quad$ Limitations of the Study.............................................................................. 109

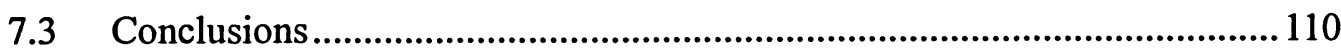

$7.4 \quad$ Recommendations for Future Research....................................................... 112

BIBLIOGRAPHY ........................................................................................................ 113 


\section{LIST OF TABLES}

Table 3-1 List of control points used in camera calibration ....................................28

Table 3-2 Group code value types of DXF format used in this study .......................44

Table 4-1 An example of data set computed by the automatic detection program......77

Table 4-2 The first part of the LUT ............................................................... 82

Table 4-3 The second part of the LUT ..............................................................83 


\section{LIST OF FIGURES}

Figure 2-1 Major activities in subsurface utility engineering (Jeong, et al., 2004).....20

Figure 3-1 Control pattern .27

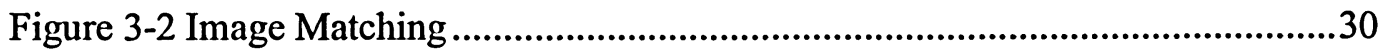

Figure 3-3 Two kinds of fiducial images.........................................................31

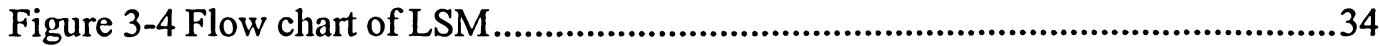

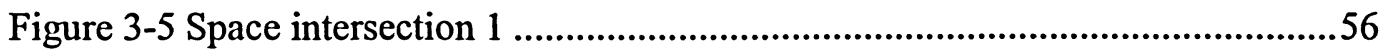

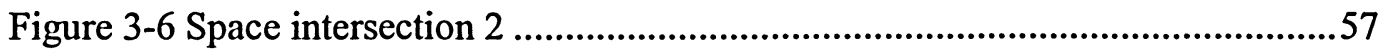

Figure 3-7 Point correspondence geometry....................................................62

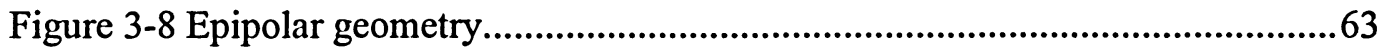

Figure 4-1 The interface of automatic detection program......................................68

Figure 4-2 Flow chart of detecting initial positions of reference points ....................69

Figure 4-3 Flow chart of obtaining accurate positions using LSM. .........................70

Figure 4-4 An example result of automatic detection of reference points ..................71

Figure 4-5 GCP selection window used in determining the relationship between

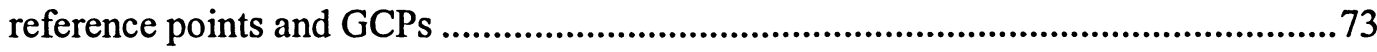

Figure 4-6 Automatically combining reference points and corresponding GCPs.......75

Figure 4-7 An example of combination result ......................................................76

Figure 4-8 The interface of camera calibration program ....................................... 78

Figure 4-9 The data processing flow chart of camera calibration ............................79

Figure 4-10 The calibration result and error vectors.............................................8

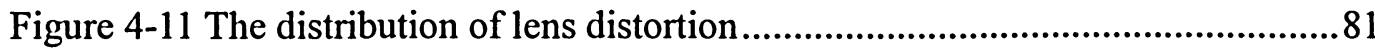


Figure 5-1 Flow chart of GIS data transformation........................................................86

Figure 5-2 The interface of GCPs collection program................................................ 87

Figure 5-3 Zoomed graphics with vertices displayed ................................................... 88

Figure 5-4 Select an intersection as centre point of displayed graphics ......................89

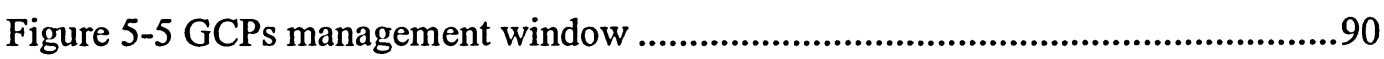

Figure 5-6 The interface of external orientation......................................................92

Figure 5-7 Flow chart of calculating exterior elements ................................................93

Figure 5-8 Results of external orientation..............................................................94

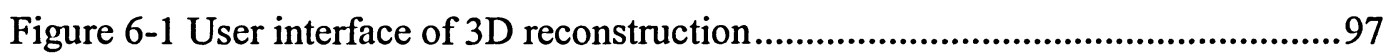

Figure 6-2 Model management window ........................................................98

Figure 6-3 The interface of object measurement module........................................101

Figure 6-4 The legend of three kinds of features.................................................... 103

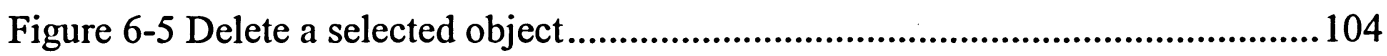




\section{LIST OF ABBREVIATIONS}

2D

$3 \mathrm{D}$

CAD

CADD

DLT

DOT

FHWA

GCPs

GIS

GPR

GPRS

GPS

LSM

LUT

MTM

NMEA

RTK

SUE

VDOT
Two-Dimensional

Three-Dimensional

Computer Aided Design

Computer Aided Design and Drafting

Direct Linear Transformation

Department of Transportation

Federal Highway Administration

Ground Control Points

Geographic Information System

Ground Penetrating Radar

Ground Penetrating Radar System

Global Positioning System

Least Squares Matching

Lookup Table

Modified Transverse Mercator

National Marine Electronics Association

Real Time Kinematic

Subsurface Utilities Engineering

Virginia Department of Transportation 


\section{INTRODUCTION}

\subsection{Motivations of the Study}

The demand for accurate mapping underground utilities is growing rapidly. It involves the systematic location and mapping of buried utilities within a geographic area. The information is recorded into databases for future development and maintenance projects. The management of buried infrastructure becomes important because visual inspection of the underground utilities is not possible. It is difficult to determine or estimate when to rehabilitate or replace utilities. Underground asset management applications assist municipalities in managing their buried infrastructure. Reliable asset management depends heavily on accurate information. Determining the location of buried utilities is one source of information that constitutes good asset management practice (Tulloch and $\mathrm{Hu}, 2005)$.

Furthermore, design and construction projects in areas of concentrated underground utilities require precise utility location information to avoid costly delays, conflicts, redesigns, safety hazards and service disruptions. Using computer-assisted design (CAD) or geographic information system (GIS) technology, underground utility mapping provides a straightforward source of information indicating utility location. Underground utility mapping provides multiple levels of sophisticated, descriptive information about underground utilities. With underground utility mapping, it is 
possible to track a utility from its point of origin to its destination, provide the best routing for the utility line when it was installed as well as periodic maintenance records. Three-dimensional imaging shows the utility from different angles as well as its relationship to other underground objects, minimizing conflicts and allowing for design alternatives.

However, incorrect information is often found on existing underground utility maps. Sometimes utility pipes or cables are relocated during repairs or renovations, but maps are not updated. Sometimes maps represent proposed plans that do not show as-built locations. Occasionally, old maps are simply lost or destroyed. People who fulfill their legal obligation to contact a central utility marking clearinghouse before digging or drilling may feel a false sense of security since lost or mis-mapped utilities generally will not be marked as a result of such a request. The result of digging or drilling in the presence of unknown, unmarked, unmapped, or incorrectly located utilities can be wasted excavation time (and money), expensive damage (and cost), utility downtime (and expenses), and, worst of all, personal injury or death.

This thesis is mainly motivated by the need for improved information on the location of underground utilities, thus, to provide reliable information for buried asset management. Through the solution for accurately mapping the exposed underground utilities that will be mentioned in this study, the risk of construction of underground 
utilities could be reduced greatly. And at the same time, public dollars on infrastructure upgrades will be saved based on the information about the underground utilities.

\subsection{Problem Statement}

The reason that the mapping of underground utilities is gaining more and more attention derives from three main aspects. The first is that the construction risk of underground utilities is increasing due to the uncertainty of the location of the utilities. The ideology of 'out of sight, out of mind' cannot apply to underground utilities any longer. This problem leads to project delays, extra work orders, change orders, construction claims, contingency bidding, loss of service, property damage, and worst of all, injury and death (Anspach, 1996, Tulloch, 2005). Two accidents occurred in Ontario in 2003 due to insufficient of accurate underground information. Eight individuals were killed during these two accidents (Tulloch, 2005). Accurate mapping of the underground utilities is, thus, highly needed for the updating and management of such information.

The second problem is that a considerable outlay of public money for the updating or repairing of the underground utilities will be required due to insufficient and unreliable information related to these utilities. The Municipality of Halton reported that it has spent an average of $\$ 10$ million per year on replacing underground sewer 
and water pipes in the past eight years (Bejon, 2004; Tulloch, 2005). Often, these utilities may be in very good condition at that time. By providing accurate and updated information about underground utilities capital and labour costs will be saved.

The third problem is the disadvantages of existing underground utility mapping methods. The features of the underground utilities are often not detectable using a GPRS approach. The low resolution of GPRS may also produce an incorrect recognition of the underground utility. In addition, it takes a long time for GPS methods to obtain space coordinates of exposed underground utilities. On the other hand, conventional surveying approaches are time and resource intensive as well as limited by environmental factors such as weather. Furthermore, it is very dangerous for surveyors to work in a high-traffic area. SUE is a good approach to obtain spatial information of the underground utilities although the process is very expensive.

The benefits of using photogrammetric methods are numerous. Firstly, this approach is low cost. In particular, the cost of commercial digital cameras is becoming lower and lower. Based upon using these kinds of digital cameras, the cost of digital images is very low. Secondly, a visual record of exposed underground utilities can be permanently saved in the digital images. Thirdly, a safe working environment is provided since most of the data processing operations are performed in the office. 


\subsection{Objectives of the Study}

The main objective of this study is to develop a system for providing three-dimensional (3D) information of the exposed underground utilities using photogrammetric methods. The 3D information of the underground utilities can be used to update the underground utility geo-database and allow for increased public safety, and for better underground asset management.

In order to achieve the above objective, the following key issues are addressed in this study:

- Calibration of the commercial digital camera (Nikon COOLPIX 8800), which will be used in this study. The purpose of camera calibration is to compute the interior elements and lens distortions of the digital camera. The establishing of a control pattern, automatic orientation point detection and the calculation of the camera focal length, principal point and lens distortion coefficients will be included in this section.

- External orientation and ground control points (GCPs) collection. The GIS utility dataset transformation, receiving and locating in the database of the global positioning system (GPS) signal and the selection and recording of the GCPs coordinates will be also included in this section.

- 3D reconstruction of the underground utilities. In this section, the space intersection of target point, the bundle adjustment and the epipolar geometry 
used for homologous points detection will be included.

\subsection{Thesis Organization}

This thesis consists of seven chapters and is organized as follows:

Chapter 1 describes the general goal and problems in underground utilities mapping and sets forth the purpose of this study.

Chapter 2 presents an overview of the methods used in underground utilities mapping. Details are given for the GPS method, photogrammetric method and the subsurface utility engineering (SUE) process.

Chapter 3 proposes a methodology developed and used in this study, including camera calibration, external orientation, GCPs collection and $3 \mathrm{D}$ reconstruction of the underground utilities.

Chapter 4 presents a case study in the use of proposed methodology to perform the calibration of a non-metric digital camera.

Chapter 5 describes the results and discussion of external orientation and GCPs collection. 
Chapter 6 gives the 3D reconstruction results of the underground utilities using the methods mentioned in Chapter 3.

Chapter 7 presents conclusions related to the findings from the study and suggests future work to extend the study in this area. 


\section{BACKGROUND AND PROBLEMS}

Whether installing new underground utilities or repairing old ones, the process of excavation is an important component of civil infrastructure. However, because of the lack of information associated with the exact location and orientation of buried utilities, many problems arise. Some of the construction dangers are the possibility of fatalities, and some may cause considerable economics loss. Hence, accurate detection and mapping of the underground utilities is of utmost importance and has gained more and more attention to the researchers in this area. This chapter attempts to review the relevant research systematically in order to obtain an overview of research trends and to identify the key achievements of various approaches. It is not meant to be an exhaustive review of the literature, but more and indication of the theoretical development of, and problems associated with the mapping of underground utilities.

\subsection{Ground Penetrating Radar System Approach}

Ground penetrating radar (GPR) is a popular technology for mapping subsurface, features, including the public underground infrastructure. Applications of this technology include efficient and precise mapping of buried utilities on a large scale; inspection of the subsurface prior to construction; comparison of "as-builts" to construction plans; inspection of bridge decks and roadbeds; environmental 
monitoring and assessment; near-surface geological assessment; and "non-invasive" archaeology.

GPR involves locating underground infrastructure more reliably and accurately than standard techniques using metal detectors. By providing accurate coverage in 3D, GPR will move the utility industry towards non-invasive management of underground infrastructure, avoiding the hazards and inconvenience of digging. It will also improve construction planning and engineering by showing what lies below the surface before the shovels enters the ground. This technology has incorporated a complete system for underground imaging: 1) an array of antennas to make underground mapping by radar feasible on a large scale; 2) advanced signal processing - using 3D imaging techniques adapted from seismic imaging in oil exploration-to convert radar echoes into 3D underground images; 3) precise positioning of the images relative to ground features by monitoring sensors with a survey total station (e.g. laser theodolite), 4) advanced image processing to extract and display underground features in $3 \mathrm{D}$ and archive the results in computer aided design (CAD) or GIS. GPRs use a high-efficiency GPR array, which can be towed by a vehicle or pushed in front of a modified commercial lawnmower at speeds up to about $1 \mathrm{~km} / \mathrm{h}(30 \mathrm{~cm} / \mathrm{s})$. Standard GPRs use a fixed array of 9 transmitters and 8 receivers. It works by emitting a short pulse of energy into the ground. Echoes are returned from the different interfaces between different materials in the ground. As the antennae emit a "cone" shaped pulse of energy an offset target showing a 
perpendicular face to the radar wave will be "seen" before the antenna passes over it. A resultant characteristic diffraction pattern is, thus, built up in the shape of a hyperbola. A classic target generating such as the diffraction pattern is a pipeline when the antenna is traveling across the line of the pipe. However, it should be pointed out that if the interface between the target and its surrounds does not result in a marked change in velocity then only a weak hyperbola will be seen, if at all.

The advantage of the GPR technology used for underground mapping is that it could overcome the problem of radio-detection methods, which are unable to detect non-metallic buried services including plastic water and gas pipes and clay drainage pipes. At the same time, GPR is a comprehensive and efficient geophysical technology for non-invasive mapping of the underground down to depths of about 6 to 10 feet. By producing a continuous 3D image of the subsurface, GPR can identify the best locations for test pits or vacuum excavation "potholes" to positively identify utilities in place and can fill in gaps between holes. GPR can also be used to quickly develop base maps in areas where records of underground structure are missing or poor. The digital images and maps created by Ground Penetrating Radar System (GPRS) can also be archived for future use in determining possible changes in infrastructure over time by repeated surveys at the same location. But, the GPR devices are very expensive and their penetration capabilities are dependent upon soil conditions. Another major disadvantage of GPRS is the speed. Since the system can only travel at a speed of $1 \mathrm{~km} / \mathrm{h}$, the amount of time needed to collect the underground 
utility information is considerable (Tulloch, 2005).

\subsection{Global Position System Method}

Global Position System (GPS) is a satellite-based navigation system made up of a network of 24 satellites placed into orbit by the U.S. Department of Defence. GPS was originally intended for military applications, but in the 1980s, the government made the system available for civilian use. GPS works in any weather conditions, anywhere in the world, 24 hours a day. There are no subscription fees or setup charges to use GPS. GPS provides specially coded satellite signals that can be processed in a GPS receiver, enabling the receiver to compute position, velocity and time. Based on their parallel multi-channel design, today's GPS receivers are extremely accurate within $15 \mathrm{~m}$ on average.

Underground utility mapping and basemap conversion into GIS and CAD formats have been performed predominantly using photogrammetry or map atlas digitizing methods. Advances in GPS technology during the last few years have allowed new mapping methods that result in comparable costs, shortened duration, and very high quality data collection.

GPS provides a rapid means of field surveying of utility locations. Real-time kinematic (RTK) positioning GPS techniques enable centimeter-level positioning 
instantly at the time of observation. This process of field surveying can replace conventional surveying utility positioning and cost-effectively locate every utility. Moreover, maintaining consistent positioning of additional or relocated facility components can easily be performed to a consistent accuracy specification.

Firstly, utility records from all public and private utility owners should be obstained. With those records in hand, the field crew performs a "top-side" survey-visiting the site to assess whether GPS is the best technique. For example, a site must be relatively open and free of any tree canopies to receive an unobstructed GPS signal. If the surveyors estimate that 60 percent of the features in the area can be located via GPS, it is deemed to be the recommended method.

Secondly, a GPS base station is established, then mobile units carried in backpacks by surveyors are carried to each valve, maintenance hole and other utility features. The surveyor sets up a survey pole with a GPS antenna over each utility feature. Centimeter level positioning can be achieved with one minute of GPS observation data (Gooch, 1997). During this time, the surveyor associates feature codes identifying the feature. Daily production rates vary depending on the density of features. As each point is collected, it shows up on the pre-uploaded digital planimetric base maps, that provides an immediate positional accuracy check for every point. The more planimetric detail carried in the field, such as building 
footprints or sidewalks, the better the opportunity for isolating erroneous GPS positions. For instance, if the surveyor is standing at a fire hydrant 10 feet behind the face of curb and the GPS-observed feature shows up in the middle of the road, the surveyor will identify an obvious need to recollect the point. The newest technological improvements allow surveyors to work smoothly between parts of the site that have a clear GPS signal and those that are obscured from receiving reflected GPS signals by trees or tall buildings. The data collector can be disconnected from the RTK unit, plugged into a total station, and used with conventional techniques-without interrupting the flow of data gathering. Perfected in the last couple of years, this technique eliminates the need to return to the office, download GPS-derived information, upload a data collector and then return to the field. Continuing all field collection with the same data collector provides much smoother transitions between GPS and conventional data areas.

The biggest benefit from using GPS is the durability of the data. In six months, all the paint markings that were applied will be gone. But the GPS derived data and attribution will continuously be available to support new projects numerous times. But, GPS methods can not be used where structures obscure satellite visibility, and conventional surveying must be used at this time. This is a limiting factor in highly developed downtown areas that have tall buildings. The RTK method requires continuous radio transmission between the GPS base station and field unit. At times, interference or loss of signal can cause complications and delays. Mapping of the 
facility network is similar to the photogrammetric approach once the surface features are surveyed. Digitizing is used to connect pipes between the surveyed features guided by plans and atlas sheets. The reference land base map must be highly accurate so that the surveyed locations are positioned relative to road centerlines and other base map features.

\subsection{Integrated GPS/GPRS Approach}

New GPR technology holds promise as a simple, non-invasive approach for sub-surface characterization of proposed underground utility routes. The U.S. Air Force EarthRadar technology--originally developed for detecting and identifying buried unexploded ordnance--is indicating that it can provide the most economical and environmentally friendly tool for subsurface underground utility route investigations. The configuration of the system hardware and signal processing makes it ideal for applications such as mapping subsurface geological features, locating cavities and collapsed features and identifying contaminated ground. It can detect the soil/bedrock interface along the route of proposed underground utilities.

In this method, a high-resolution $( \pm 1 \mathrm{~cm})$ integrated "differential" GPS is used to trigger the GPRS. The global/local coordinates provided by GPS are used in two ways. (1) For location determination and (2) coordinate calculation, which allows surface information to be transformed to the target depth and to aid in 3D image 
reconstruction for discrimination purposes.

A commercially available network analyzer is used in this technology to convey signals via a pair of transmission lines to resonate a suitable antenna. The operating requirements and design specifications of the antenna can change depending on the type, depth, and size of a buried target and the ground conditions. In the process of operating the system, a permanent reference point for the local coordinates GPS is first established. Site characterization tests are performed to calculate the electromagnetic wave speed in the soil. The radar system with its integrated GPS is then set up and all electronics and data files are initialized. Then the system can profile in different colour formats to determine the location and depth of potential buried targets. The 3D images of the potential targets are then constructed.

After the 3D images of the potential targets are obtained, the system could realize the image plots process by changing the data to a frequency domain format. The plots could be either colour plots or greyscale plots. By adding more $2 \mathrm{D}$ view of the buried targets, the system may even generate a global coordinate frame to yield a 3D reconstruction of signal. This resulting reconstruction will more clearly indicate a target object's true dimensions and position of burial.

This technique has provided a simple and relatively low cost solution to the underground utilities detection. It combines the advantages of the GPS and GPRS 
methods into one complete system, and, thus, could provide more accurate underground information. But the disadvantage of this system is also obvious. As a system of the U.S. Air Force, the use of this system is very limited, not all the users would be permitted to use this technology. At the same time, it is not originally designed for underground mapping, so it is convenient to use in some conditions, such as urban areas and it also could not be used when the underground utilities are constructed.

\subsection{Conventional Surveying Methods}

Currently, conventional ground surveying and mapping are still the methods used by some users of underground utility mapping to acquire their data. The advantage of the conventional method is that it can provide required accuracies for collecting underground utility information (Tulloch, 2005). But the disadvantages are significant. Firstly, this method is time and resource intensive since it requires significant data collection and reduction to provide the level of detail necessary for facility location. Secondly, this method is also limited by environmental factors such as weather. With conventional surveying, data collection occurs almost entirely in the field and may require that data collection personnel locate on or near heavily traveled roadways. Additionally, because of extensive in-field data collection, its use is impractical for sizeable projects. And thirdly, once the field data is obtained, a significant amount of processing is necessary before any useful underground utility information is available. 
The result is the passage of a significant amount of time between project inception and final mapping of the underground utilities.

\subsection{Subsurface Utilities Engineering Approach}

Subsurface Utilities Engineering (SUE) is an engineering process that uses new and existing technologies to accurately identify, characterize, and map underground utilities. It is an emerging engineering process that has been proved to be an effective tool to reduce underground utility accidents, damage, utility related claims, and construction delays. This process aims to accurately locate and depict utilities and disseminate the information prior to commencing construction so that conflicts and disasters can be minimized. The practice of SUE has been developed and refined over many years and was systematically put into professional practice in the 1980s (Lew and Anspach, 2000). A state utility engineer in the Virginia Department of Transportation (VDOT) sensed the potential of SUE and allocated $\$ 10,000$ for a trial project in late 1983. This was the first official SUE contract by a state department of transportation (DOT). VDOT reported to the Federal Highway Administration (FHWA) that over $\$ 1$ million in savings to the taxpayer were realized from this project (FHWA, 2002). State DOTs and FHWA since then have taken a leading role in the promotion of SUE, and the term Subsurface Utility Engineering was coined at the 1989 FHWA National Highway Utility Conference. Today, SUE is officially utilized in many agencies, such as the Department of Defence, the Department of Energy, the General Service Administration, as well as many municipalities and engineering firms. 
The SUE process can be categorized into the five distinctive activities as shown in Figure 2-1. It is a combination of geophysics, surveying, civil engineering, and data management. Fieldwork involves three different activities, i.e., subsurface utility designating, subsurface utility locating and surveying. Subsurface utility designating determines the existence and approximate horizontal position of underground utilities using surface geophysical techniques, which include pipe and cable locators, magnetic methods, metal detectors, GPR, acoustic emission methods, etc. In the subsurface utility locating activity, minimally intrusive methods of excavation are used such as vacuum excavation, allowing the determination of the precise horizontal and vertical position of the underground utility line to be documented. Surveying instruments such as levels, staffs and theodolites are typically used for the surveying activities. The GPS is now widely accepted for surveying purposes. Its improved accuracy, e.g., when using real time kinematic technology, and the ease of data transfer to computer aided design and drafting (CADD) and GIS environments have accelerated its use. The data management activity ranges from updating information on existing utility drawings or construction plans to the production of completely new utility maps. In the final engineering service activity, the SUE engineer provides consultation, conflict determinations, and utility coordination and design. 


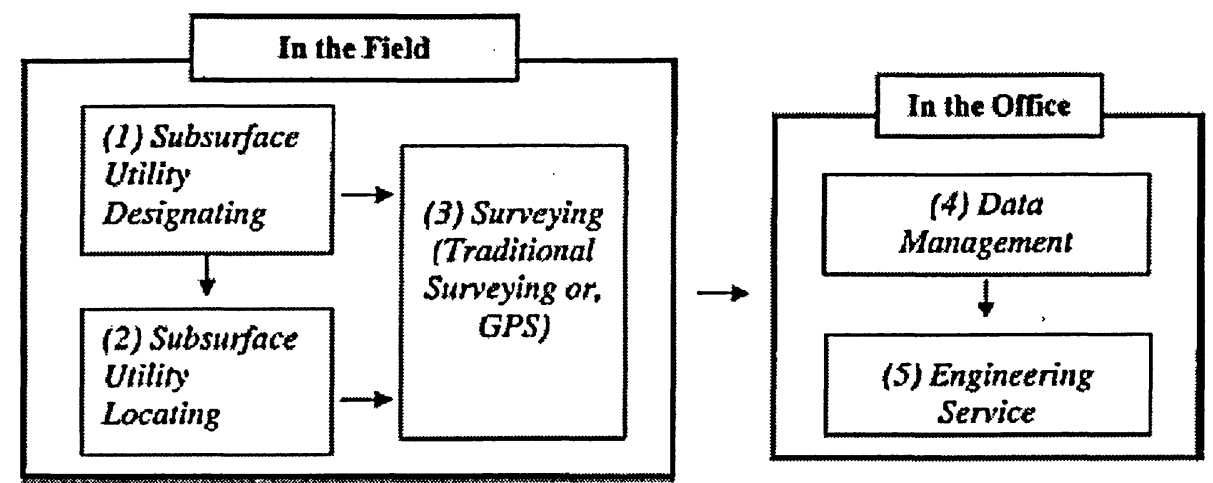

Figure 2-1 Major activities in subsurface utility engineering (Jeong, et al., 2004)

The obvious advantage of SUE is that it can be systematically incorporated during different construction stages. The use of SUE in the preliminary design stage involves all existing utilities designated at the proposed areas of work. It is an approximate horizontal location performed using the surface geophysical methods. The acquired data is transferred on to preliminary plans for the project through a CADD system or GIS. The location of proposed work can be optimized with respect to the horizontal location of the existing utilities. At the final design stage, locations, where conflicts with existing utilities may occur can be identified. And the data collected from the SUE could be used to adjust the final location of the proposed work. The systematic approach allows SUE engineers to narrow down the geographic region where higher quality information is required for the construction project and could then minimize the budget (Jeong, Abraham and Lew, 2004)

There are numerous reports that outline the benefit/cost relationship between SUE and construction projects, and the general benefit/cost ratio is $4: 1$, meaning for every 
dollar spent on SUE, a savings of $\$ 4$ will occur for the overall construction cost (Lew, 1996; Tulloch, 2005). Despite the apparent cost effectiveness of SUE, the process is very expensive. It costs approximately $\$ 1$ per linear foot to accurately map underground utilities, and to perform non-destructive air-vacuum excavation while a borehole costs $\$ 1000$. These costs can quickly accumulate (Tulloch, 2005). At the same time, the lack of professional SUE providers is also a main drawback of SUE method.

\subsection{Chapter Summary}

Detection and identification of buried and submerged structures and utility lines are of interest in many disciplines such as military, law enforcement agencies, construction industry, power suppliers and telecommunication industries. Because of the variable characteristics, degree of saturation, salt and clay mineralogy content of the ground, commercially available detection technology is inadequate for such applications. This chapter presented five popular methods used now in the field of underground utility mapping. The advantages and disadvantages of each method were also described from the view of operation and economic cost. These five methods have provided significant contribution to the application of mapping underground utilities despite some of their disadvantages. The common disadvantages of all these five methods are speed, cost and the fact that they could not be used in the first time of the construction of the underground utilities. 
Based upon the summary of the background and associated problems, this study focuses on the use of photogrammetric method to provide high accuracy and fast 3D reconstruction of the exposed underground utilities. Chapters 3 through 6 will give details on the methodology and its implementation. 


\section{METHODOLOGY}

This chapter describes the methodologies used in this study. Section 3.1 gives conceptual description and methodology of camera calibration. Section 3.2 presents the methodology of external orientation and how to collect GCPs using GPS a receiver and an existing GIS data set. Two 3D reconstruction methods called space intersection and bundle adjustment are introduced in Section 3.3. The concept of epipolar geometry is also provided in this section. Section 3.4 gives a summary of this chapter.

\subsection{Camera Calibration}

\subsubsection{Introduction}

\subsubsection{What Is Camera Calibration?}

In this study, a commercial digital camera is used for obtaining space coordinates of target points. The objective of camera calibration is the determination of the transformation that maps 3D points in a certain scene or object into their corresponding $2 \mathrm{D}$ projections onto the image plane of the camera. This transformation depends on three sets of parameters: (1) internal parameters called interior elements, (2) external parameters called exterior elements, and (3) lens distortion coefficients. There are three interior elements: focal length $(f)$ and the 2D coordinates $\left(x_{0}, y_{0}\right)$ of 
the principal point. The internal geometry of the camera can be described by these elements. The position and orientation of the camera can be determined by 6 independent exterior elements with respect to a given reference system. These include three translation parameters (Xs, Ys, Zs) to determine the position of projection centre and three angular parameters $(\omega, \phi, \kappa)$ to determine the orientation elements of its space axis system in the reference coordinate system. Due to theoretical and technical reasons, it is impossible to manufacture a lens without any distortion. The lens distortion consists of two types of systematic distortions: symmetrical distortion and asymmetrical distortion. Both of them induce a displacement of the image point. Such distortions can be described by distortion coefficients: $\left(k_{0}, k_{1}, k_{2}, k_{3}\right)$ for symmetrical distortion and $\left(p_{1}, p_{2}\right)$ for asymmetrical distortion. After calibrating the camera, all of above parameters can be calculated and can be used in $3 \mathrm{D}$ reconstruction. The transformation can be described by Equations (3-1), (3-2) and (3-3).

$$
\begin{gathered}
x-x_{0}-\Delta x=-f \frac{r_{11}\left(X_{A}-X s\right)+r_{12}\left(Y_{A}-Y s\right)+r_{13}\left(Z_{A}-Z s\right)}{r_{31}\left(X_{A}-X s\right)+r_{32}\left(Y_{A}-Y s\right)+r_{33}\left(Z_{A}-Z s\right)} \\
y-y_{0}-\Delta y=-f \frac{r_{21}\left(X_{A}-X s\right)+r_{22}\left(Y_{A}-Y s\right)+r_{23}\left(Z_{A}-Z s\right)}{r_{31}\left(X_{A}-X s\right)+r_{32}\left(Y_{A}-Y s\right)+r_{33}\left(Z_{A}-Z s\right)} \\
\Delta x=x\left(1-k_{0}-k_{1} r^{2}-k_{2} r^{4}-k_{3} r^{6}\right)+p_{1}\left(r^{2}+2 x^{2}\right)+2 p_{2} x y \\
\Delta y=y\left(1-k_{0}-k_{1} r^{2}-k_{2} r^{4}-k_{3} r^{6}\right)+2 p_{1} x y+p_{2}\left(r^{2}+2 x^{2}\right) \\
R=\left[\begin{array}{lll}
r_{11} & r_{12} & r_{13} \\
r_{21} & r_{22} & r_{23} \\
r_{31} & r_{32} & r_{33}
\end{array}\right]=\left[\begin{array}{ccc}
\cos \kappa & \sin \kappa & 0 \\
-\sin \kappa & \cos \kappa & 0 \\
0 & 0 & 1
\end{array}\right]\left[\begin{array}{ccc}
\cos \phi & 0 & -\sin \phi \\
0 & 1 & 0 \\
\sin \phi & 0 & \cos \phi
\end{array}\right]\left[\begin{array}{ccc}
1 & 0 & 0 \\
0 & \cos \omega & \sin \omega \\
0 & -\sin \omega & \cos \omega
\end{array}\right]
\end{gathered}
$$


where

$x, y: \quad$ image coordinates of control points

$x_{0}, y_{0}: \quad$ the principal point position of a digital camera

$f: \quad$ the focal length of a digital camera

$X_{A}, Y_{A}, Z_{A}: \quad$ space coordinates of control points

$X_{S}, Y_{S}, Z_{s}:$ the photograph position parameters of a digital camera

$\omega, \phi, \kappa: \quad$ the photograph orientation parameters of a digital camera

$\Delta x, \Delta y: \quad$ image point excursion generated by lens distortion

$k_{0}, k_{1}, k_{2}, k_{3}:$ symmetrical distortion coefficients

$p_{1}, p_{2}: \quad$ asymmetrical distortion coefficients

$r: \quad r=\sqrt{x^{2}+y^{2}}$

\subsubsection{Why Is Camera Calibration Needed?}

In this study, a Nikon COOLPIX 8800 digital camera is employed It has a $35-350 \mathrm{~mm}$ 10x Zoom-Nikon ED lens (35mm equivalent) used in capturing different types of digital images. Although the reference focal length can be browsed from INFO.txt file stored in the camera memory, its accuracy is not appropriate for photogrammetric calculations. The location of the principal point and the distortion coefficients according to each focal length are still unknown and needed to be calculated through camera calibration. 


\subsubsection{Strategy of Camera Calibration}

Camera calibration consists of the following procedures:

(1) Establishing a control pattern;

(2) Automatic image points detection;

(3) Direct linear transformation (DLT);

(4) The calculation of lens distortion coefficients and coordinates re-correction;

(5) Repeat (3) and (4) until the average error of homologous points is less than the threshold value (e.g. 0.1 pixel);

(6) Save internal parameters and distortion coefficients into a LUT.

The details about camera calibration are given in the following sections.

\subsubsection{Establishing a Control Pattern}

As mentioned in above section, the objective of camera calibration is the determination of the transformation that maps 3D points of a certain scene or object into their corresponding 2D projections onto the image plane of the camera. Hence, the 3D position $\left(X_{A}, Y_{A}, Z_{A}\right)$ of points must be established as control points before camera calibration. The distribution of control points should be carefully designed. Firstly, all these points should not be located in the same plane as just one image is used in camera calibration. The following paragraphs describe this trivial case.

Suppose $\omega=\phi=\kappa=0$, if all the $\mathrm{Z}$ values of control points equal 0, Equation (3-1) 
can be transformed to Equation (3-4).

$$
\begin{aligned}
& x-x_{0}-\Delta x=f \frac{X_{A}-X s}{Z s} \\
& y-y_{0}-\Delta y=f \frac{Y_{A}-Y_{S}}{Z s}
\end{aligned}
$$

From Equation (3-4), it can be seen that there is a strong correlation between $f$ and Zs. It means whatever $f$ is, we can adjust the $\mathrm{Zs}$ value to get the same result. If all control points are located in the same plane, the same problem will occur again.

Control points also should be distributed in a wide rectangle range that can be projected to most of the image areas. This type of distribution ensures calculations that are much more stable.

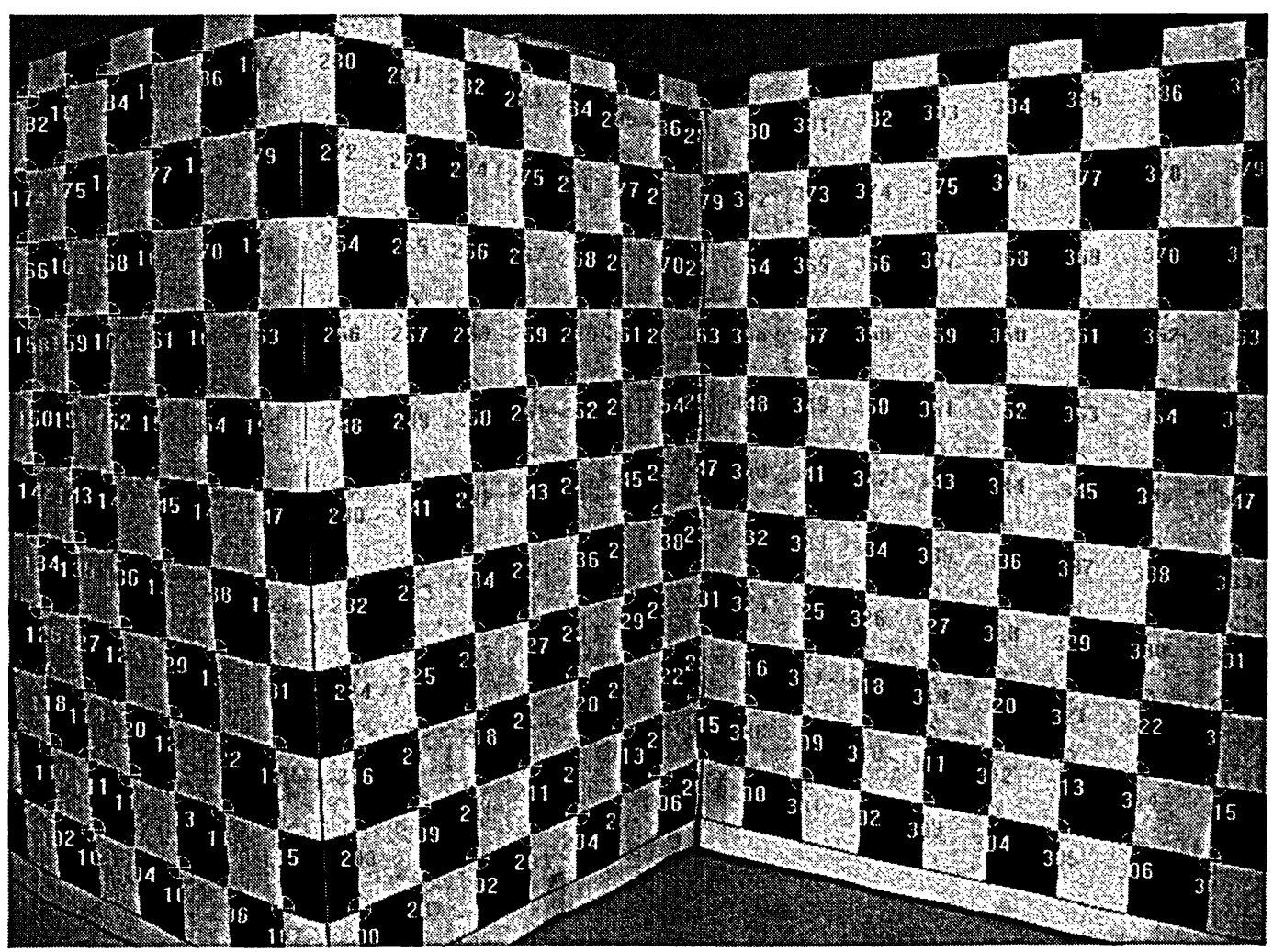

Figure 3-1 Control pattern 
For above reasons, a control pattern shown in Figure 3-1 is used. Cross points of grids are chosen as control points because they are easy to find. Table 3-1 lists part of the point numbers and corresponding 3D coordinates of such points. All of these coordinates were measured by using a total-station.

Table 3-1 List of control points used in camera calibration

\begin{tabular}{cccc}
\hline Point Number & $\mathrm{X}(\mathrm{m})$ & $\mathrm{Y}(\mathrm{m})$ & $\mathrm{Z}(\mathrm{m})$ \\
\hline 102 & -0.2366 & -0.0298 & -0.1693 \\
103 & -0.2048 & -0.0476 & -0.1685 \\
104 & -0.1749 & -0.0615 & -0.1687 \\
105 & -0.1442 & -0.0771 & -0.1686 \\
106 & -0.1134 & -0.0926 & -0.1685 \\
107 & -0.0827 & -0.1066 & -0.1686 \\
110 & -0.2357 & -0.0299 & -0.1352 \\
& & & \\
$\ldots \ldots$ & $\ldots \ldots$. & $\ldots \ldots$. & \\
& & & \\
378 & 0.2639 & 0.0078 & 0.1361 \\
379 & 0.2954 & -0.0079 & 0.1364 \\
380 & 0.0807 & 0.1056 & 0.1699 \\
381 & 0.1114 & 0.0876 & 0.1698 \\
382 & 0.1415 & 0.0719 & 0.1698 \\
383 & 0.1722 & 0.0567 & 0.1699 \\
384 & 0.2028 & 0.0401 & 0.1699 \\
385 & 0.2334 & 0.0244 & 0.1701 \\
386 & 0.2647 & 0.0094 & 0.1706 \\
387 & 0.2964 & -0.0076 & 0.1711 \\
\hline & & &
\end{tabular}

\subsubsection{Automatic Detection of Orientation Points}

From Equation (3-1), the image coordinates of control points also need to be measured. There are two ways to measure them. The first method is to manually measure them by using a digital image processing software program such as 
"Microsoft Paintbrush". This method needs a long time for an operator to complete it. The second method is the automatic detection of orientation points. This method first employs standard patterns to detect the initial positions of target points. Then least squares matching (LSM) is used in measuring the coordinates to sub-pixel accuracy. Finally, target image points are connected to corresponding control points as orientation points.

As the second method is based on automatic searching algorithm, it is easy for users to complete the measuring operation. Hence, the second method is chosen to gain orientation points. The following sections introduce the methodology of automatic orientation point detection.

\subsubsection{Image Matching}

Image matching is used for detecting target points in photogrammetry and remote sensing. It can be defined as the establishment of the correspondence between a fiducial image and a search image. In order to find target part of search image, a template image window is shifted pixel by pixel across a larger search image (see Figure 3-2). 

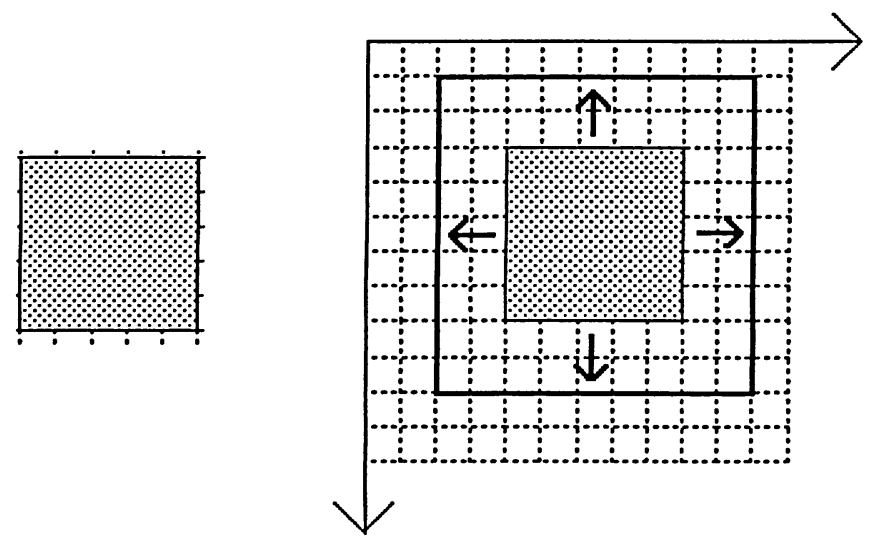

Figure 3-2 Image Matching

Cross correlation is often employed in image matching. The cross correlation coefficient $\rho$ between fiducial image and the corresponding part of the search image is computed according to Equation (3-5).

$$
\rho=\frac{\sum_{r=1}^{R} \sum_{c=1}^{c}(f(r, c)-u)(g(r, c)-v)}{\sqrt{\sum_{r=1}^{R} \sum_{c=1}^{c}(f(r, c)-u)^{2} \sum_{r=1}^{R} \sum_{c=1}^{c}(g(r, c)-v)^{2}}}
$$

where

$f(r, c): \quad$ individual grey values of the fiducial image matrix

$g(r, c): \quad$ individual grey values of the corresponding part of the search image matrix

u: mean grey value of the fiducial image matrix

v: mean grey value of the corresponding part of the search image 
matrix

$\mathrm{R}, \mathrm{C}: \quad$ number of rows and columns of the fiducial and search image matrices

As shown in Figure 3-3, two kinds of images are employed as fiducial images. Target point locates at the centre of fiducial image.
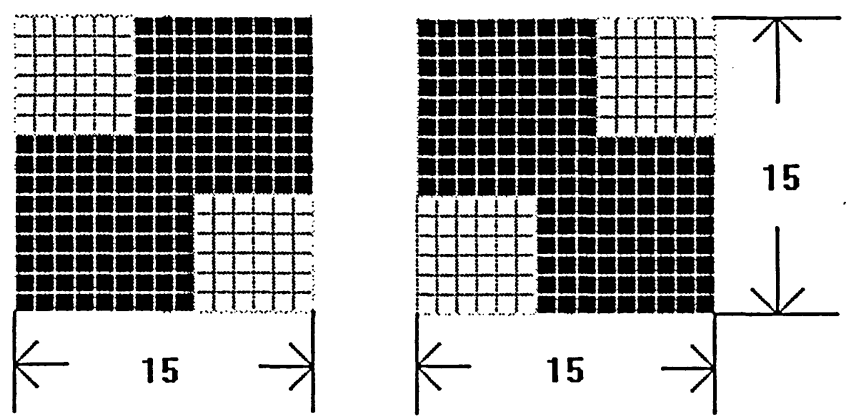

Figure 3-3 Two kinds of fiducial images

Through image matching, initial positions of target points can be established. These positions will be used in LSM as initial values to get more accurate result.

\subsubsection{Least Squares Matching}

LSM is the most accurate image matching technique. Similar to cross correlation, it is based on the similarity of grey values and determines the parameters of an affined transformation (geometric distortion) and illumination and reflectance differences between corresponding patches of two or more images. 
The objective of LSM is to minimize grey value differences between the fiducial window and the matching window by changing the matching window's position and shape, as determined in the adjustment process. The change process stops when the grey value differences between the deformed matching window and the template reach a minimum value. This method not only considers illumination and reflectance differences between the two images but also the geometric distortion of the regular image tessellation caused by unknown orientation parameters, tilted surface patch, a surface patch with relief, etc. The relationship between fiducial window and matching window can be described by Equation (3-6),

$$
f(x, y)=h_{0}+h_{1} g\left(a_{0}+a_{1} x+a_{2} y, \quad b_{0}+b_{1} x+b_{2} y\right)
$$

where

$$
\begin{aligned}
& x, y: \quad \text { image coordinates } \\
& f: \quad \text { grey value of fiducial image } \\
& g: \quad \text { grey value of matching image } \\
& h_{0}, h_{0} \quad \text { illumination and reflectance difference parameters } \\
& a_{0}, a_{1}, a_{2}, b_{0}, b_{1}, b_{2}: \quad \text { geometric distortion coefficients }
\end{aligned}
$$

Equation (3-6) is a nonlinear equation that makes its direct solution difficult and uneconomical. It can be linearized as Equation (3-7)

$$
v=c_{1} d h_{0}+c_{2} d h_{1}+c_{3} d a_{0}+c_{4} d a_{1}+c_{5} d a_{2}+c_{6} d b_{0}+c_{7} d b_{1}+c_{8} d b_{2}-\Delta g
$$


where the unknowns $d h_{0}, d h_{1}, d a_{0}, \ldots, d b_{2}$ are the corrections of the corresponding coefficients, their initial values are:

$$
\begin{aligned}
& h_{0}=0 \\
& h_{1}=1, \\
& a_{0}=0 \\
& a_{1}=1, \\
& a_{2}=0 \\
& b_{0}=0 \\
& b_{1}=0 \\
& b_{2}=1 \\
& c_{1}=1, c_{2}=g_{2} \\
& c_{3}=\frac{\partial g_{2}}{\partial x_{2}} \cdot \frac{\partial x_{2}}{\partial a_{0}} \\
& c_{4}=\frac{\partial g_{2}}{\partial x_{2}} \cdot \frac{\partial x_{2}}{\partial a_{1}} \\
& c_{5}=\frac{\partial g_{2}}{\partial x_{2}} \cdot \frac{\partial x_{2}}{\partial a_{2}} \\
& c_{6}=\frac{\partial g_{2}}{\partial y_{2}} \cdot \frac{\partial y_{2}}{\partial b_{0}} \\
& c_{7}=\frac{\partial g_{2}}{\partial y_{2}} \cdot \frac{\partial y_{2}}{\partial b_{1}} \\
& c_{8}=\frac{\partial g_{2}}{\partial y_{2}} \cdot \frac{\partial y_{2}}{\partial b_{2}} \\
& \Delta g \\
& : \text { grey value difference of the corresponding image point }
\end{aligned}
$$

LSM is very sensitive with respect to the quality of the approximations, requiring, for example, the prior information of correspondences within an accuracy of a few pixels. Thus, it always follows the application of cross correlation and, as a final step, improves the match accuracy (Xie, 2004). Figure 3-4 illustrates the flow chart of LSM. 


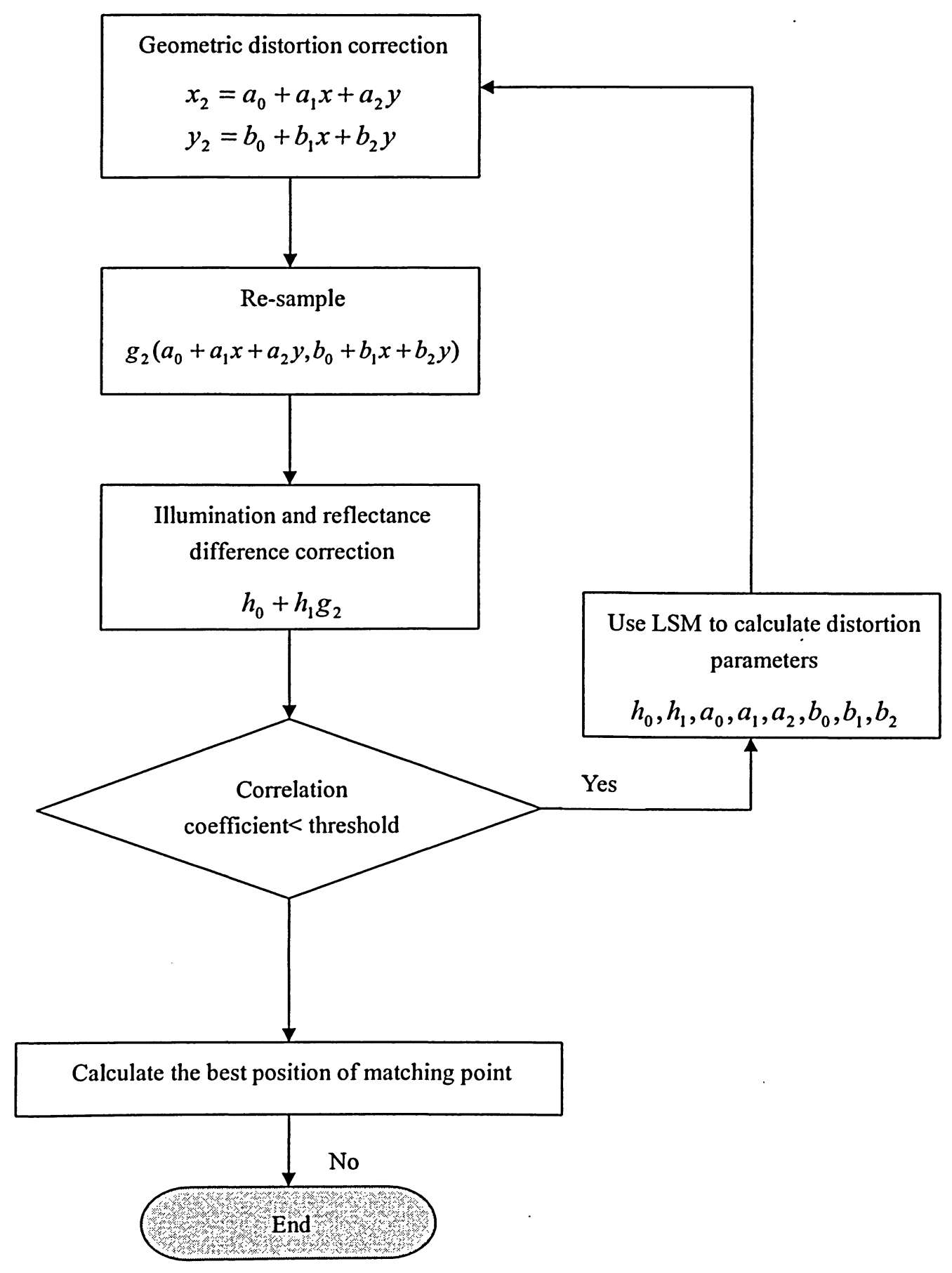

Figure 3-4 Flow chart of LSM 


\subsubsection{Calculation of Interior Elements Using Direct Linear Transformation}

The transformation that maps 3D points of a certain scene or object into their corresponding $2 \mathrm{D}$ projections onto the image plane of the camera can be described by the collinearity equations (see Equation 3-1). Collinearity equations are nonlinear with respect to the variables involved since the rotation matrix $\mathbf{R}$ is based on three angular parameters. Direct solution of this equation is quite impractical, particularly when they are used in conjunction with least squares estimation for redundant cases. There are two methods to solve this problem. The first method is to apply Taylor's series expansion to linearize them. But the requirement of good initial values is its defect. The second method uses a DLT solution. It does not need good initial values. Equation (3-1) can be rearranged as Equation (3-8):

$$
\begin{aligned}
& x-\Delta x=\frac{L_{1} X+L_{2} Y+L_{3} Z+L_{4}}{L_{9} X+L_{10} Y+L_{11} Z+1} \\
& y-\Delta y=\frac{L_{5} X+L_{6} Y+L_{7} Z+L_{8}}{L_{9} X+L_{10} Y+L_{11} Z+1}
\end{aligned}
$$

where

$$
\begin{aligned}
& D=-\left(r_{31} X_{s}+r_{32} Y_{s}+r_{33} Z_{s}\right) \\
& L_{1}=\frac{x_{0} r_{31}-f r_{11}}{D} \\
& L_{2}=\frac{x_{0} r_{32}-f r_{12}}{D} \\
& L_{3}=\frac{x_{0} r_{33}-f r_{13}}{D}
\end{aligned}
$$




$$
\begin{aligned}
& L_{4}=\frac{\left(f r_{11}-x_{0} r_{31}\right) X_{s}+\left(f r_{12}-x_{0} r_{32}\right) Y_{s}+\left(f r_{13}-x_{0} r_{33}\right) Z_{s}}{D} \\
& L_{5}=\frac{y_{0} r_{31}-f r_{21}}{D} \\
& L_{6}=\frac{y_{0} r_{32}-f r_{22}}{D} \\
& L_{7}=\frac{y_{0} r_{33}-f r_{23}}{D} \\
& L_{8}=\frac{\left(f r_{21}-y_{0} r_{31}\right) X_{s}+\left(f r_{22}-y_{0} r_{32}\right) Y_{s}+\left(f r_{23}-y_{0} r_{33}\right) Z_{s}}{D} \\
& L_{9}=\frac{r_{31}}{D} \\
& L_{10}=\frac{r_{32}}{D} \\
& L_{11}=\frac{r_{33}}{D}
\end{aligned}
$$

Coefficients $L_{1}$ to $L_{11}$ in (3-8) are the DLT parameters that reflect the relationships between the object-space reference frame and the image-plane reference frame. The relationship between $k_{i}$ and $\Delta x, \Delta y$ is defined in Equation (3-2). Since there are correlations between $k_{i}$ and $L_{i}$, and $\Delta x, \Delta y$ are very small, the calculation of $k_{i}$ and $L_{i}$ should be separated into two steps. The first step is solving the transformation parameters $L_{i}$. The second step is calculating distortion parameters $k_{i}$ and $p_{i}$. For this reason, Equation (3-8) can be transformed to Equations (3-10)

$$
\begin{aligned}
& L_{1} X+L_{2} Y+L_{3} Z+L_{4}+0 \cdot\left(L_{5}+L_{6}+L_{7}+L_{8}\right)-x\left(L_{9} X+L_{10} Y+L_{11} Z\right)=x \\
& 0 \cdot\left(L_{1}+L_{2}+L_{3}+L_{4}\right)+L_{5} X+L_{6} Y+L_{7} Z+L_{8}-y\left(L_{9} X+L_{10} Y+L_{11} Z\right)=y
\end{aligned}
$$


These equations are linear equations that can be directly solved. The calculation of $L_{i}$ is not our objective. Our purpose is getting internal and external parameters. All of them can be calculated from $L_{i}$.

\subsubsection{Calculation of Camera Position and the Principal Point}

From Equation (3-9)

$$
\begin{aligned}
& L_{1} X_{s}+L_{2} Y_{s}+L_{3} Z_{s}=-L_{4} \\
& L_{5} X_{s}+L_{6} Y_{s}+L_{7} Z_{s}=-L_{8} \\
& L_{9} X_{s}+L_{10} Y_{S}+L_{11} Z_{s}=-1
\end{aligned}
$$

Similarly, from Equation (3-9)

$$
\begin{aligned}
& L_{9}^{2}+L_{10}^{2}+L_{11}^{2}=\frac{r_{31}^{2}+r_{33}^{2}+r_{33}^{2}}{D^{2}}=\frac{1}{D^{2}} \\
& D^{2}=\frac{1}{L_{9}^{2}+L_{10}^{2}+L_{11}^{2}}
\end{aligned}
$$

and

$$
\begin{aligned}
& \left(D L_{1}\right)\left(D L_{9}\right)+\left(D L_{2}\right)\left(D L_{10}\right)+\left(D L_{3}\right)\left(D L_{11}\right) \\
& =x_{0}\left(r_{31}^{2}+r_{32}^{2}+r_{33}^{2}\right)-f\left(r_{11} r_{31}+r_{12} r_{32}+r_{13} r_{33}\right)=x_{0} \\
& \left(D L_{5}\right)\left(D L_{9}\right)+\left(D L_{6}\right)\left(D L_{10}\right)+\left(D L_{7}\right)\left(D L_{11}\right) \\
& =y_{0}\left(r_{31}^{2}+r_{32}^{2}+r_{33}^{2}\right)-f\left(r_{21} r_{31}+r_{22} r_{32}+r_{23} r_{33}\right)=y_{0} \\
& x_{0}=D^{2}\left(L_{1} L_{9}+L_{2} L_{10}+L_{3} L_{11}\right)=\frac{L_{1} L_{9}+L_{2} L_{10}+L_{3} L_{11}}{L_{9}^{2}+L_{10}^{2}+L_{11}^{2}} \\
& y_{0}=D^{2}\left(L_{5} L_{9}+L_{6} L_{10}+L_{7} L_{11}\right)=\frac{L_{5} L_{9}+L_{6} L_{10}+L_{7} L_{11}}{L_{9}^{2}+L_{10}^{2}+L_{11}^{2}}
\end{aligned}
$$

Where $x_{0}$ and $y_{0}$ represent the $\mathrm{x}$ and $\mathrm{y}$ coordinates, respectively, of the principal point. 


\subsubsection{Calculation of Focal Length and Rotation Matrix}

From Equation (3-9)

$$
\begin{aligned}
& R=\left[\begin{array}{lll}
r_{11} & r_{12} & r_{13} \\
r_{21} & r_{22} & r_{23} \\
r_{31} & r_{32} & r_{33}
\end{array}\right]=\left[\begin{array}{ccc}
\frac{x_{0} L_{9}-L_{1}}{f} & \frac{x_{0} L_{10}-L_{2}}{f} & \frac{x_{0} L_{11}-L_{3}}{f} \\
\frac{y_{0} L_{9}-L_{5}}{f} & \frac{y_{0} L_{10}-L_{6}}{f} & \frac{y_{0} L_{11}-L_{7}}{f} \\
L_{9} & L_{10} & L_{11}
\end{array}\right] \\
& f^{2}=D^{2}\left[\left(x_{0} L_{9}-L_{1}\right)^{2}+\left(x_{0} L_{10}-L_{2}\right)^{2}+\left(x_{0} L_{11}-L_{3}\right)^{2}\right] \\
& =\frac{\left(x_{0} L_{9}-L_{1}\right)^{2}+\left(x_{0} L_{10}-L_{2}\right)^{2}+\left(x_{0} L_{11}-L_{3}\right)^{2}}{L_{9}^{2}+L_{10}^{2}+L_{11}^{2}}
\end{aligned}
$$

$D$ can be either positive or negative while calculating rotation matrix. Firstly, use the positive value first and compute the determinant of the transformation matrix obtained. If the determinant is positive (i.e., a right-handed system), $D$ must be positive and the current matrix is acceptable for further use. If the determinant is negative (left-handed), $D$ must be negative. Three angular parameters can be computed from the nine elements of the rotation matrix although it is not necessary.

\subsubsection{Calculation of Lens Distortion Coefficients}

As mentioned in Section 3.1.1, the lens distortion consists of two types of distortions: symmetrical distortion and asymmetrical distortion. The effect of above distortions can be described in Equation (3-2). Since $k_{i}$ is a function of the focal length, it 
should be separately computed from the focal length. Hence, internal parameters (including focal length) and external parameters should be computed first. Then compute distortion coefficients and correct the image coordinates for target points. After that, repeat these steps until the correction of focal length is smaller than a given threshold (e.g., 0.1 pixel).

\subsection{External Orientation and GCPs Collection}

\subsubsection{Introduction}

Equation (3-1) describes the transformation that maps 3D points of a certain scene or object into their corresponding 2D projections onto the image plane of the camera. This Equation also can be used for gaining 3D coordinates of target point if more than two digital images overlap target area and the interior elements, distortion coefficients and exterior elements are known. The interior elements and distortion coefficients are computed from camera calibration and can be used as known parameters. Hence, exterior elements should be calculated first. The inverse computation for the exterior

elements, $X_{s}, Y_{s}, Z_{s}$ and $\phi, \omega, \kappa$ is called external orientation. Section 3.2.2 describes the methodology of external orientation.

From Equation (3-1) at least three points with known ground coordinates are required to determine the six exterior elements. Such points are called GCPs. The image coordinates $(x, y)$ of GCPs can be directly measured in the overlap area of the images. 
Section 3.2.3 presents how to collect GCPs used in this study.

\subsubsection{External Orientation}

Although the DLT can be used for external orientation, at least 6 GCPs are required. In general, it is difficult to obtain enough GCPs. On the contrary, collinearity equation only needs 3 GCPs to calculate 6 unknown parameters. Hence, collinearity equation is chosen for external orientation in this study.

Equation (3-1) is nonlinear and should be linearized by using a Taylor's series expansion. In linearizing them, Equation (3-1) is rewritten as follows:

$$
\begin{aligned}
& F=x_{0}+\Delta x-f \frac{R}{Q}=x \\
& G=y_{0}+\Delta y-f \frac{S}{Q}=y
\end{aligned}
$$

where

$$
\begin{aligned}
& Q=r_{31}\left(X-X_{s}\right)+r_{32}\left(Y-Y_{s}\right)+r_{33}\left(Z-Z_{s}\right) \\
& R=r_{11}\left(X-X_{s}\right)+r_{12}\left(Y-Y_{s}\right)+r_{13}\left(Z-Z_{s}\right) \\
& S=r_{21}\left(X-X_{s}\right)+r_{22}\left(Y-Y_{s}\right)+r_{23}\left(Z-Z_{s}\right)
\end{aligned}
$$

According to Taylor's series, Equation (3-15) can be expressed in linearized form by taking the partial derivatives with respect to the unknowns:

$$
\begin{aligned}
& F_{0}+\left(\frac{\partial F}{\partial \omega}\right) d \omega+\left(\frac{\partial F}{\partial \phi}\right) d \phi+\left(\frac{\partial F}{\partial \kappa}\right) d \kappa+\left(\frac{\partial F}{\partial X_{S}}\right) d X_{S}+\left(\frac{\partial F}{\partial Y_{s}}\right) d Y_{S}+\left(\frac{\partial F}{\partial Z_{S}}\right) d Z_{s}=x \\
& G_{0}+\left(\frac{\partial G}{\partial \omega}\right) d \omega+\left(\frac{\partial G}{\partial \phi}\right) d \phi+\left(\frac{\partial G}{\partial \kappa}\right) d \kappa+\left(\frac{\partial G}{\partial X_{S}}\right) d X_{S}+\left(\frac{\partial G}{\partial Y_{S}}\right) d Y_{S}+\left(\frac{\partial G}{\partial Z_{S}}\right) d Z_{s}=y
\end{aligned}
$$


In Equation (3-17) $F_{0}$ and $G_{0}$ are functions $\mathrm{F}$ and $\mathrm{G}$ of Equation (3-15) evaluated at the initial approximations for the 6 unknown exterior elements; the terms $\left(\frac{\partial F}{\partial \omega}\right),\left(\frac{\partial F}{\partial \phi}\right),\left(\frac{\partial G}{\partial \omega}\right),\left(\frac{\partial G}{\partial \phi}\right)$, etc., are partial derivatives of functions $\mathrm{F}$ and $\mathrm{G}$ with respect to the indicated unknowns evaluated at the initial approximations; and $d \omega, d \phi, d \kappa$, etc., are unknown corrections to be applied to the initial approximations. The units of $d \omega, d \phi$ and $d \kappa$ are radians. Equation (3-17) can be simplified to the following form:

$$
\begin{aligned}
& b_{11} d \omega+b_{12} d \phi+b_{13} d \kappa-b_{14} d X_{s}-b_{15} d Y_{S}-b_{16} d Z_{s}=J+v_{x} \\
& b_{21} d \omega+b_{22} d \phi+b_{23} d \kappa-b_{24} d X_{S}-b_{25} d Y_{S}-b_{26} d Z_{S}=K+v_{y}
\end{aligned}
$$

In Equation (3-18); $\mathrm{J}$ and $\mathrm{K}$ are equal to $\mathrm{x}-F_{0}$ and $\mathrm{y}-G_{0}$, respectively. The coefficients $b_{i j}$ are equal to the partial derivatives. For convenience, these coefficients are given below. Numerical values for these coefficient terms are obtained by using initial approximations for the unknowns.

$$
\begin{aligned}
& b_{11}=\frac{f}{q^{2}}\left[R\left(-r_{33} \Delta Y+r_{32} \Delta Z\right)-Q\left(-r_{13} \Delta Y+r_{12} \Delta Z\right]\right. \\
& b_{12}=\frac{f}{q^{2}}\left[\begin{array}{l}
R(\cos \phi \Delta X+\sin \omega \sin \phi \Delta Y-\cos \omega \sin \phi \Delta Z) \\
-Q(-\sin \phi \cos \kappa \Delta X+\sin \omega \cos \phi \cos \kappa \Delta Y-\cos \omega \cos \phi \cos \kappa \Delta Z
\end{array}\right] \\
& b_{13}=-\frac{f}{q}\left(r_{21} \Delta X+r_{22} \Delta Y+r_{23} \Delta Z\right) \\
& b_{14}=\frac{f}{q^{2}}\left(r_{31} R-r_{11} Q\right) \\
& b_{15}=\frac{f}{q^{2}}\left(r_{32} R-r_{12} Q\right) \\
& b_{16}=\frac{f}{q^{2}}\left(r_{33} R-r_{13} Q\right) \\
& J=x-x_{0}-\Delta x+f \frac{R}{Q}
\end{aligned}
$$




$$
\begin{aligned}
& b_{21}=\frac{f}{q^{2}}\left[S\left(-r_{33} \Delta Y+r_{32} \Delta Z\right)-Q\left(-r_{23} \Delta Y+r_{22} \Delta Z\right]\right. \\
& b_{22}=\frac{f}{q^{2}}\left[\begin{array}{l}
S(\cos \phi \Delta X+\sin \omega \sin \phi \Delta Y-\cos \omega \sin \phi \Delta Z) \\
-Q(-\sin \phi \sin \kappa \Delta X+\sin \omega \cos \phi \sin \kappa \Delta Y-\cos \omega \cos \phi \sin \kappa \Delta Z
\end{array}\right] \\
& b_{33}=-\frac{f}{q}\left(r_{11} \Delta X+r_{12} \Delta Y+r_{13} \Delta Z\right) \\
& b_{14}=\frac{f}{q^{2}}\left(r_{31} S-r_{21} Q\right) \\
& b_{15}=\frac{f}{q^{2}}\left(r_{32} S-r_{22} Q\right) \\
& b_{16}=\frac{f}{q^{2}}\left(r_{33} S-r_{23} Q\right) \\
& K=y-y_{0}-\Delta y+f \frac{S}{Q} \\
& \Delta X, \Delta Y, \text { and } \Delta Z \text { are equal to } X-X_{s}, Y-Y_{s} \text { and } Z-Z_{s}, \text { respectively. }
\end{aligned}
$$

\subsubsection{GCPs Collection}

Traditionally, the ground coordinates of GCPs can be computed through block triangulation or obtained using a high accuracy GPS receiver. Block aerotriangulation is a good method to determine the range of control extension in aerial photogrammetry. But it is difficult to find enough appropriate points in urban areas.

On the other hand, although the ground coordinates of GCPs can be measured by using a high accuracy GPS receiver, GPS signals are often blocked by large structures. Hence, both of above methods are not suitable for obtaining GCPs in urban areas.

A new method to complete GCPs collection is proposed in this study. Firstly, a GIS dataset supported by the City of Toronto that contains the ground coordinates of 
utilities is transformed to special format designed for this study and displayed in a navigating program. Secondly, a commercial GPS receiver that supports a $10 \mathrm{~m}$-accuracy location is employed to get initial positions. Thirdly, the GIS dataset is navigated to the current position and appropriate vertices are chosen as GCPs. The sections below describe the details of this method.

\subsubsection{GIS Dataset Transformation}

The utilities GIS dataset supported by the City of Toronto is made in AutoCAD2005 with "dwg" format. It is difficult to directly extract the GCPs and use them in our external orientation program. Furthermore, not every set of coordinates is useful for GCPs collection. For these reasons, we need to transform this dataset into our special format that discards unnecessary parts of the utilities.

Since the AutoCAD2005 "dwg" format is so complex, we transform it into AutoCAD DXF format. The $\mathrm{DXF}^{\mathrm{TM}}$ format is a tagged data representation of all the information contained in an AutoCAD $^{\circledR}$ drawing file. Tagged data means that an integer number that is called a group code precedes each data element in the file. A group code's value indicates what type of data element follows. This value also indicates the meaning of a data element for a given object (or record) type. Virtually all user-specified information in a drawing file can be represented in DXF format. Group codes define the type of the associated value as an integer, a floating-point number, or a string, 
according to the Table 3-2 of group code ranges.

Table 3-2 Group code value types of DXF format used in this study

\begin{tabular}{|c|c|}
\hline Group code & Description \\
\hline-5 & APP: persistent reactor chain \\
\hline-4 & APP: conditional operator (used only with ssget) \\
\hline-3 & APP: extended data (XDATA) sentinel (fixed) \\
\hline-2 & APP: entity name reference (fixed) \\
\hline-1 & $\begin{array}{l}\text { APP: entity name. The name changes each time a drawing is } \\
\text { opened. It is never saved (fixed) }\end{array}$ \\
\hline 0 & Text string indicating the entity type (fixed) \\
\hline 1 & Primary text value for an entity \\
\hline 2 & Name (attribute tag, block name, and so on) \\
\hline $3-4$ & Other text or name values \\
\hline 5 & Entity handle; text string of up to 16 hexadecimal digits (fixed) \\
\hline 6 & Linetype name (fixed) \\
\hline 7 & Text style name (fixed) \\
\hline 8 & Layer name (fixed) \\
\hline 9 & $\begin{array}{l}\text { DXF: variable name identifier (used only in HEADER section of } \\
\text { the DXF file) }\end{array}$ \\
\hline \multirow[t]{3}{*}{10} & $\begin{array}{l}\text { Primary point; this is the start point of a line or text entity, center } \\
\text { of a circle, and so on }\end{array}$ \\
\hline & $\begin{array}{l}\text { DXF: } X \text { value of the primary point (followed by } Y \text { and } Z \text { value } \\
\text { codes } 20 \text { and 30) }\end{array}$ \\
\hline & APP: 3D point (list of three reals) \\
\hline \multirow[t]{3}{*}{$11-18$} & Other points \\
\hline & $\begin{array}{l}\text { DXF: } X \text { value of other points (followed by } Y \text { value codes } 21-28 \\
\text { and } Z \text { value codes } 31-38 \text { ) }\end{array}$ \\
\hline & APP: 3D point (list of three reals) \\
\hline 20,30 & DXF: $Y$ and $Z$ values of the primary point \\
\hline $21-28,31-37$ & DXF: $Y$ and $Z$ values of other points \\
\hline 38 & DXF: entity's elevation if nonzero \\
\hline 39 & Entity's thickness if nonzero (fixed) \\
\hline $40-48$ & Floating-point values (text height, scale factors, and so on) \\
\hline 48 & $\begin{array}{l}\text { Linetype scale; floating-point scalar value; default value is } \\
\text { defined for all entity types }\end{array}$ \\
\hline 49 & $\begin{array}{l}\text { Repeated floating-point value. Multiple } 49 \text { groups may appear in } \\
\text { one entity for variable-length tables (such as the dash lengths in } \\
\text { the LTYPE table). A } 7 x \text { group always appears before the first } 49 \\
\text { group to specify the table length }\end{array}$ \\
\hline $50-58$ & Angles (output in degrees to DXF files and radians through \\
\hline
\end{tabular}




\begin{tabular}{|c|c|}
\hline & AutoLISP and ObjectARX applications) \\
\hline 60 & $\begin{array}{l}\text { Entity visibility; integer value; absence or } 0 \text { indicates visibility; } 1 \\
\text { indicates invisibility }\end{array}$ \\
\hline 62 & Colour number (fixed) \\
\hline 66 & "Entities follow" flag (fixed) \\
\hline 67 & Space-that is, model or paper space (fixed) \\
\hline 68 & $\begin{array}{l}\text { APP: identifies whether viewport is on but fully off screen; is not } \\
\text { active or is off }\end{array}$ \\
\hline 69 & APP: viewport identification number \\
\hline 70-78 & Integer values, such as repeat counts, flag bits, or modes \\
\hline $90-99$ & 32-bit integer values \\
\hline 100 & $\begin{array}{l}\text { Subclass data marker (with derived class name as a string). } \\
\text { Required for all objects and entity classes that are derived from } \\
\text { another concrete class. The subclass data marker segregates data } \\
\text { defined by different classes in the inheritance chain for the same } \\
\text { object. } \\
\text { This is in addition to the requirement for DXF names for each } \\
\text { distinct concrete class derived from ObjectARX }\end{array}$ \\
\hline 102 & $\begin{array}{l}\text { Control string, followed by " }\{<\text { arbitrary name }>\text { " or " }\} " . \text { Similar to } \\
\text { the xdata } 1002 \text { group code, except that when the string begins } \\
\text { with " }\{\text { ", it can be followed by an arbitrary string whose } \\
\text { interpretation is up to the application. The only other control } \\
\text { string allowed is " }\} \text { " as a group terminator. AutoCAD does not } \\
\text { interpret these strings except during drawing audit operations. } \\
\text { They are for application use }\end{array}$ \\
\hline 105 & Object handle for DIMVAR symbol table entry \\
\hline 1000 & ASCII string (up to 255 bytes long) in extended data \\
\hline 1001 & $\begin{array}{l}\text { Registered application name (ASCII string up to } 31 \text { bytes long) } \\
\text { for extended data }\end{array}$ \\
\hline 1002 & Extended data control string (" $\{$ "or "\}") \\
\hline 1003 & Extended data layer name \\
\hline 1004 & Chunk of bytes (up to 127 bytes long) in extended data \\
\hline 1005 & $\begin{array}{l}\text { Entity handle in extended data; text string of up to } 16 \text { hexadecimal } \\
\text { digits }\end{array}$ \\
\hline \multirow[t]{3}{*}{1010} & A point in extended data \\
\hline & DXF: $X$ value (followed by 1020 and 1030 groups) \\
\hline & APP: 3D point \\
\hline 1020,1030 & DXF: $Y$ and $Z$ values of a point \\
\hline
\end{tabular}

Using these group code and value pairs, a DXF file is organized into sections composed of records, which are composed of a group code and a data item. Each 
group code and value is on its own line in the DXF file. Each section starts with a group code 0 followed by the string SECTION and then a group code 2 and a string indicating the name of the section follows. Each section is composed of group codes and values that define its elements. A section ends with a 0 followed by the string ENDSEC.

There are 7 sections in a DXF file. The overall organization of a DXF file is as followings:

HEADER section. Contains general information about the drawing. It consists of an AutoCAD database version number and a number of system variables. Each parameter contains a variable name and its associated value. The following is an example of the HEADER section of a DXF file:

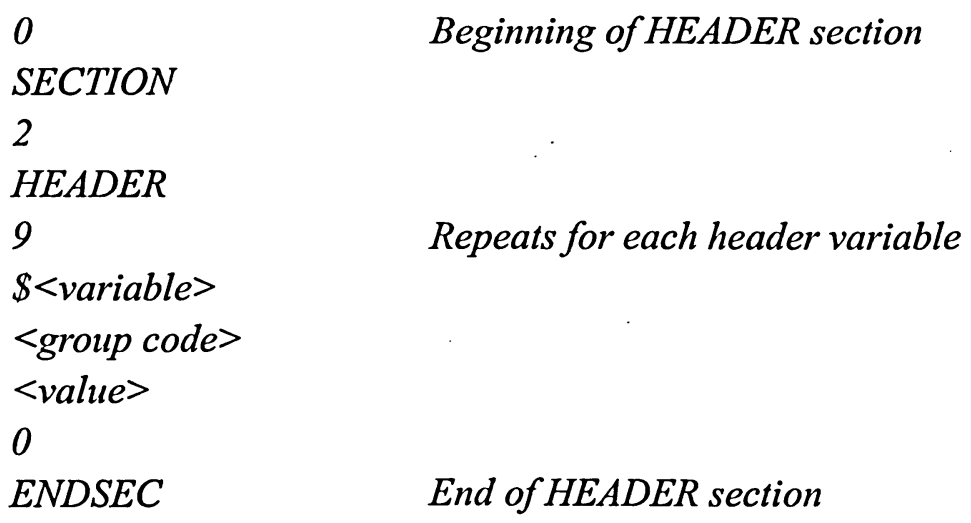

CLASSES section. Holds the information for application-defined classes, whose instances appear in the BLOCKS, ENTITIES, and OBJECTS sections of the database. A class definition is permanently fixed in class hierarchy. The following 
is an example of the CLASSES section of a DXF file:

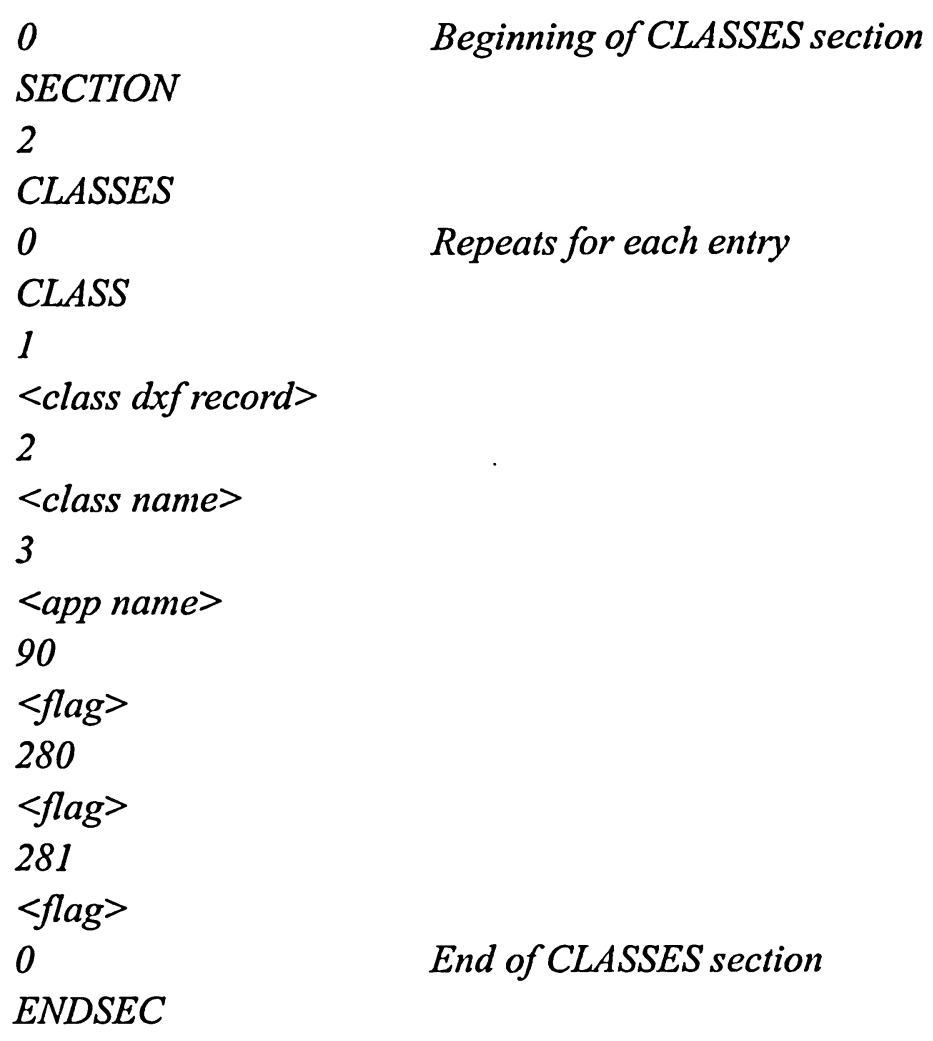

ENDSEC

TABLES section. Contains definitions for the following symbol tables:

APPID (application identification table)

BLOCK_RECORD (block reference table)

DIMSTYLE (dimension style table)

LAYER (layer table)

LTYPE (line type table)

STYLE (text style table)

UCS (user coordinate system table)

VIEW (view table) 
ASCII DXF Files | 165

VPORT (view-port configuration table)

BLOCKS section. Contains block definition and drawing entities that make up each block reference in the drawing.

ENTITIES section. Contains the graphical objects (entities) in the drawing, including block references (insert entities). The following is an example of the ENTITIES section of a DXF file:

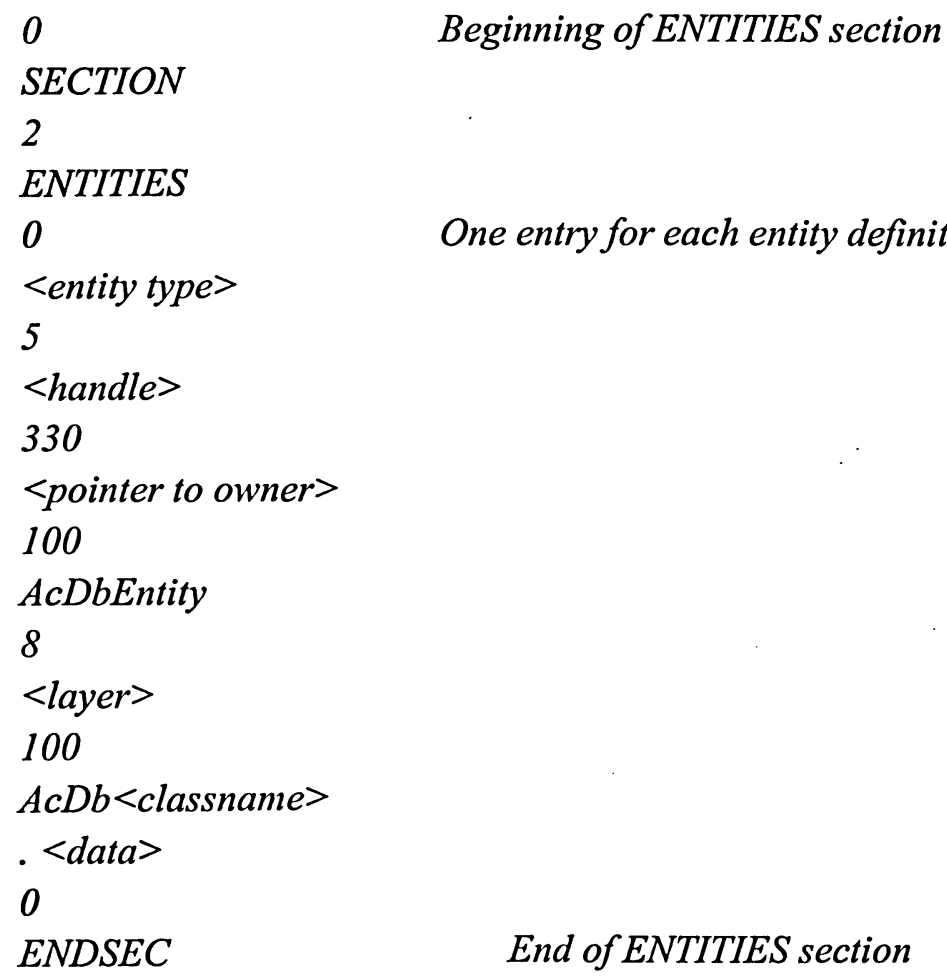

OBJECTS section. Contains the nongraphical objects in the drawing. All objects that are not entities or symbol table records or symbol tables are stored in this 
section. Examples of entries in the OBJECTS section are dictionaries that contain line styles and groups.

THUMBNAILIMAGE section. Contains the preview image data for the drawing. This section is optional.

The first group of data that we want to determine is the range of GIS data. This group is stored in HEADER section with name of "\$EXTMIN" and "\$EXTMAX". The part of this section is shown as below format.

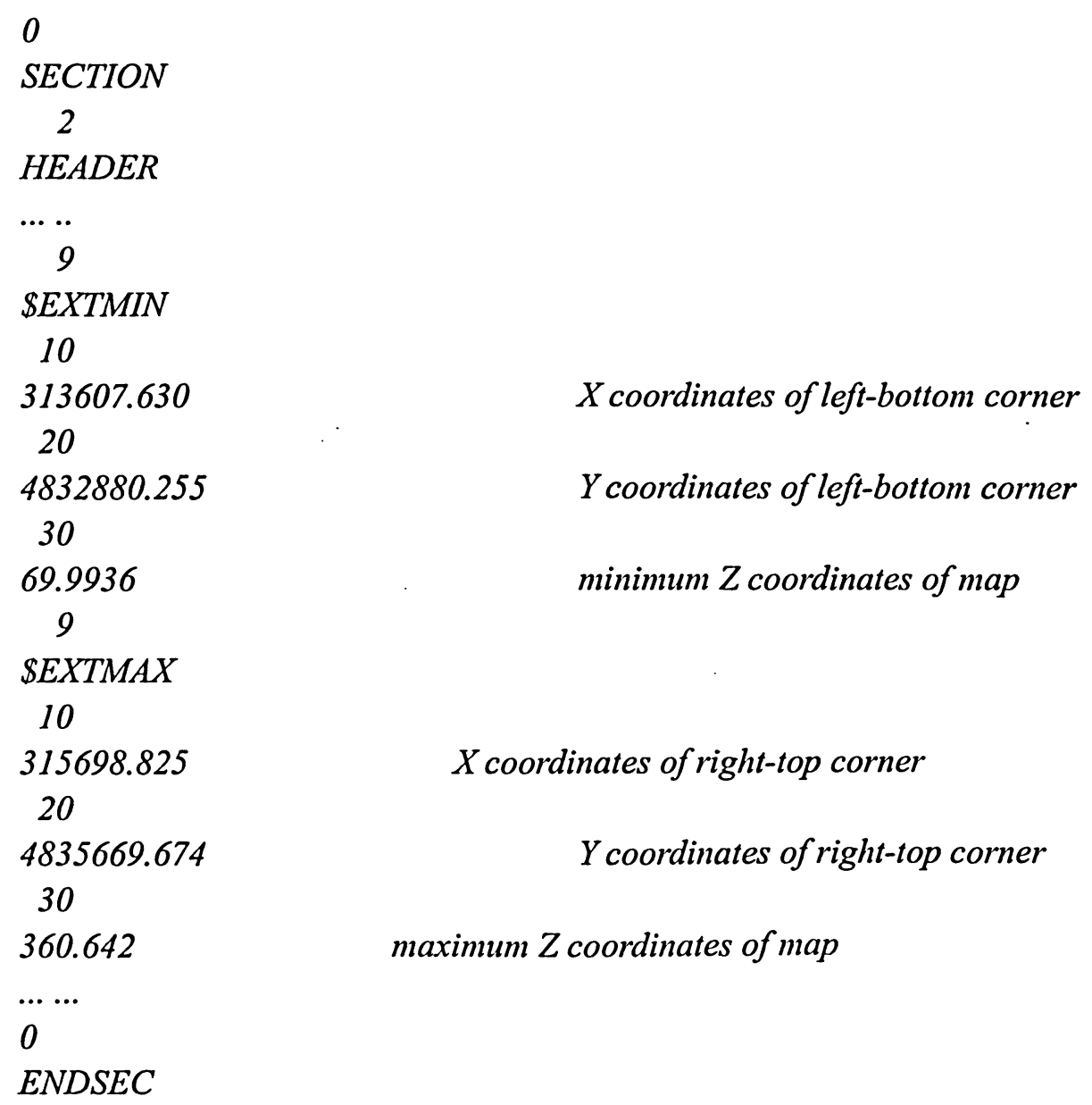


After determining the range of map, the properties and coordinates of utilities should be extracted from the DXF file. All these data are stored in ENTITIES section. For example, information of a manhole is saved in DXF file with following format.

$\begin{array}{ll}\quad 0 & \text { Class marker } \\ \text { INSERT } & \\ 5 & \text { Entity ID } \\ \text { C1D } & \\ 330 & \\ 11 A D B & \\ 100 & \text { Is Entity } \\ \text { AcDbEntity } & \\ 8 & \text { Property } \\ \text { ROUND_MANHOLE } & \\ 100 & \text { Subclass marker } \\ \text { AcDbBlockReference } & \\ 2 & \text { Block name } \\ 44 & \\ 10 & \text { X coordinates of insert point } \\ 314613.946 & \\ 20 & \text { Ycoordinates of insert point } \\ 4833499.7061 & \\ 30 & \text { Z coordinates of insert point } \\ 76.254 & \\ 41 & 41,42,43 \text { are not used in } \\ \text { transformation } & \\ 0.999990686758433 & \\ 42 & \\ 0.999990686758433 & \\ 43 & \\ 0.999990686758433 & \\ & \end{array}$

After needed the required information is extracted and saved in an ASCII file with following format.

minimum $\mathrm{X}$ coordinates maximum $\mathrm{X}$ coordinate 


\author{
minimum $\mathrm{Y}$ coordinates maximum $\mathrm{Y}$ coordinate \\ minimum $\mathrm{Z}$ coordinates maximum $\mathrm{Z}$ coordinate \\ total number of entities \\ entity property number of points of Entity \\ ...X Y Z value of each point \\ appendix value(optional)
}

\title{
3.2.3.2 GPS Navigation
}

Most GPS receivers support a navigation function in their specialized software programs. However, vendors do not supply users with an interface to use GPS receivers in their own programs. A GARMIN GPS10 was selected because it is a bluetooth-enabled wireless GPS receiver and inexpensive. When it is working in the National Marine Electronics Association (NMEA) mode, this kind of GPS receiver can send latitude, longitude coordinates and other information as an ASCII sentence to a target computer through a virtual serial communication port (performed by bluetooth technology). NMEA is a standard protocol, used by GPS receivers to transmit data. NMEA output is RS-232 compatible and uses 4800 bps, 8 data bits, no parity and one stop bit. NMEA sentences are all ASCII. Each sentence begins with a dollar sign (\$) and ends with a carriage return linefeed character sequence. The following NMEA sentence describes the position information of the current GPS receiver. 
"\$GPGGA, hhmmss.ss, 111l.11, a, yyyyy.yy, a, x, xx, x.x, x.x, M, x.x, M, x.x, xxxx*hh"

where:

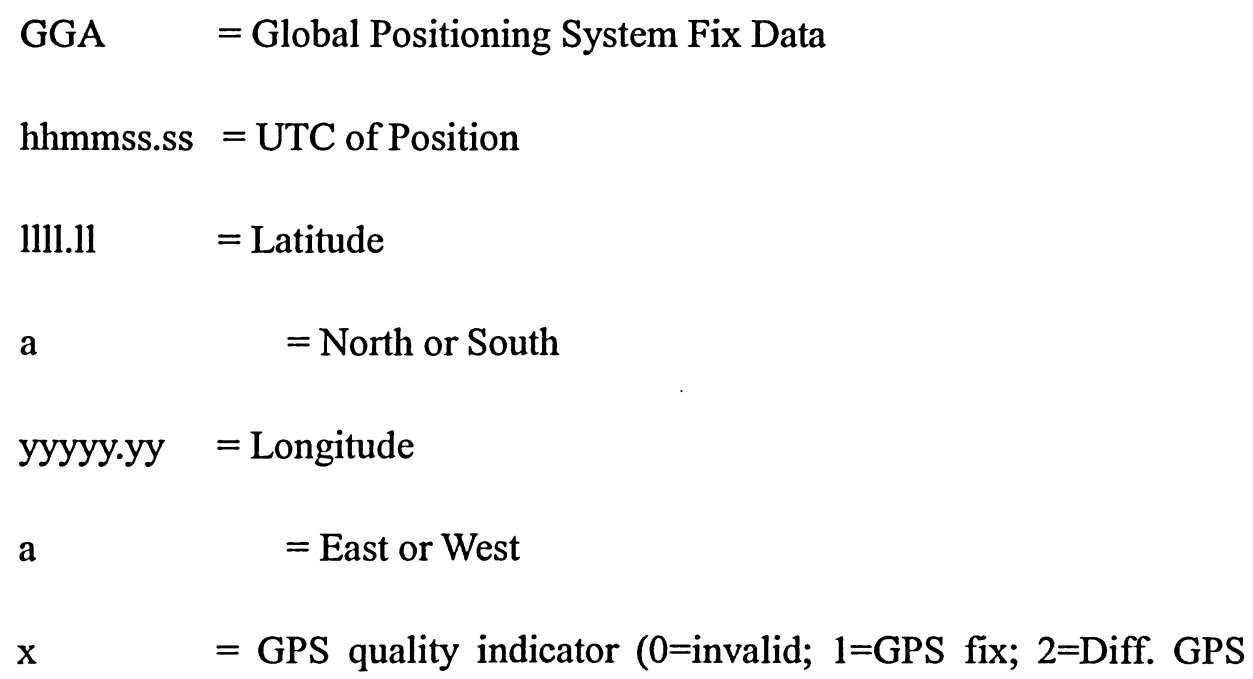

fix)

$\mathrm{xx} \quad=$ Number of satellites in use [not those in view]

$\mathrm{x} . \mathrm{x}=$ Horizontal dilution of position

$\mathrm{x} . \mathrm{x}=$ Antenna altitude above/below mean sea level (geoid)

$\mathrm{M} \quad=$ Metres (Antenna height unit)

X.X = Geoidal separation (Diff. between WGS-84 earth ellipsoid and mean sea level.)

$\mathrm{M} \quad=$ Metres (Units of geoidal separation)

$\mathrm{x} . \mathrm{x}=$ Age in seconds since last update from diff. reference station

$\operatorname{xxxx} \quad=$ Diff. reference station ID\#

*hh $\quad=$ the Checksum data 
Since GPS10 supports geodetic coordinates where the coordinates of GIS dataset is the Modified Transverse Mercator (MTM) projection (The scale factor for this projection is 0.9999 and the false easting is 304800.0 metres.), we must transform geodetic coordinates of GPS position to the MTM coordinates system when working on this project. After this transformation, GPS receiver can be used for navigating in the GIS data set to complete GCPs collection.

\subsubsection{GCPs Collection}

The GIS data set is scaled to suit the site scale then the user navigates to the target area by using the GPS receiver. The program will display all needed vertices on the computer screen. All vertices have 3D coordinates and can be used as GCPs. Each GCP has the information of point name, $\mathrm{X}, \mathrm{Y}$ and $\mathrm{Z}$ coordinates. After moving the mouse cursor over a vertex, the coordinates of that vertex can be obtained and saved into a GCP file. This file then can be directly used in the external orientation program.

\subsection{D Reconstruction}

\subsubsection{Introduction}

Here, the expression 3D reconstruction means using photogrammetric methods to obtain 3D coordinates of target point and to save them with special features. There are 
two main photogrammetric methods that can be used in computing 3D coordinates, space intersection and bundle adjustment. Space intersection is used when only two images are employed. This method does not need good initial approximations. But errors cannot be corrected through this method. Bundle adjustment uses the collinearity equations to calculate 3D coordinates of ground point. It is used when more than three images overlap the target area. However, collinearity equations are nonlinear and good initial approximations are needed. The current solution uses space intersection to calculate space coordinates as the first step; if 3 or more images overlap target area, using the results of the space intersection as initial approximations and using the bundle adjustment to correct them. The methodologies of space intersection and bundle adjustment are described in Sections 3.3.2 and 3.2.3.

Whether space intersection or bundle adjustment is used, the image coordinates of homologous points are required. Generally, homologous points are not easy to measure in large-scale images used in our project, as most target points do not have point features. Epipolar geometry is normally used in stereo vision to solve the correspondence problem in two images, i.e. for a given 2D point in the first image, it would be found the corresponding 2D point in the second one. Section 3.3.4 describes the methodologies of epipolar geometry. 


\subsubsection{Space Intersection}

If two images are employed in calculating these coordinates, this procedure is known as space intersection, so called because corresponding rays to the same object point from the two images must intersect at the point, as shown in Figure 3-5. Here, $\left(X_{1}, Y_{1}, Z_{1}\right)^{T}$ and $\left(X_{2}, Y_{2}, Z_{2}\right)^{T}$ are ground coordinates of homologous points in first and second image. Equation (3-20) shows how to compute these coordinates. $\left(X_{A}, Y_{A}, Z_{A}\right)^{T}$ are the coordinates of ground point. The computation of $\left(X_{A}, Y_{A}, Z_{A}\right)^{T}$ is described as follows.

$$
\left(\begin{array}{c}
X_{1} \\
Y_{1} \\
Z_{1}
\end{array}\right)=R_{1}\left(\begin{array}{c}
x_{1} \\
y_{1} \\
-f
\end{array}\right) \text { and }\left(\begin{array}{c}
X_{2} \\
Y_{2} \\
Z_{2}
\end{array}\right)=R_{2}\left(\begin{array}{c}
x_{2} \\
y_{2} \\
-f
\end{array}\right)
$$

where:

$$
\begin{array}{ll}
x_{1}, y_{1}: & \text { image coordinates of first image } \\
x_{2}, y_{2}: & \text { image coordinates of second image } \\
R_{1}: & \text { rotation matrix of first image } \\
R_{2}: & \text { rotation matrix of second image } \\
f: & \text { the focal length of a digital camera }
\end{array}
$$




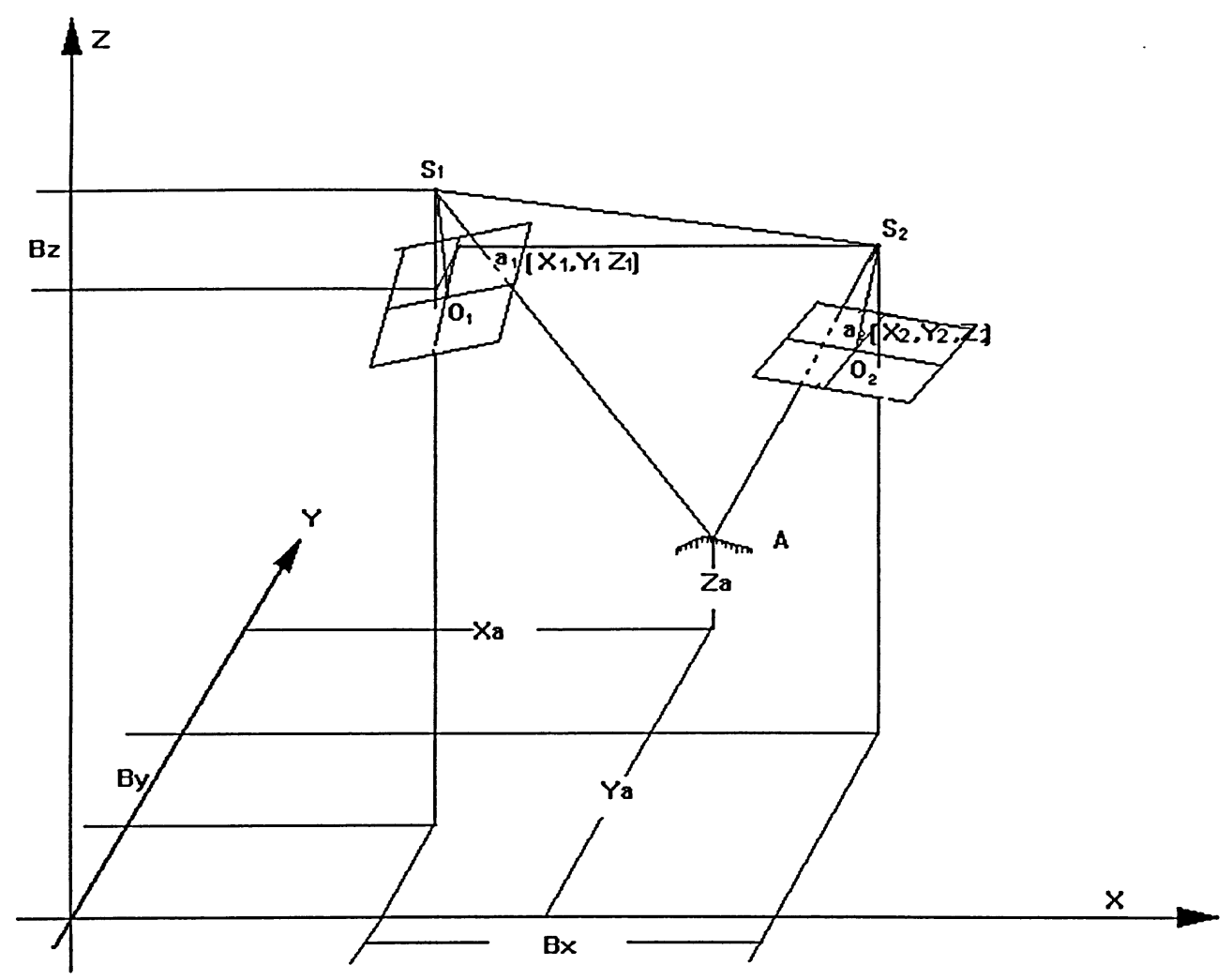

Figure 3-5 Space intersection 1

Figure 3-6 is the result of projecting Figure 3-5 to the $X-Z$ plane. In Figure 3-6,

$$
\begin{aligned}
& D X_{1}=X_{A}-X_{S 1} \\
& D X_{2}=X_{A}-X_{S 2} \\
& D Z_{1}=Z_{A}-Z_{S 1} \\
& D Z_{2}=Z_{A}-Z_{S 2} \\
& B_{X}=X_{S 2}-X_{S 1} \\
& B_{Y}=Y_{S 2}-Y_{S 1} \\
& B_{Z}=Z_{S 2}-Z_{S 1}
\end{aligned}
$$




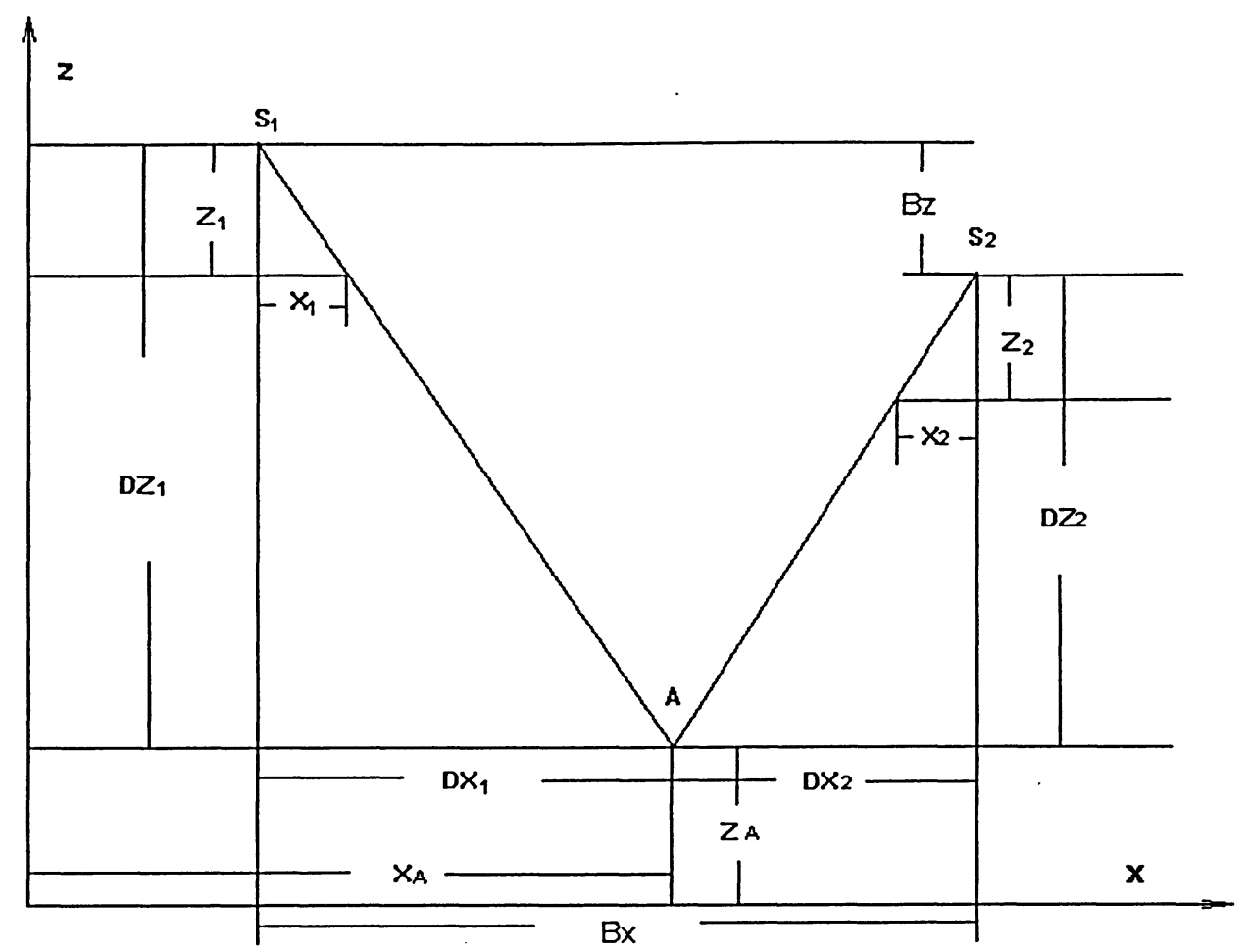

Figure 3-6 Space intersection 2

From Figure 3-6, we have:

$\frac{X_{1}}{D X_{1}}=\frac{Z_{1}}{D Z_{1}} \quad$ and $\quad \frac{X_{2}}{D X_{2}}=\frac{Z_{2}}{D Z_{2}}$

since

$D X_{1}=X_{A}-X_{S 1}, \quad D X_{2}=X_{A}-X_{S 2}$

$D Z_{1}=Z_{A}-Z_{S 1}, \quad D Z_{2}=Z_{A}-Z_{S 2}$

$B_{X}=X_{s 2}-X_{s 1}, \quad B_{Z}=Z_{s 2}-Z_{s 1}$

Equations (3-22) can be transformed to Equations (3-23)

$\frac{X_{1}}{B_{X}+D X_{2}}=\frac{Z_{1}}{B_{Z}+D Z_{2}}(A)$ and $\frac{X_{2}}{D X_{2}}=\frac{Z_{2}}{D Z_{2}}(B)$ 
(B) can be transformed to (C)

$$
D X_{2}=\frac{X_{2}}{Z_{2}} D Z_{2}
$$

Substitute (C) into (A), we obtain

$D Z_{2}\left(X_{1} Z_{2}-X_{2} Z_{1}\right)=\left(Z_{1} B_{X}-X_{1} B_{Z}\right) Z_{2}$

Then

$D Z_{2}=\frac{Z_{1} B_{X}-X_{1} B_{Z}}{X_{1} Z_{2}-X_{2} Z_{1}} Z_{2}$

and

$Z_{A}=D Z_{2}+Z_{S 2}$

Based on the same method, we obtain

$$
\begin{aligned}
& D X_{2}=\frac{Z_{1} B_{X}-X_{1} B_{Z}}{X_{1} Z_{2}-X_{2} Z_{1}} X_{2}, D Y_{2}=\frac{Z_{1} B_{X}-X_{1} B_{Z}}{X_{1} Z_{2}-X_{2} Z_{1}} Y_{2} \quad, D Z_{2}=\frac{Z_{1} B_{X}-X_{1} B_{Z}}{X_{1} Z_{2}-X_{2} Z_{1}} Z_{2} \\
& X_{A}=D X_{2}+X_{S 2}, \quad Y_{A}=D Y_{2}+Y_{S 2} \quad, \quad Z_{A}=D Z_{2}+Z_{S 2}
\end{aligned}
$$

From this method we can see that if there are errors in exterior elements or interior elements, a wrong result will be generated as it is derived directly from such elements. A solution that can be used to correct such problems is a bundle adjustment.

\subsubsection{Bundle Adjustment}

As mentioned in Section 3.2.1, Equation (3-1) can be used for generating 3D coordinates of target point if more than two digital images overlap target area and the 
interior elements, distortion coefficients and exterior elements are known. As mentioned in Section 3.2, Equation (3-1) is nonlinear and can be rewritten as Equation (3-15). According to Taylor's series, Equation (3-15) can be expressed in a linearized form by taking partial derivatives with respect to the ground coordinates:

$$
\begin{aligned}
& F_{0}+\left(\frac{\partial F}{\partial X}\right) d X_{A}+\left(\frac{\partial F}{\partial Y}\right) d Y_{A}+\left(\frac{\partial F}{\partial Z}\right) d Z_{A}=x \\
& G_{0}+\left(\frac{\partial G}{\partial X}\right) d X_{A}+\left(\frac{\partial G}{\partial Y}\right) d Y_{A}+\left(\frac{\partial G}{\partial Z}\right) d Z_{A}=y
\end{aligned}
$$

In Equation (3-25), $F_{0}$ and $G_{0}$ are functions $\mathrm{F}$ and $\mathrm{G}$ of Equation (3-15) evaluated at the initial approximations for the three unknown ground coordinates; the terms $\left(\frac{\partial F}{\partial X_{A}}\right),\left(\frac{\partial F}{\partial Y_{A}}\right),\left(\frac{\partial G}{\partial Z_{A}}\right)$, are partial derivatives of functions $\mathrm{F}$ and $\mathrm{G}$ with respect to the indicated unknown $\left(X_{A}, Y_{A}, Z_{A}\right)^{T}$ evaluated at the initial approximations; and $d X_{A}, d Y_{A}, d Z_{A}$, are unknown corrections to be applied to the initial approximations. The initial approximations can be directly from space intersection. Equation (3-25) can be simplified as following forms:

$$
\begin{aligned}
& b_{14} d X_{A}+b_{15} d Y_{A}+b_{16} d Z_{A}=J+v_{x} \\
& b_{24} d X_{A}+b_{25} d Y_{A}+b_{26} d Z_{A}=K+v_{y}
\end{aligned}
$$

where 


$$
\begin{aligned}
& b_{14}=\frac{f}{q^{2}}\left(r_{31} R-r_{11} Q\right) \\
& b_{15}=\frac{f}{q^{2}}\left(r_{32} R-r_{12} Q\right) \\
& b_{16}=\frac{f}{q^{2}}\left(r_{33} R-r_{13} Q\right) \\
& J=x-x_{0}-\Delta x+f \frac{R}{Q} \\
& b_{24}=\frac{f}{q^{2}}\left(r_{31} S-r_{21} Q\right) \\
& b_{25}=\frac{f}{q^{2}}\left(r_{32} S-r_{22} Q\right) \\
& b_{26}=\frac{f}{q^{2}}\left(r_{33} S-r_{23} Q\right) \\
& K=y-y_{0}-\Delta y+f \frac{S}{Q}
\end{aligned}
$$

This method is called a bundle adjustment where collinearity equations based on light ray bundles are employed. As three or more images are used for calculating space coordinates, which means there are six or more conditions to calculate 3 unknowns, the errors can be adjusted using the least square adjustment method.

Considering the exterior elements contain errors, these elements also can be corrected using a bundle adjustment. Equation (3-1) can be rewritten in a linearize form as follows: 


$$
\begin{aligned}
& F_{0}+\left(\frac{\partial F}{\partial \omega}\right) d \omega+\left(\frac{\partial F}{\partial \phi}\right) d \phi+\left(\frac{\partial F}{\partial \kappa}\right) d \kappa+\left(\frac{\partial F}{\partial X_{S}}\right) d X_{S}+\left(\frac{\partial F}{\partial Y_{S}}\right) d Y_{S}+\left(\frac{\partial F}{\partial Z_{S}}\right) d Z_{S} \\
& +\left(\frac{\partial F}{\partial X}\right) d X_{A}+\left(\frac{\partial F}{\partial Y}\right) d Y_{A}+\left(\frac{\partial F}{\partial Z}\right) d Z_{A}=x \\
& G_{0}+\left(\frac{\partial G}{\partial \omega}\right) d \omega+\left(\frac{\partial G}{\partial \phi}\right) d \phi+\left(\frac{\partial G}{\partial \kappa}\right) d \kappa+\left(\frac{\partial G}{\partial X_{S}}\right) d X_{S}+\left(\frac{\partial G}{\partial Y_{S}}\right) d Y_{S}+\left(\frac{\partial G}{\partial Z_{S}}\right) d Z_{S} \\
& +\left(\frac{\partial G}{\partial X}\right) d X_{A}+\left(\frac{\partial G}{\partial Y}\right) d Y_{A}+\left(\frac{\partial G}{\partial Z}\right) d Z_{A}=y
\end{aligned}
$$

Equation (3-25) can be simplified to the following forms:

$$
\begin{aligned}
& b_{11} d \omega+b_{12} d \phi+b_{13} d \kappa-b_{14} d X_{S}-b_{15} d Y_{S}-b_{16} d Z_{S}+ \\
& b_{14} d X_{A}+b_{15} d Y_{A}+b_{16} d Z_{A}=J+v_{x} \\
& b_{21} d \omega+b_{22} d \phi+b_{23} d \kappa-b_{24} d X_{S}-b_{25} d Y_{S}-b_{26} d Z_{S}+ \\
& b_{24} d X_{A}+b_{25} d Y_{A}+b_{26} d Z_{A}=K+v_{y}
\end{aligned}
$$

where $b_{i j}$ can be computed using the same method as shown before. The initial approximations can be directly obtained from the external orientation and space intersection results.

\subsubsection{Epipolar Geometry}

Epipolar geometry expresses the geometric relationship between two images of a rigid body. Two perspective images of a single rigid object scene are related by the so-called epipolar geometry, which can be described by a $3 \times 3$ matrix. If the interior elements of the images (e.g., the focal length, the coordinates of the principal point, etc.) are known, the normalized image coordinates can be used, and the matrix is known as the essential matrix. Otherwise, the pixel image coordinates are used, and 
the matrix is known as the fundamental matrix. It contains all geometric information that is necessary for establishing correspondences between two images, from which 3D structure of the perceived scene can be inferred.

\subsubsection{What is Epipolar Geometry?}

The epipolar geometry between two views is essentially the geometry of the intersection of the image planes with the pencil of planes having the baseline as axis (the baseline is the line joining the camera centres). This geometry is usually motivated when considering the search for corresponding points in stereo matching, and we will start from that objective here. Suppose a point $\mathbf{X}$ in 3-space is imaged in two views, at point $\mathbf{x}$ in the first image, and $\mathbf{x}^{\prime}$ in the second image. As shown in Figure 3-7, the image points $\mathbf{x}$ and $\mathbf{x}$ ', space point $\mathbf{X}$, and camera centres are coplanar where this plane is denoted as $\pi$. Clearly, the rays back-projected from $\mathbf{x}$ and $\mathbf{x}$ ' intersect at $\mathbf{X}$, and the rays are coplanar, lying in $\pi$. It is this latter property that is of most significance in searching for a correspondence.

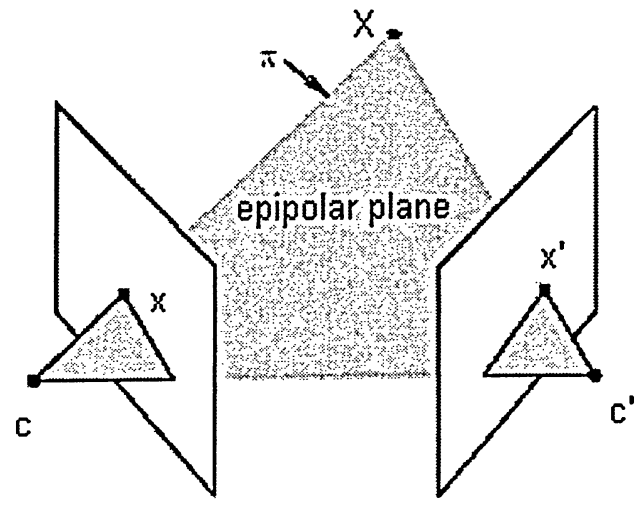

:i

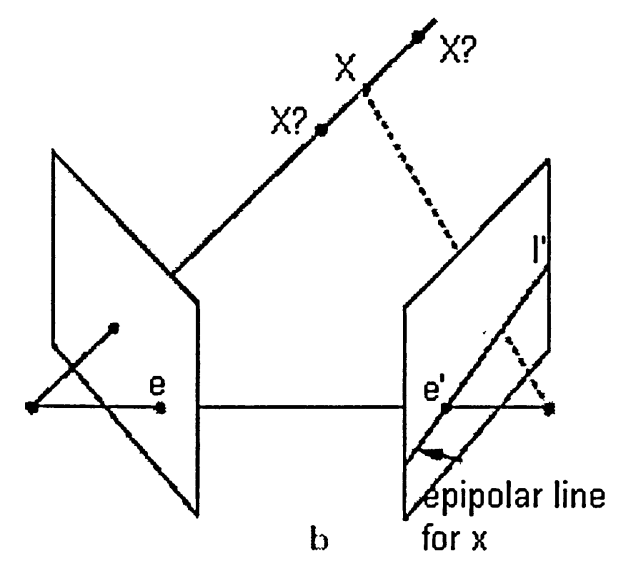

Figure 3-7 Point correspondence geometry 
Supposing now that only $\mathbf{x}$ is known, how the corresponding point $\mathbf{x}$ ' is constrained. The plane $\pi$ is determined by the baseline and the ray defined by $\mathbf{x}$. From the above, the ray corresponding to the (unknown) point $x^{\prime}$ lies in $\pi$. Hence, the point $x^{\prime}$ lies on the line of intersection l' of $\pi$ with the second image plane. This line l' is the image in the second view of the ray back-projected from $\mathbf{x}$. In terms of a stereo correspondence algorithm, the benefit is that the search for the point corresponding to $\mathrm{x}$ need not cover the entire image plane but can be restricted to the line l'.

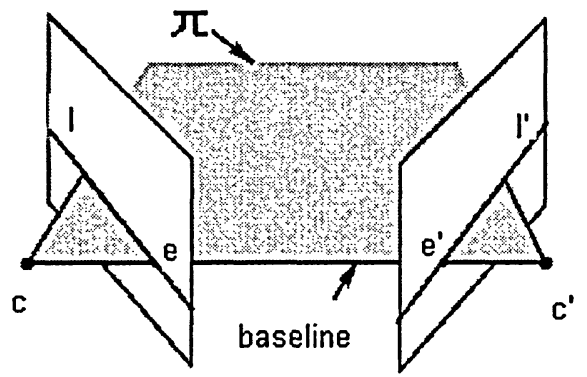

a

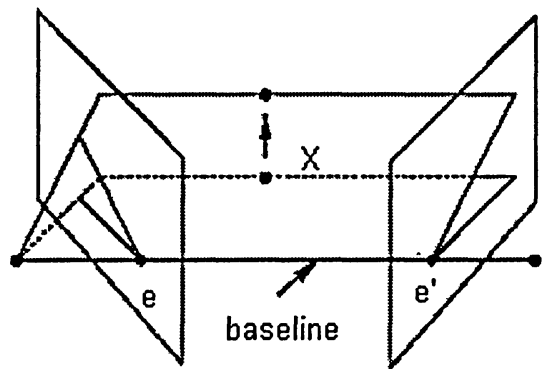

b

Figure 3-8 Epipolar geometry

The geometric entities involved in epipolar geometry are illustrated in Figure 3-8. The terminology is:

The epipole is the point of intersection of the line joining the camera centres (the baseline) with the image plane. In other words, the epipole is the image in one view of the camera centre of the other view. It is also the vanishing point of the baseline (translation) direction.

An epipolar plane is a plane containing the baseline. There is a one-parameter 
family (a pencil) of epipolar planes.

An epipolar line is the intersection of an epipolar plane with the image plane. All epipolar lines intersect at the epipole. An epipolar plane intersects the left and right image planes in epipolar lines, and defines the correspondence between the lines.

\subsubsection{The Fundamental Matrix F}

The fundamental matrix is the algebraic representation of epipolar geometry. It satisfies:

(1) $\mathrm{F}$ is a rank 2 homogeneous matrix with 7 degrees of freedom,

(2) If $\mathbf{x}$ and $\mathbf{x}^{\prime}$ are homologous points, then $x^{\prime T} F x=0$,

(3) $l^{\prime}=F x$ is the epipolar line corresponding to $\mathbf{x}$,

(4) $l=F^{T} x^{\prime}$ is the epipolar line corresponding to $\mathbf{x}^{\prime}$ and

(5) Epipoles satisfy $F e=0$ and $F^{T} e^{\prime}=0$.

The fundamental matrix can be computed from camera projection matrices $\mathrm{P}, \mathrm{P}$ '. The ray back-projected from $\mathbf{x}$ by $\mathrm{P}$ is obtained by solving $\mathbf{x}=\mathrm{PX}$. Or

$$
\left(\begin{array}{l}
x \\
y \\
1
\end{array}\right)=P\left(\begin{array}{l}
X \\
Y \\
Z \\
1
\end{array}\right)
$$

where $\mathbf{P}$ is a $3 \times 4$ matrix. Equation (3-29) can be rewritten to Equation (3-30)

$$
\mathbf{X}(\lambda)=\mathrm{P}^{+} \mathbf{x}+\lambda \mathbf{C}
$$

where $\mathrm{P}^{+}$is the pseudo-inverse of $\mathrm{P}$, i.e. $\mathrm{P} \mathrm{P}^{+}=\mathrm{I}$, and $\mathrm{C}$ its null-vector, namely the 
camera centre, defined by $\mathrm{PC}=\mathbf{0}$. The ray is parametrized by the scalar $\lambda$. In particular, two points on the ray are $\mathrm{P}^{+} \mathbf{x}($ at $\lambda=0$ ), and the first camera centre $\mathbf{C}$ (at $\lambda$ $=\infty)$. These two points are projected by the second camera $\mathrm{P}^{\prime}$ at $\mathrm{P}^{\prime} \mathrm{P}^{+} \mathbf{x}$ and $\mathrm{P}^{\prime} \mathrm{C}$, respectively, in the second view. The epipolar line is the line joining these two projected points, namely $\mathrm{l}^{\prime}=\left(\mathrm{P}^{\prime} \mathrm{C}\right) \times\left(\mathrm{P}^{\prime} \mathrm{P}^{+} \mathrm{x}\right)$. The point $\mathrm{P}^{\prime} \mathrm{C}$ is the epipole in the second image, namely the projection of the first camera centre, and may be denoted by e'. Thus,

$$
l^{\prime}=e^{\prime} \times\left(P^{+}\right) x=F x
$$

where the fundamental matrix $\mathrm{F}$ can be calculated as

$$
\mathrm{F}=\mathrm{e}^{\prime} \times\left(\mathrm{PP}^{+}\right)
$$

When a target point in the first image is measured, the corresponding epipolar line can be generated and drawn in the second image since digital images are employed in our project. It will help operators easily find corresponding point in the second image. Furthermore, if three images overlap target area and the image positions of the first two images are measured, two epipolar lines can be computed and intersect in the third image. The point of intersection gives the initial position of target point in the third image.

\subsection{Chapter Summary}

This chapter describes the processing steps and their methodologies of this procedure. 
In photogrammetry, the interior elements that include focal length and the position of principal point should be computed through camera calibration before the camera is used. The distortion coefficients of lens are also calculated through this procedure. In order to make the processing easier and achieve a higher accuracy result, image matching and LSM are involved.

External orientation is a procedure to determine the position and orientation parameters called exterior elements. Without external orientation, the space coordinates cannot be calculated. GCPs have important rolls in external orientation. An impact method is presented in this chapter that can easily obtain GCPs used in external orientation to calculate exterior elements.

After both interior and exterior elements are known, space coordinates of target point can be computed using space intersection if two images overlap target area. Furthermore, if three images overlap the target area, a bundle adjustment can be employed for correcting space coordinates and adjusting any errors. Epipolar geometry is also introduced in this chapter. The fundamental matrix is used in calculating epipolar lines that can help operators easily measure image coordinates of target point in digital images. 


\section{CASE STUDY OF CAMERA CALIBRATION}

This designated study involved building two software programs to complete camera calibration. The objective of the first program is to automatically detect image coordinates of GCPs that can be used for calibrating our digital camera. The second program is used for camera calibration. The distribution of errors and distortions are shown in this program. The corresponding results obtained from the analysis are presented in Section 4.2 .2 of this chapter.

\subsection{Automatic Detection Program}

This program is a data preparation program used for camera calibration. The objective of this program is to automatically detect image coordinates of GCPs called reference points in this chapter. This program is a Microsoft Windows program coded by Visual $\mathrm{C}++$. Figure 4-1 shows the interface of this program.

After loading the digital image, the program can automatically detect image coordinates of GCPs. This procedure can be divided into three processing steps. The first step is to automatically detect the initial positions of reference points in digital image using image matching technology. The second step is obtaining the accurate positions of reference points using LSM. The third step is merging such reference points and corresponding GCPs together in order that they can be used for camera 
calibration.

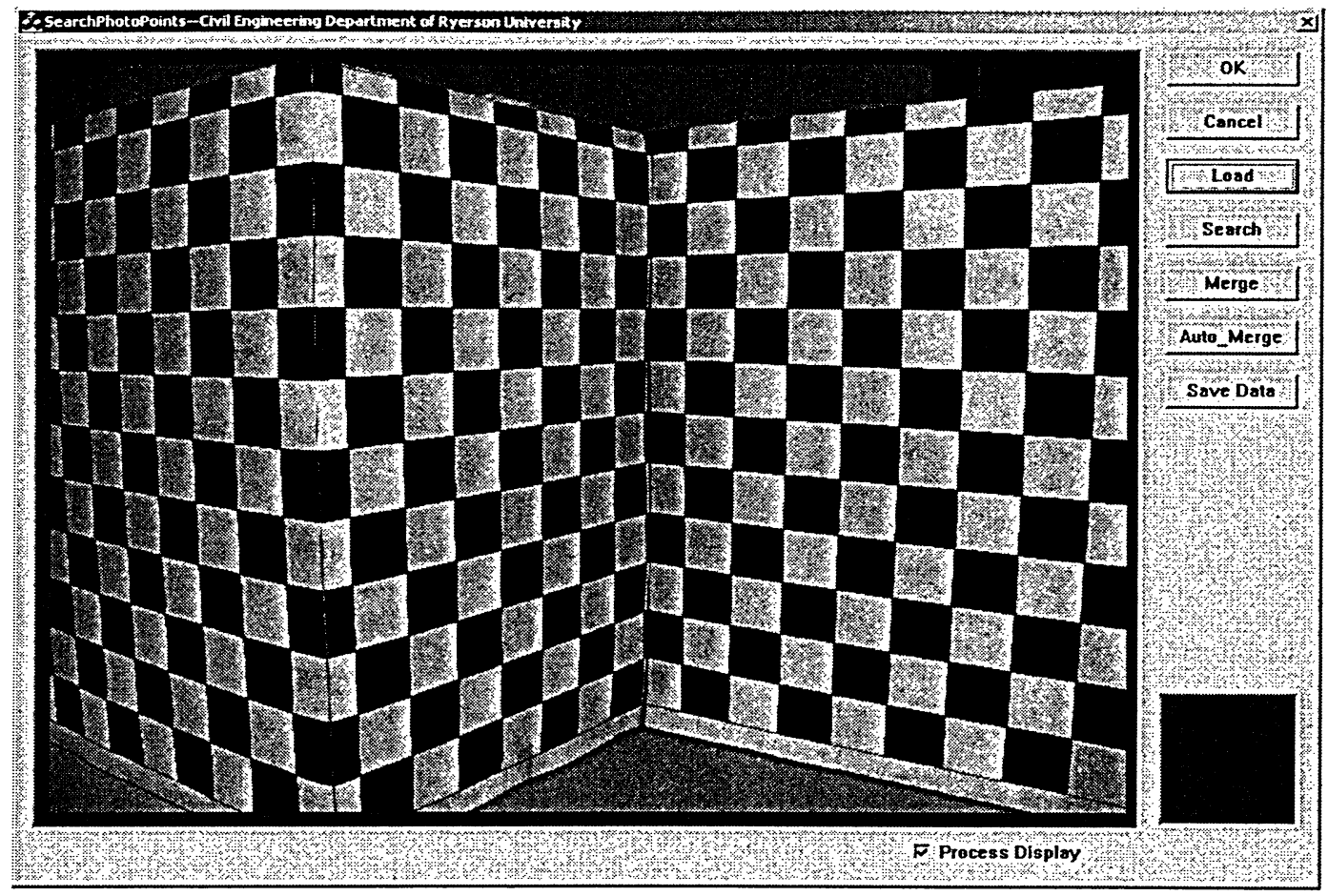

Figure 4-1 The interface of automatic detection program

\subsubsection{The Detection of Reference Points}

As mentioned in Section 3.1.3, based on the control pattern, two kinds of images are employed as fiducial images in order to detect the reference points. And, an image window called matching window is used for extracting grey values from original image at the start position (left-bottom corner). If the current point in the original image is checked as a reference point, it will be added to the reference point table.

The start position is changed pixel by pixel and the above steps are repeated until the matching window reaches the top-right corner of the original image. Then initial positions of all reference points are, thus, detected. Figure 4-2 illustrates the flow chart of this procedure. 


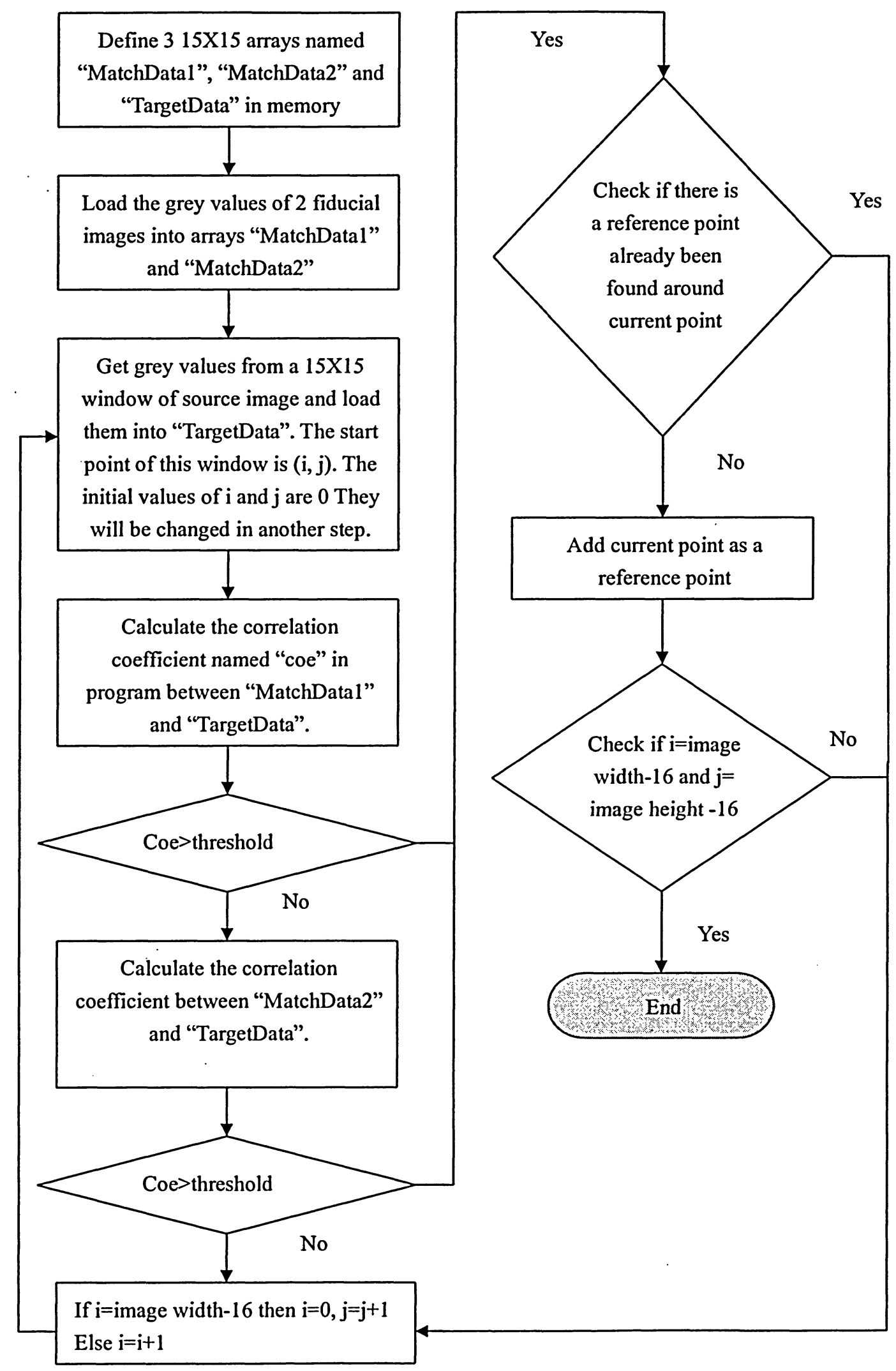

Figure 4-2 Flow chart of detecting initial positions of reference points 


\subsubsection{Obtaining Accurate Positions Using LSM}

After the initial positions of reference points are detected, the next step is obtaining the accurate positions using LSM. As mentioned in Section 3.1.3, LSM not only considers illumination and reflectance differences between the two images but also the geometric distortion of the regular image tessellation caused by unknown orientation parameters, tilted surface patch, a surface patch with relief, etc. The previous step gives the approximations within accuracy of a few pixels. These approximations can be used in LSM as initial values. Figure 4-3 illustrates the flow chart for obtaining accurate positions using LSM. Figure 4-4 shows an example result of automatically detected reference points.

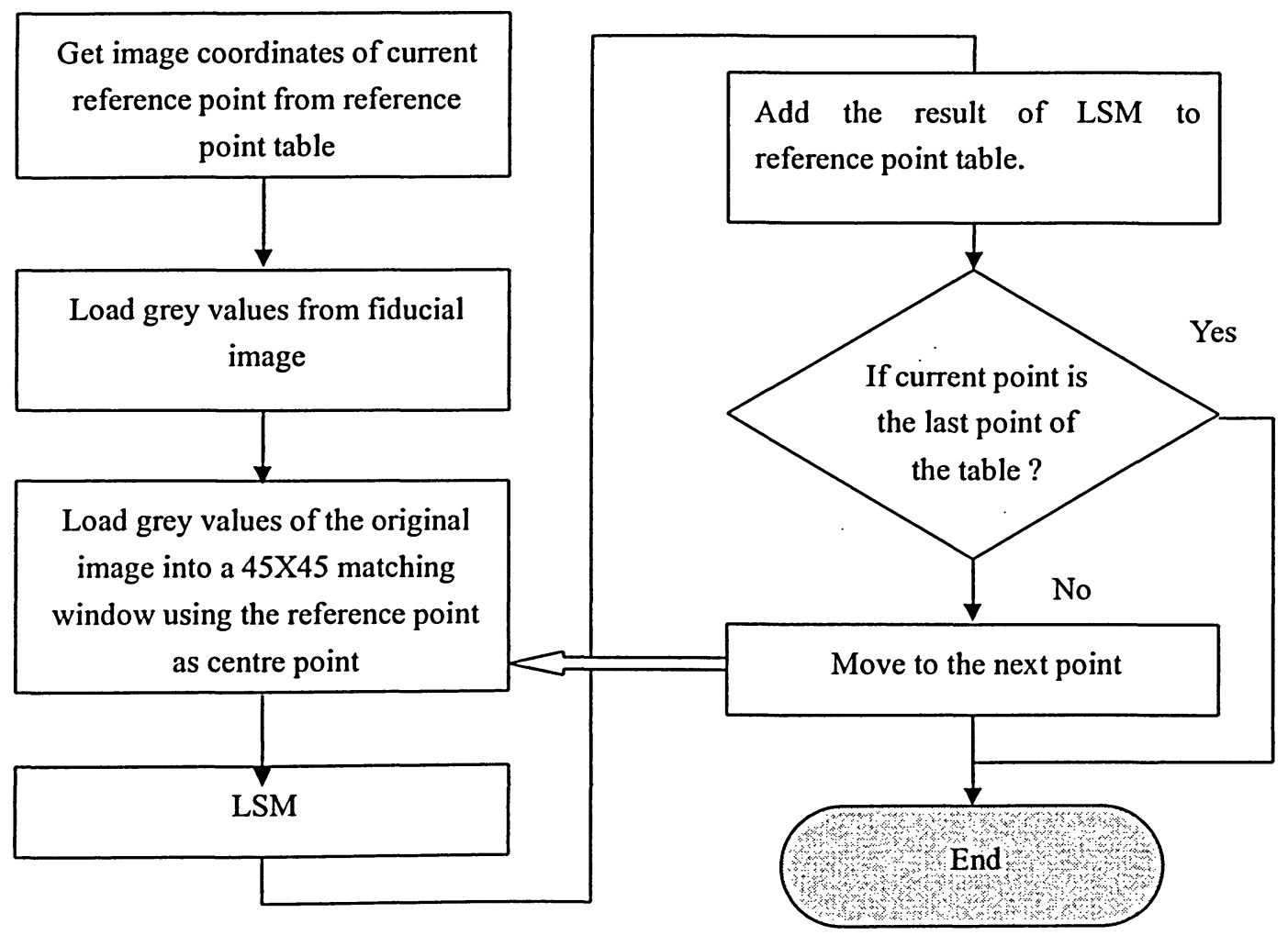

Figure 4-3 Flow chart of obtaining accurate positions using LSM. 


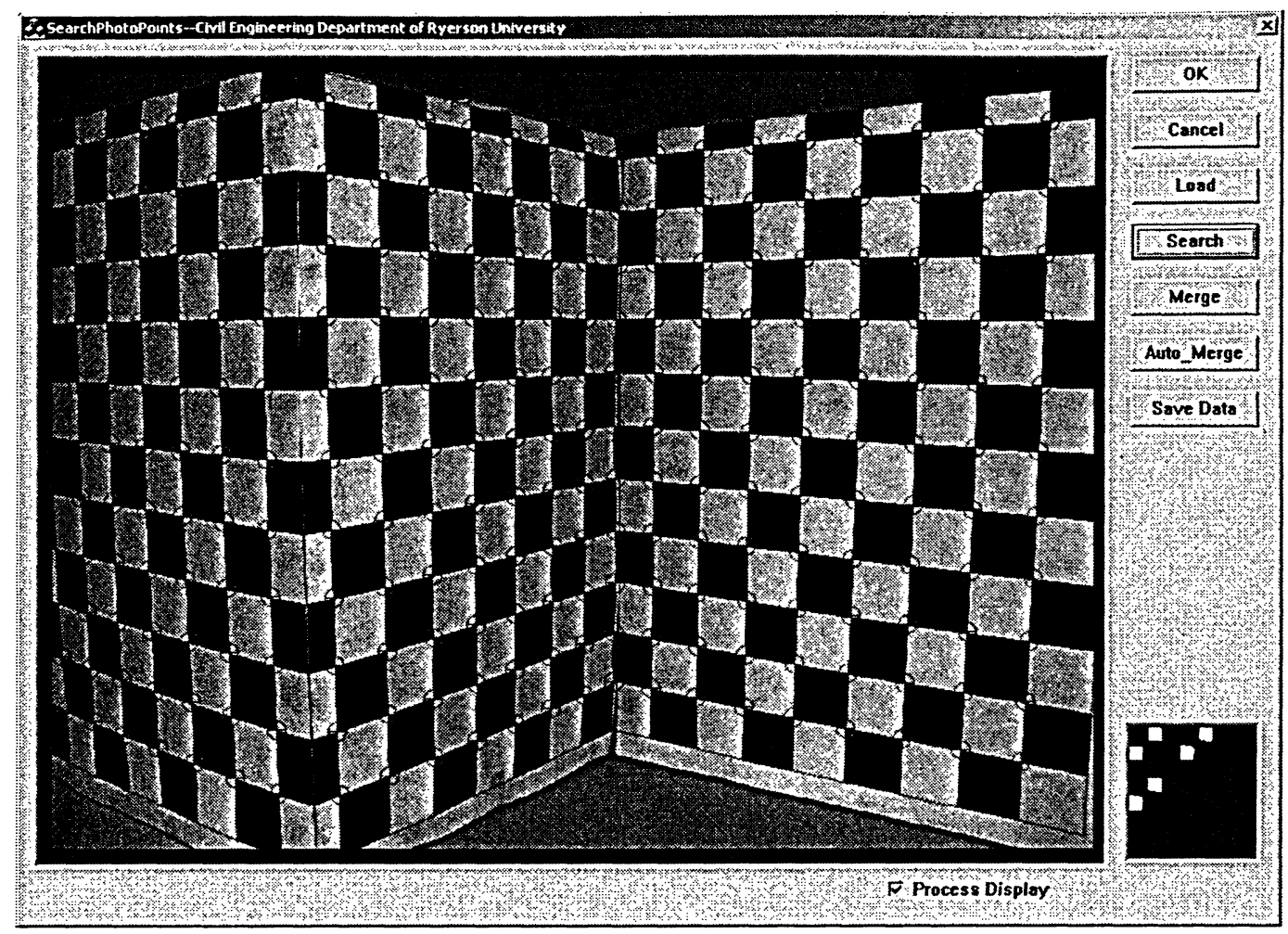

Figure 4-4 An example result of automatic detection of reference points

\subsubsection{The Combination of Reference Points and GCPs}

Although reference points are obtained through the above two steps, the relationships between them and GCPs are still unknown. Camera calibration cannot be processed without building up such relationships.

The DLT can be used for building up these relationships. If the relationships between at least 6 reference points and their corresponding GCPs that have a good distribution are known, a rough set of interior elements and exterior elements can be computed using the DLT method. Based on these data and letting $\Delta x=\Delta y=0$, approximate image coordinates of each GCP can be computed using Equation (3-1). The program 
then compares each pair of image coordinates to each reference point by calculating the distance between them, if the distance is less than 40 pixels, then current GCP corresponds to the current reference point. At least 8 pairs of reference points and GCPs are used in this program for a least-squares adjustment. As the rough set of interior and exterior elements of the camera is easy to calculate, the key problem that must be solved is how to determine the relationships between these reference points and their corresponding GCPs. There are two methods presented in this thesis and coded in the software program to solve this problem.

The first method is to directly determine corresponding points step by step. As shown in Figure 4-4, when a reference point is selected, the GCPs window will pop up as shown in Figure 4-5. The distribution of GCPs is displayed in this window. So the corresponding GCP of that selected reference point could be found and selected in this window. This procedure is repeated until all 8 pairs of reference points and corresponding GCPs are determined. Then the result can be used for computing the approximate parameters of interior and exterior elements and combining the reference points and corresponding GCPs together, respectively. The advantage of this method is that it works for most cases such as big angular orientations. The disadvantage of this method is that it requires a long time for operator to complete it and it is possible to make mistakes during this procedure as operator should choose target point from hundreds of points. 


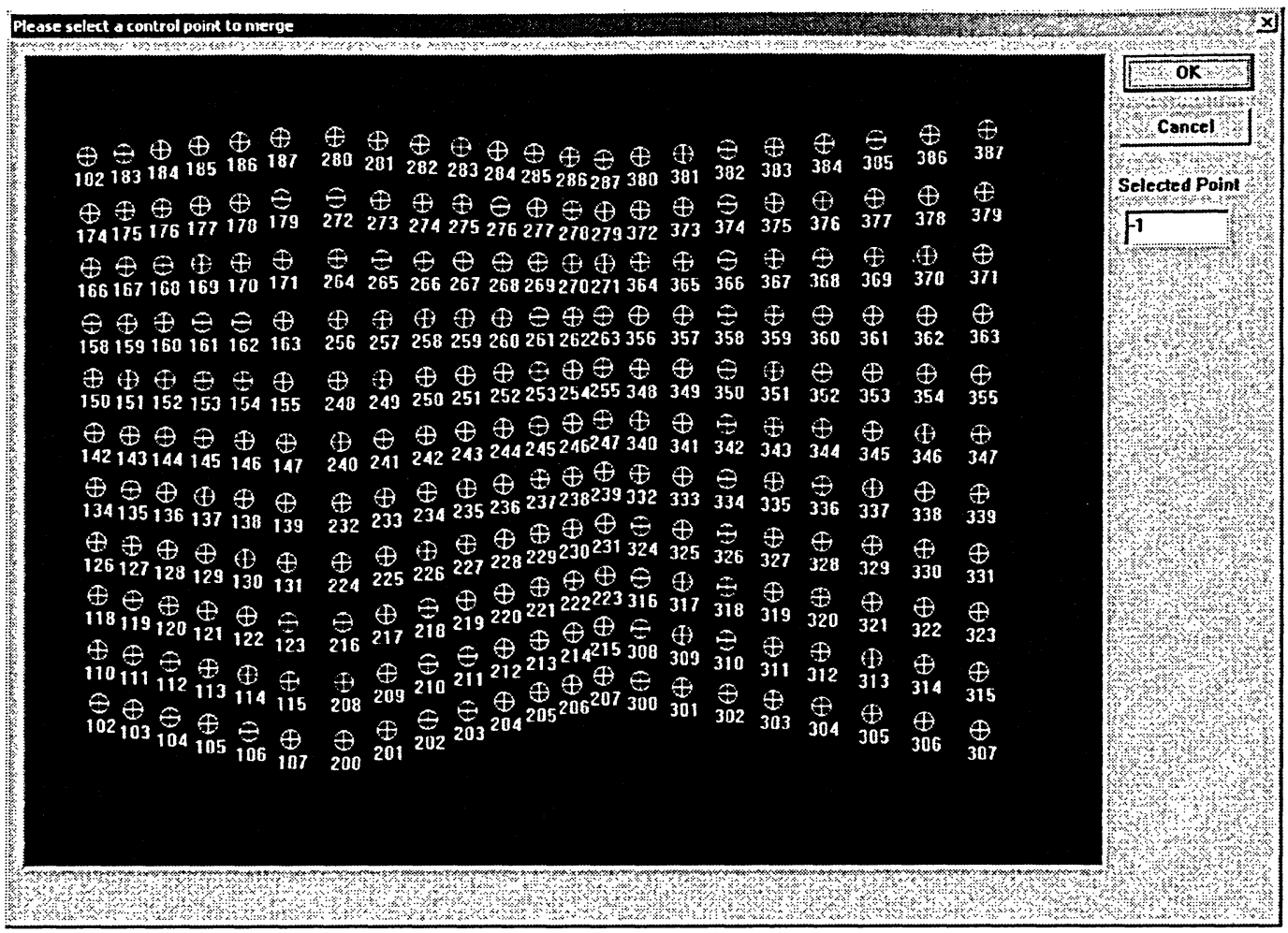

Figure 4-5 GCP selection window used in determining the relationship between

$$
\text { reference points and GCPs }
$$

Another method automatically finds several pairs of such points. Here $\phi$ is defined as orientation angle about $\mathrm{Y}$ axis. Based on the distribution of GCPs and if $\phi$ is not so big, all GCPs can be projected to an image plane as a similar pattern. As shown in Figure 4-5, this pattern consists of 22 columns and 11 rows. In each row, the distance between two neighbour points in $\mathrm{X}$ direction is much greater than it is in $\mathrm{Y}$ direction. On the other hand, in each column, this distance between two neighbour points in $\mathrm{Y}$ direction is much greater than it is in $\mathrm{X}$ direction. These features relationships can be used for automatically finding target points. The following steps describe how to find a reference point next to basic point on the same row in negative $\mathrm{X}$ direction. 
Step1: Define a variable named "mindistance" in order to save the minimum distance between basic point and other reference points. Define a variable named " $\mathrm{nFind}$ " in order to save the found point number.

Step2: Let "mindistance $=10000$ ". This value is greater than the maximum distance between two reference points. So the value of "mindistance" can be substituted in following steps. Let " $n$ Find=-1". This value will be substituted with current point number if it satisfies the condition mentioned in step $<4>$.

Step3: Calculate the differences of $x, y$ between the first reference point and basic point and distance between these two points.

Step4: If values calculated in step $<3>$ satisfy the conditions below:

- the difference of $x$ is smaller than -30 pixels;

- absolute value of $x$ difference $>$ absolute value of $y$ difference;

- the distance between these two points is smaller than "mindistance"

Then

Let "nFind= current point number";

Let "mindistance=the distance between current point and basic point".

Step5: Repeat steps $<3>$ and $<4>$ by changing current point one by one from reference point table until all reference points are involved.

Step6: If "nFind" is not equal to -1 , the value of "nFind" indicates the neighbour point that is on the same row of basic in negative $\mathrm{x}$ direction. 

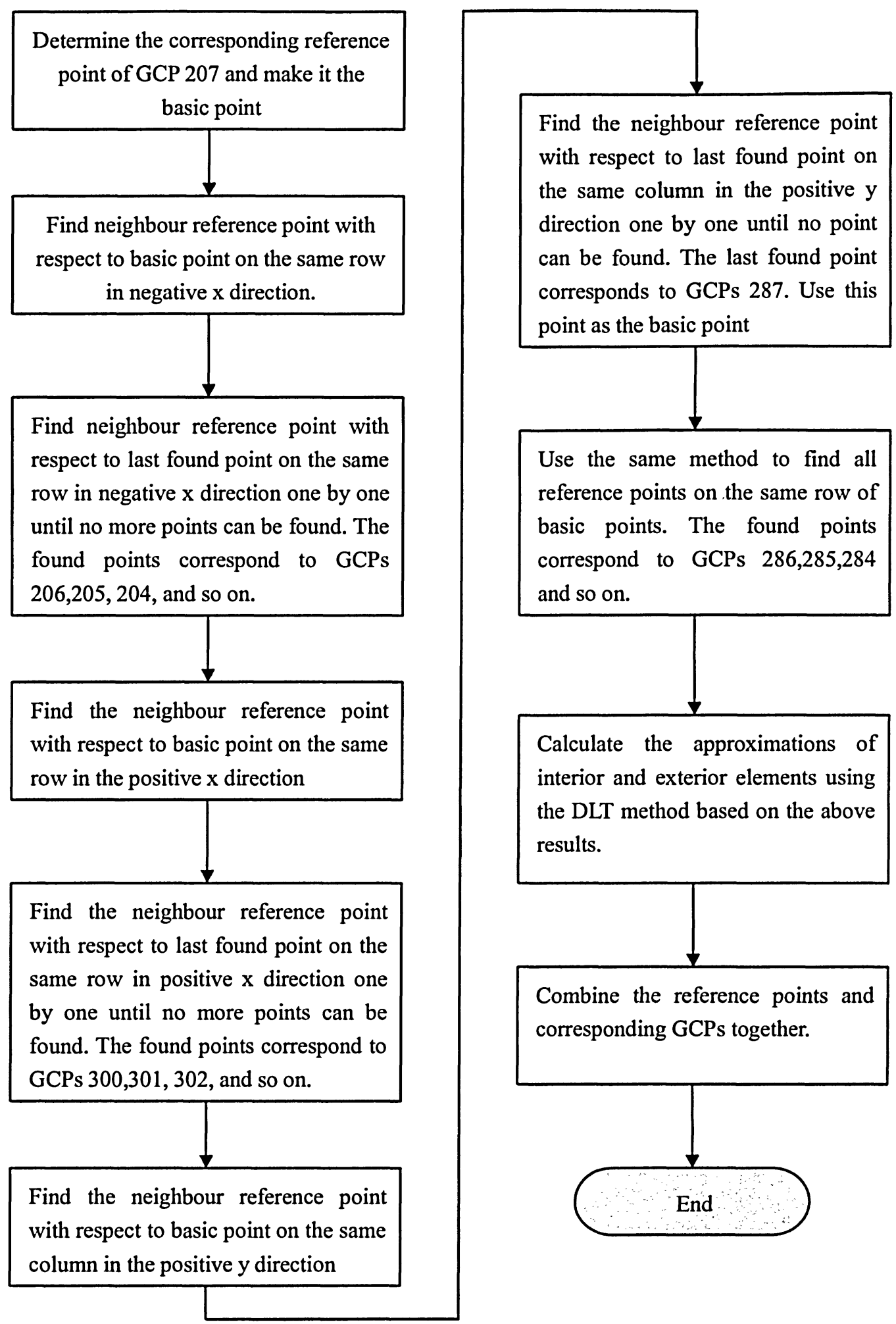

Figure 4-6 Automatically combining reference points and corresponding GCPs. 
Figure 4-6 is the flow chart of automatically combining reference points and corresponding GCPs. The advantage of this method is that it works automatically except specifying the first basic point. The disadvantage of this method is that it cannot work within a big $\phi$ orientation angle. As the operator can easily maintain the camera within a small $\phi$, the second method is advised. In Figure 4-7, the green circles and digital numbers are the result of such an approach.

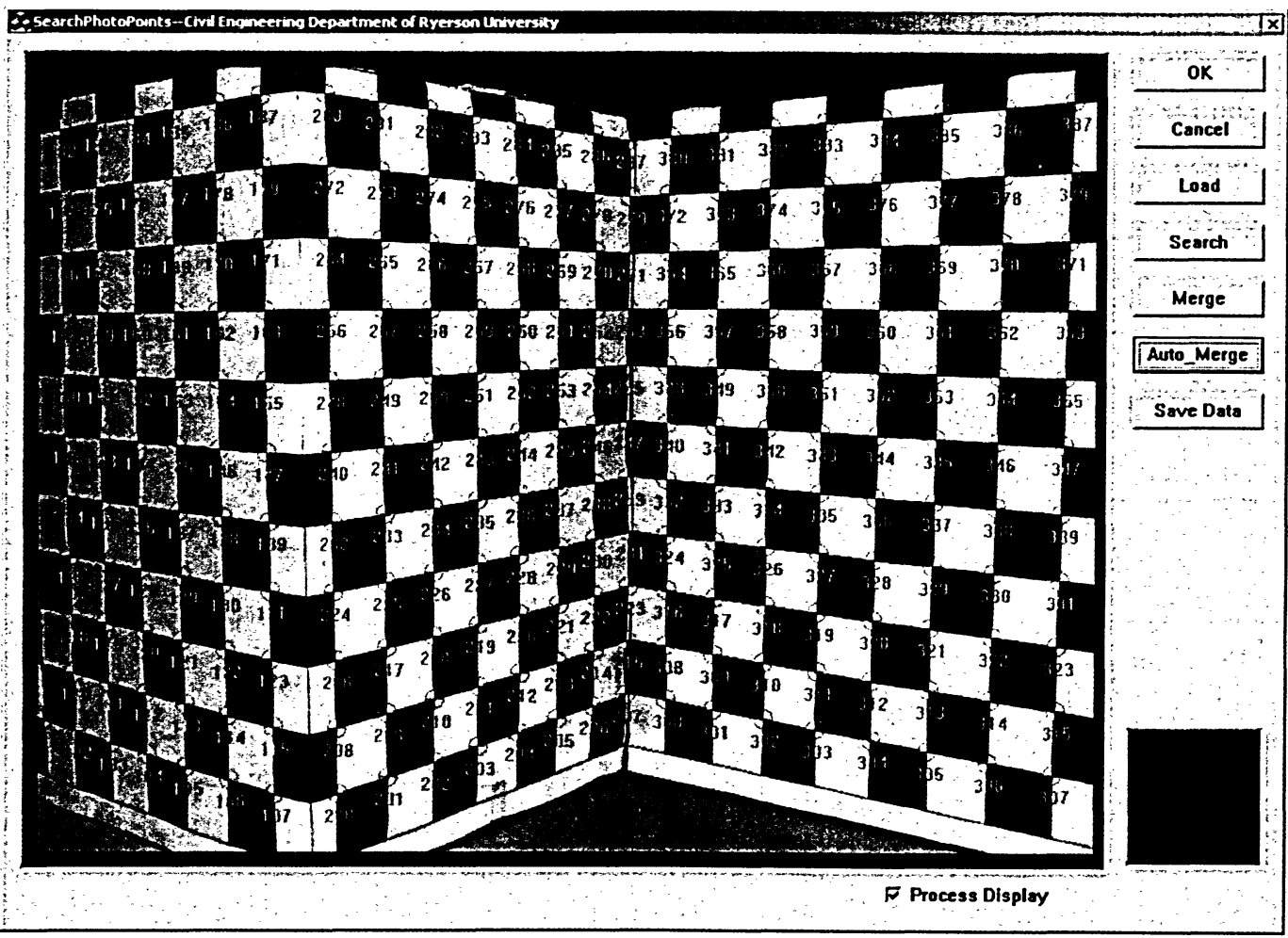

Figure 4-7 An example of combination result

Table 4-1 shows an example of data set computed by this program. This data set is used for camera calibration computing, which will be described in Section 4.2. 
Table 4-1 An example of data set computed by the automatic detection program

\begin{tabular}{|c|c|c|c|c|c|}
\hline $\begin{array}{c}\text { Point } \\
\text { Number }\end{array}$ & $\begin{array}{l}X \text { ground } \\
\text { Coordinates } \\
\text { (m) }\end{array}$ & $\begin{array}{l}\text { Y ground } \\
\text { Coordinates } \\
\text { (m) }\end{array}$ & $\begin{array}{l}\mathrm{Z} \text { ground } \\
\text { Coordinates } \\
\text { (m) }\end{array}$ & $\begin{array}{c}\mathrm{X} \text { image } \\
\text { Coordinates } \\
\text { (pixel) }\end{array}$ & $\begin{array}{c}\text { Y image } \\
\text { Coordinates } \\
\text { (pixel) }\end{array}$ \\
\hline 102 & -0.2366 & -0.1693 & 0.0298 & -1514.9 & -850.0 \\
\hline 103 & -0.2048 & -0.1685 & 0.0476 & -1407.9 & -885.0 \\
\hline 104 & -0.1749 & -0.1687 & 0.0615 & -1294.5 & -922.7 \\
\hline 105 & -0.1442 & -0.1686 & 0.0771 & -1173.6 & -962.0 \\
\hline 106 & -0.1134 & -0.1685 & 0.0926 & -1043.1 & -1003.2 \\
\hline 107 & -0.0827 & -0.1686 & 0.1066 & -901.5 & -1045.8 \\
\hline 110 & -0.2357 & -0.1352 & 0.0299 & -1521.7 & -668.0 \\
\hline$\cdots \cdots$ & $\cdots \cdots$ & $\cdots \cdots$ & $\cdots \cdots$ & $\cdots \cdot \cdots$ & $\cdots \cdots$ \\
\hline 378 & 0.2639 & 0.1361 & -0.0078 & 1345.4 & 886.5 \\
\hline 379 & 0.2954 & 0.1364 & 0.0079 & 1554.6 & 898.8 \\
\hline 380 & 0.0807 & 0.1699 & -0.1056 & 313.0 & 1004.9 \\
\hline 381 & 0.1114 & 0.1698 & -0.0876 & 463.9 & 1019.0 \\
\hline 382 & 0.1415 & 0.1698 & -0.0719 & 621.9 & 1034.0 \\
\hline 383 & 0.1722 & 0.1699 & -0.0567 & 789.8 & 1048.3 \\
\hline 384 & 0.2028 & 0.1699 & -0.0401 & 966.6 & 1064.1 \\
\hline 385 & 0.2334 & 0.1701 & -0.0244 & 1152.9 & 1080.1 \\
\hline 386 & 0.2647 & 0.1706 & -0.0094 & 1350.4 & 1097.9 \\
\hline 387 & 0.2964 & 0.1711 & 0.0076 & 1561.7 & 1116.6 \\
\hline
\end{tabular}

\subsection{Camera Calibration and Generation of a Look Up Table}

This process is used to calibrate digital cameras, compute lens distortion coefficients and analyze errors using the DLT method. The function of adding calibration result into a Look Up Table (LUT) is also included. This is a Microsoft Windows based program coded using Visual $\mathrm{C}++$. Figure 4-8 illustrates the interface of camera calibration program. This program involves the following steps:

Step 1: the loading and displaying positions of reference points; · 
Step2: automatic computation of the calibration result;

Step3: the generation of a LUT.

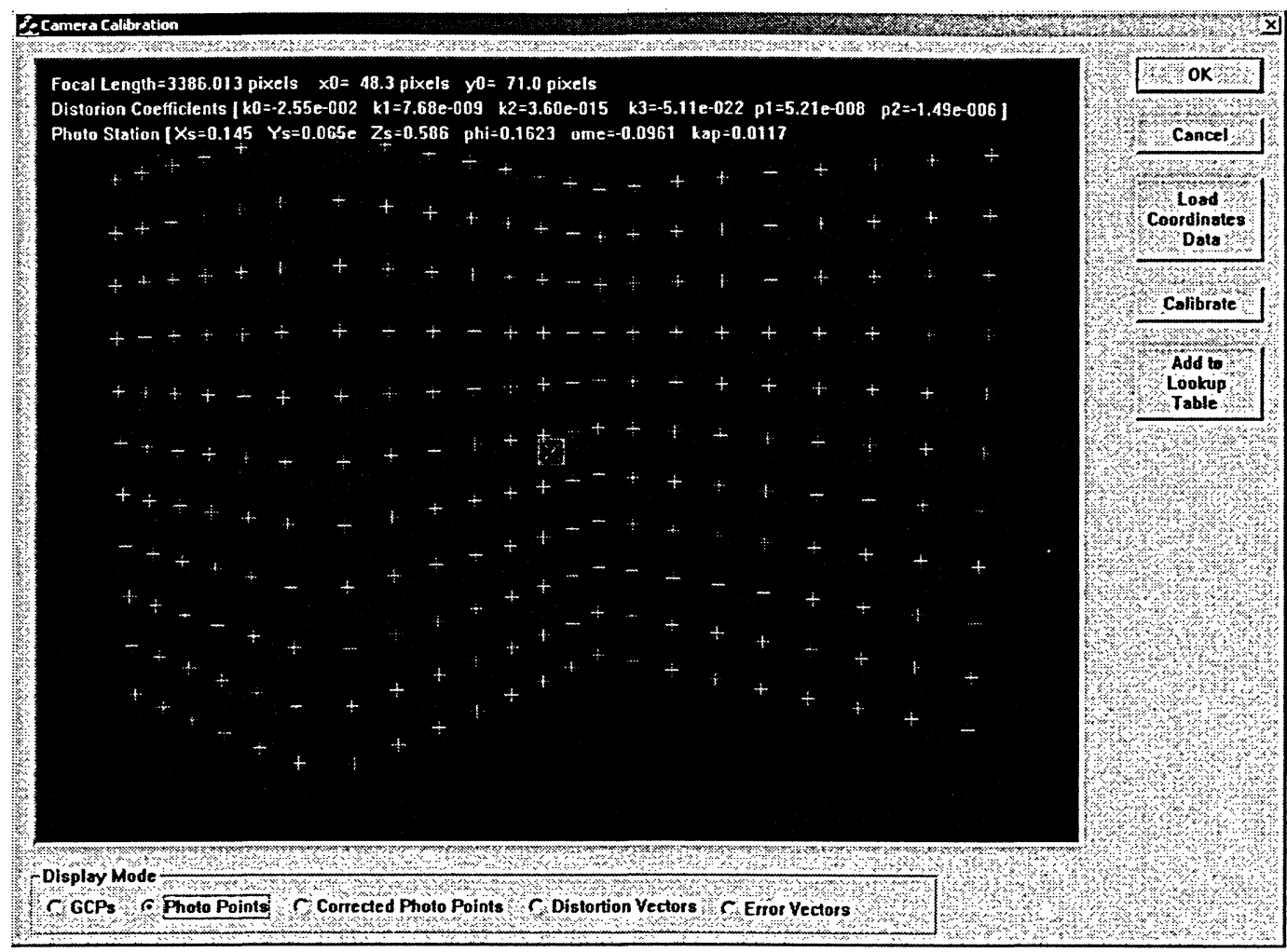

Figure 4-8 The interface of camera calibration program

\subsubsection{Data Processing}

Although the interior elements, exterior elements and distortion coefficients can be computed at the same time, we divide this process into two parts as there is a strong correlation between the focal length and symmetrical distortion coefficient $k_{0}$. This kind of processing method makes the computed result more stable. Figure 4-9 shows the flow chart of data processing. 


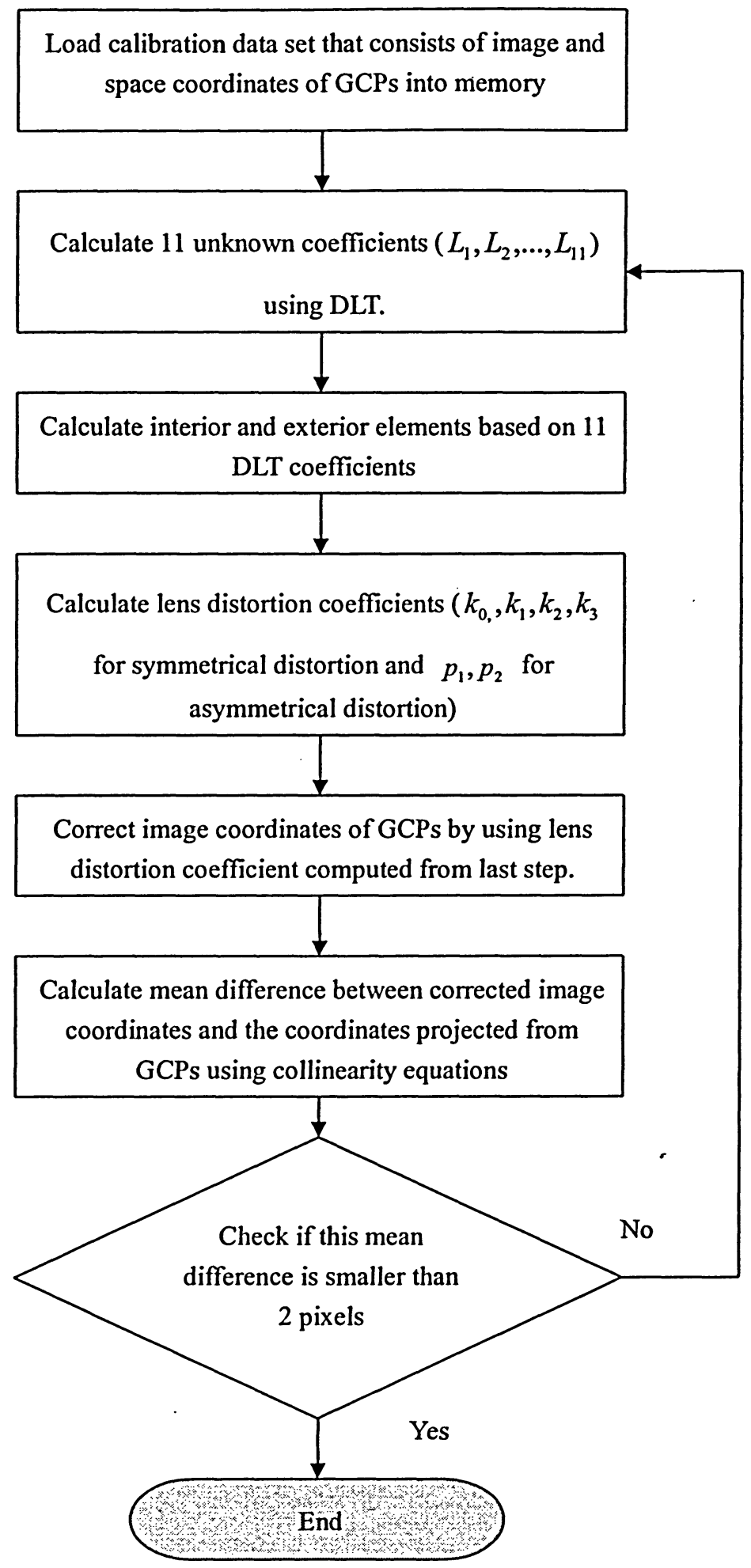

Figure 4-9 The data processing flow chart of camera calibration 


\subsubsection{Result Analysis}

Figure 4-10 shows the result of camera calibration when the actual focal length is

$9.5 \mathrm{~mm}$. The error vectors are also shown in this figure. From this figure, we can see that most errors are smaller than 2 pixels. It means the accuracy of camera calibration is very good. Figure 4-11 shows the distribution of lens distortion. From this figure, we can see that the lens distortion is very big at a small focal length. The correction of lens distortion is, therefore, required.

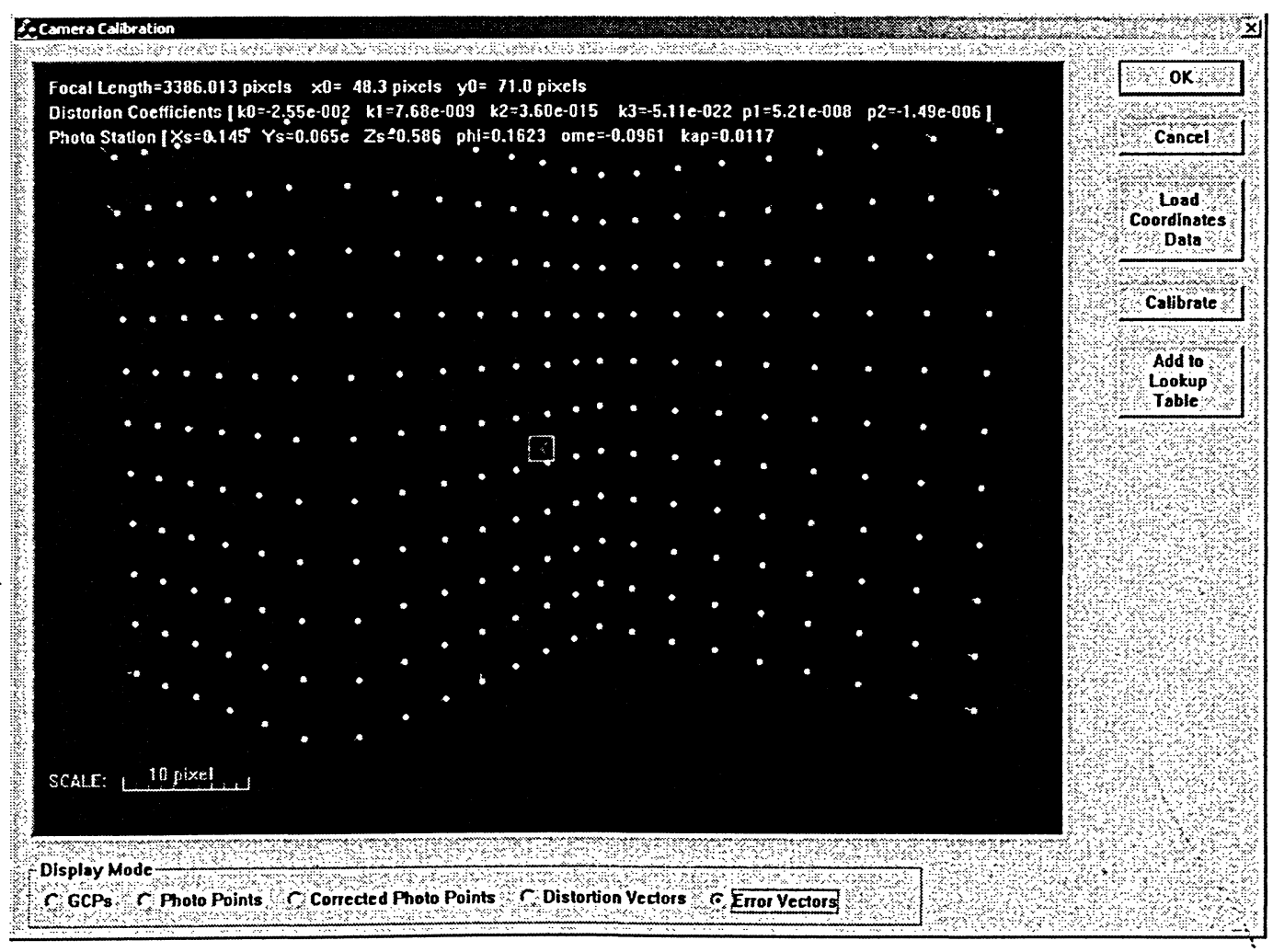

Figure 4-10 The calibration result and error vectors 


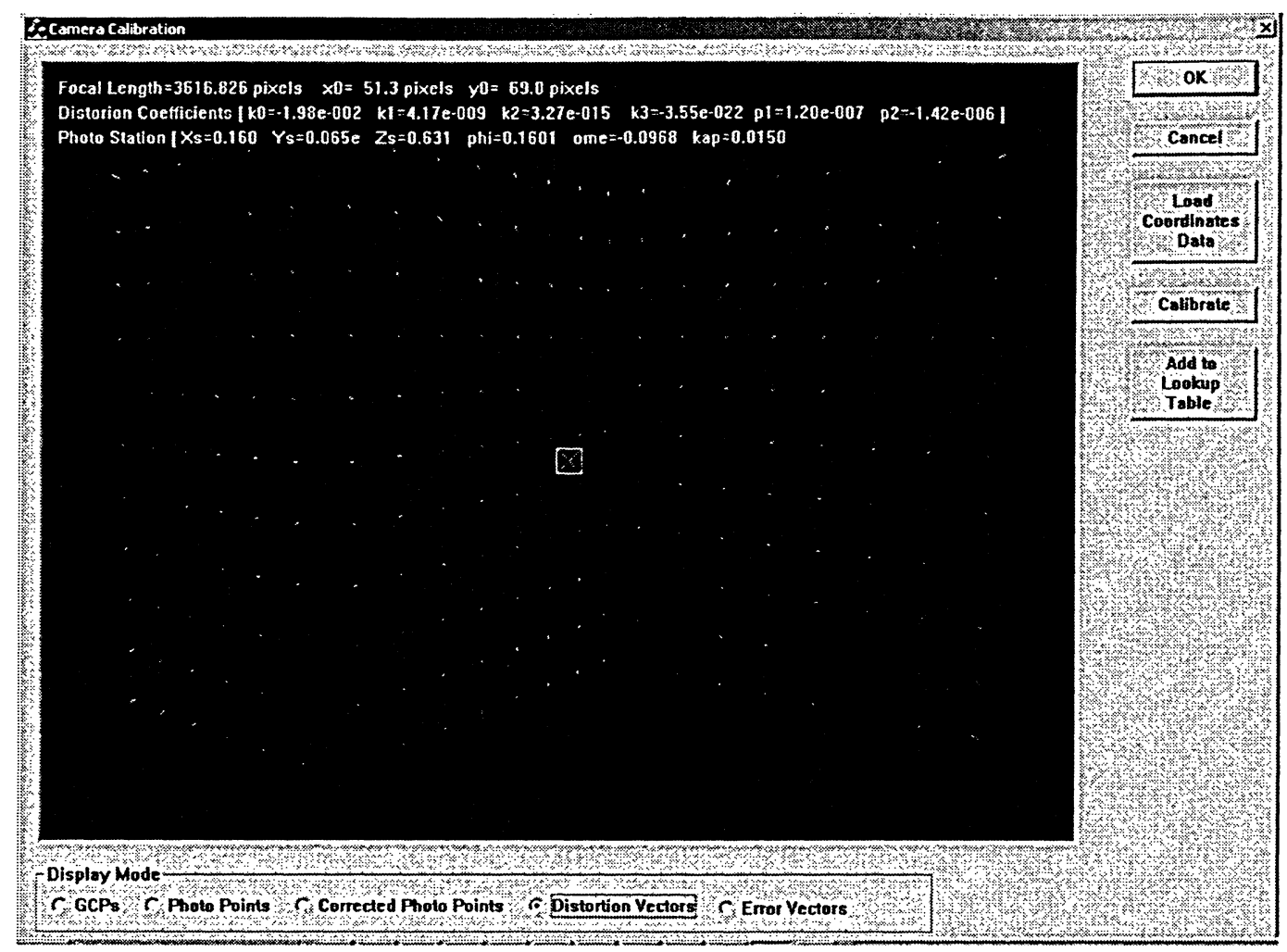

Figure 4-11 The distribution of lens distortion

After calibrating the camera at all actual focal length settings, a LUT can be established. It is indexed by actual focal lengths, which are saved in the first column. These data can be easily used in external orientation program and $3 \mathrm{D}$ reconstruction program. As this lookup table contains 12 columns and is not easy to be shown in this thesis, it is divided into two tables for displaying. Table 4-2 shows the first part of the LUT. There are 6 columns named real focal length, equivalent focal length, $x$ coordinates of principal point, $\mathrm{y}$ coordinate of principal points, mean errors in $\mathrm{x}$ direction and mean errors in y direction in this table. From the mean errors columns, we can see that all these mean errors are less than 2.0 pixels. This means that the accuracy of camera calibration using this method is very good and validates 
photogrammetric processes used in our project.

Table 4-2 The first part of the LUT

\begin{tabular}{|c|c|c|c|c|c|}
\hline $\begin{array}{l}\text { Focal } \\
\text { length } \\
(\mathrm{mm})\end{array}$ & $\begin{array}{c}\text { Equivalent } \\
\text { focal length } \\
\text { (pixels) }\end{array}$ & $\begin{array}{l}\mathrm{x} \text { coordinate of } \\
\text { principal point } \\
\text { (pixels) }\end{array}$ & $\begin{array}{l}\text { y coordinate of } \\
\text { principal point } \\
\text { (pixels) }\end{array}$ & $\begin{array}{c}\text { Mean errors } \\
\text { in } x \text { direction } \\
\text { (pixels) }\end{array}$ & $\begin{array}{c}\text { Mean errors } \\
\text { in y direction } \\
\text { (pixels) }\end{array}$ \\
\hline 8.9 & 3386.0 & 48.3 & 71.0 & 1.2 & 1.0 \\
\hline 10.2 & 3866.8 & 41.1 & 62.9 & 1.3 & 1.2 \\
\hline 11.0 & 4137.4 & 46.1 & 57.4 & 1.3 & 1.1 \\
\hline 11.8 & 4445.2 & 50.1 & 54.9 & 1.0 & 0.7 \\
\hline 12.7 & 4822.2 & 38.2 & 47.9 & 1.3 & 1.1 \\
\hline 13.8 & 5234.9 & 55.0 & 41.3 & 1.0 & 0.5 \\
\hline 15.0 & 5666.8 & 53.3 & 42.4 & 0.6 & 0.5 \\
\hline 16.4 & 6193.6 & 50.2 & 45.0 & 0.6 & 0.5 \\
\hline 18.0 & 6763.3 & 59.3 & 40.2 & 1.0 & 0.7 \\
\hline 19.8 & 7494.5 & 62.2 & 36.8 & 1.2 & 0.8 \\
\hline 21.8 & 8281.0 & 70.6 & 35.6 & 1.3 & 0.9 \\
\hline 24.0 & 9076.7 & 82.8 & 38.8 & 1.3 & 0.9 \\
\hline 26.5 & 9967.9 & 107.4 & 33.2 & 1.3 & 0.9 \\
\hline 29.3 & 11089.7 & 100.2 & 36.1 & 1.5 & 0.9 \\
\hline 32.4 & 12087.9 & 103.4 & 36.6 & 1.2 & 0.8 \\
\hline 35.9 & 13566.5 & 143.1 & 17.7 & 1.4 & 1.0 \\
\hline 40.0 & 14835.5 & 153.0 & 26.5 & 1.4 & 1.0 \\
\hline 44.5 & 16657.9 & 126.5 & 14.4 & 0.9 & 0.7 \\
\hline 49.0 & 18383.4 & 139.7 & 10.9 & 0.9 & 0.7 \\
\hline 53.3 & 19832.3 & 202.6 & 9.3 & 1.5 & 1.0 \\
\hline 57.3 & 21438.1 & 162.7 & 9.7 & 1.5 & 0.9 \\
\hline 61.0 & 23005.4 & 170.9 & 5.1 & 1.0 & 0.8 \\
\hline 64.5 & 24106.7 & 245.4 & -5.7 & 1.5 & 0.9 \\
\hline 68.0 & 25452.1 & 193.5 & -14.9 & 1.1 & 0.8 \\
\hline 71.0 & 26508.8 & 198.4 & -19.8 & 1.0 & 0.8 \\
\hline 74.0 & 27332.8 & 264.0 & -15.4 & 1.5 & 0.9 \\
\hline 77.0 & 29882.1 & 290.9 & -2.3 & 1.4 & 1.1 \\
\hline 80.0 & 32496.6 & 263.0 & -19.8 & 2.0 & 1.2 \\
\hline 83.0 & 33619.4 & 269.2 & -40.6 & 1.5 & 1.1 \\
\hline 86.0 & 34343.5 & 263.7 & -11.1 & 1.6 & 1.0 \\
\hline 89.0 & 35056.5 & 257.6 & -20.4 & 1.6 & 1.0 \\
\hline
\end{tabular}

Table 4-3 shows the second part of the LUT. There are 7 columns named focal length, $\mathrm{K} 0, \mathrm{~K} 1, \mathrm{~K} 2, \mathrm{~K} 3, \mathrm{P} 1$ and $\mathrm{P} 2 . \mathrm{K} 0, \mathrm{~K} 1, \mathrm{~K} 2$ and $\mathrm{K} 3$ are symmetrical distortion 
coefficients where P1 and P2 are asymmetrical distortion coefficients correspond to each focal length setting. All these coefficients are used for correcting image coordinates.

Table 4-3 The second part of the LUT

\begin{tabular}{|c|c|c|c|c|c|c|}
\hline $\begin{array}{l}\text { Focal } \\
\text { length } \\
(\mathrm{mm})\end{array}$ & K0 & K1 & $\mathrm{K} 2$ & $\mathrm{~K} 3$ & P1 & P2 \\
\hline 8.90 & $-2.548277 \mathrm{e}-2$ & $7.680151 e-9$ & $3.604053 e-015$ & $-5.113495 \mathrm{e}-22$ & $5.213991 \mathrm{e}-8$ & $-1.487180 e-6$ \\
\hline 10.20 & $-1.343463 e-2$ & $1.207450 \mathrm{e}-9$ & $2.959306 \mathrm{e}-15$ & $-2.769483 \mathrm{e}-22$ & $4.546367 \mathrm{e}-9$ & $-1.107249 \mathrm{e}-6$ \\
\hline 11.00 & $-7.629539 \mathrm{e}-3$ & $-2.406454 \mathrm{e}-9$ & $3.753245 \mathrm{e}-15$ & $-4.184476 \mathrm{e}-22$ & $1.714209 \mathrm{e}-7$ & $-9.580816 e-7$ \\
\hline 11.80 & $-4.216220 \mathrm{e}-3$ & $-3.107829 \mathrm{e}-9$ & $2.864318 \mathrm{e}-15$ & $-2.474525 \mathrm{e}-22$ & $1.285686 \mathrm{e}-7$ & $-5.993249 \mathrm{e}-7$ \\
\hline 12.70 & $-5.003205 e-4$ & $-4.404231 e-9$ & $2.287725 \mathrm{e}-15$ & $-1.102565 \mathrm{e}-22$ & $6.772650 \mathrm{e}-9$ & $-4.596684 \mathrm{e}-7$ \\
\hline 13.80 & $2.273171 \mathrm{e}-3$ & $-5.185475 e-9$ & $1.979763 e-15$ & $-8.214143 e-23$ & $1.759269 \mathrm{e}-7$ & $-1.594175 \mathrm{e}-7$ \\
\hline 15.00 & $2.680209 \mathrm{e}-3$ & $-4.238929 e-9$ & $1.229739 \mathrm{e}-15$ & $4.939702 \mathrm{e}-24$ & $2.716129 \mathrm{e}-8$ & $-8.853269 e-8$ \\
\hline 16.40 & $3.298657 \mathrm{e}-3$ & $-4.559726 e-9$ & $1.136812 \mathrm{e}-15$ & $4.632399 \mathrm{e}-23$ & $-6.110697 \mathrm{e}-8$ & $-1.073971 e-7$ \\
\hline 18.00 & $3.917346 \mathrm{e}-3$ & $-4.436498 \mathrm{e}-9$ & $9.719586 e-16$ & $2.854615 \mathrm{e}-23$ & $-1.921688 \mathrm{e}-7$ & $3.383763 e-8$ \\
\hline 19.80 & $4.143272 e-3$ & $-4.193232 e-9$ & $6.893298 \mathrm{e}-16$ & $7.678896 \mathrm{e}-23$ & $-2.439397 \mathrm{e}-7$ & $3.858705 e-8$ \\
\hline 21.80 & $4.490033 e-3$ & $-4.283552 e-9$ & $6.579841 \mathrm{e}-16$ & $7.333059 \mathrm{e}-23$ & $-2.497901 \mathrm{e}-7$ & $4.854264 \mathrm{e}-8$ \\
\hline 24.00 & $4.521592 \mathrm{e}-3$ & $-4.475889 \mathrm{e}-9$ & $7.224015 e-16$ & $7.882564 \mathrm{e}-23$ & $-2.630362 \mathrm{e}-7$ & $4.309004 e-8$ \\
\hline 26.50 & $6.401494 \mathrm{e}-3$ & $-5.884621 \mathrm{e}-9$ & $9.611868 \mathrm{e}-16$ & $5.863000 \mathrm{e}-23$ & $3.257969 \mathrm{e}-8$ & $1.270459 \mathrm{e}-7$ \\
\hline 29.30 & $4.550779 \mathrm{e}-3$ & $-4.140812 \mathrm{e}-9$ & $5.156072 \mathrm{e}-16$ & $9.814533 \mathrm{e}-23$ & $-2.701871 \mathrm{e}-7$ & $5.169833 \mathrm{e}-8$ \\
\hline 32.40 & $6.273380 \mathrm{e}-3$ & $-6.304394 e-9$ & $1.527833 \mathrm{e}-15$ & $-8.328108 \mathrm{e}-23$ & $-6.066844 \mathrm{e}-8$ & $1.349531 \mathrm{e}-7$ \\
\hline 35.90 & $6.623646 \mathrm{e}-3$ & $-5.545837 \mathrm{e}-9$ & $7.712541 \mathrm{e}-16$ & $5.535024 \mathrm{e}-23$ & $3.209292 \mathrm{e}-8$ & $1.176042 \mathrm{e}-7$ \\
\hline 40.00 & $6.610996 \mathrm{e}-3$ & $-5.624672 \mathrm{e}-9$ & $7.510113 \mathrm{e}-16$ & $7.955908 \mathrm{e}-23$ & $4.481964 \mathrm{e}-8$ & $1.482310 \mathrm{e}-7$ \\
\hline 44.50 & $6.783784 \mathrm{e}-3$ & $-4.731228 \mathrm{e}-9$ & $-3.574572 e-17$ & $2.205129 \mathrm{e}-22$ & $8.133374 \mathrm{e}-8$ & $1.780505 e-7$ \\
\hline 49.00 & $6.969207 \mathrm{e}-3$ & $-4.828310 \mathrm{e}-9$ & $1.424250 \mathrm{e}-17$ & $2.043689 \mathrm{e}-22$ & $8.450856 \mathrm{e}-8$ & $1.634358 \mathrm{e}-7$ \\
\hline 53.30 & $6.165193 e-3$ & $-4.651579 \mathrm{e}-9$ & $2.063586 \mathrm{e}-16$ & $1.622306 \mathrm{e}-22$ & $6.517438 \mathrm{e}-8$ & $1.431608 \mathrm{e}-7$ \\
\hline 57.30 & $4.649887 \mathrm{e}-3$ & $-4.325709 \mathrm{e}-9$ & $7.197468 \mathrm{e}-16$ & $4.265881 \mathrm{e}-23$ & $-1.700701 \mathrm{e}-7$ & $2.870408 \mathrm{e}-8$ \\
\hline 61.00 & $6.719039 \mathrm{e}-3$ & $-4.621320 e-9$ & $-8.421762 e-17$ & $2.253197 \mathrm{e}-22$ & $9.855400 \mathrm{e}-8$ & $9.566256 \mathrm{e}-8$ \\
\hline 64.50 & $6.017923 e-3$ & $-4.597585 e-9$ & $2.779844 \mathrm{e}-16$ & $1.371157 \mathrm{e}-22$ & $7.461723 e-8$ & $9.292105 \mathrm{e}-008$ \\
\hline 68.00 & $6.575142 \mathrm{e}-3$ & $-5.013193 e-9$ & $3.790398 \mathrm{e}-16$ & $1.276963 \mathrm{e}-22$ & $1.130200 \mathrm{e}-7$ & $1.323985 \mathrm{e}-007$ \\
\hline 71.00 & $6.583627 e-3$ & $-4.965151 \mathrm{e}-9$ & $2.549411 \mathrm{e}-16$ & $1.646437 \mathrm{e}-22$ & $1.101347 \mathrm{e}-7$ & $1.323462 \mathrm{e}-007$ \\
\hline 74.00 & $5.747952 \mathrm{e}-3$ & $-4.269108 \mathrm{e}-9$ & $1.039040 \mathrm{e}-16$ & $1.723795 \mathrm{e}-22$ & $8.726944 \mathrm{e}-8$ & $1.125900 \mathrm{e}-007$ \\
\hline 77.00 & $6.263798 \mathrm{e}-3$ & $-5.931665 \mathrm{e}-9$ & $1.095286 \mathrm{e}-15$ & $2.898884 \mathrm{e}-23$ & $1.108723 e-8$ & $1.549589 \mathrm{e}-007$ \\
\hline 80.00 & $6.411615 \mathrm{e}-3$ & $-5.560378 \mathrm{e}-9$ & $7.245110 \mathrm{e}-16$ & $1.302762 \mathrm{e}-22$ & $-9.173419 \mathrm{e}-8$ & $4.005601 \mathrm{e}-007$ \\
\hline 83.00 & $7.253849 \mathrm{e}-3$ & $-6.881624 \mathrm{e}-9$ & $1.279339 \mathrm{e}-15$ & $5.931455 \mathrm{e}-23$ & $-2.796813 \mathrm{e}-8$ & $4.532883 \mathrm{e}-007$ \\
\hline 86.00 & $6.239232 \mathrm{e}-3$ & $-5.576970 \mathrm{e}-9$ & $7.073641 \mathrm{e}-16$ & $1.428051 \mathrm{e}-22$ & $-4.307938 \mathrm{e}-8$ & $2.939848 \mathrm{e}-007$ \\
\hline 89.00 & $6.734328 \mathrm{e}-3$ & $-6.279720 e-9$ & $1.213661 \mathrm{e}-15$ & $7.264410 \mathrm{e}-24$ & $-5.147569 e-8$ & $4.237722 \mathrm{e}-007$ \\
\hline
\end{tabular}




\subsection{Chapter Summary}

This chapter presents a digital camera calibration solution in this study. Two software programs are introduced in this chapter. The first program is for automatically detecting image coordinates corresponding to GCPs that can be used for camera calibration. Image matching and LSM are employed in this program. Image matching is used for obtaining initial positions of corresponded image points (reference points) where LSM is used for computing accurate positions by correcting geometric distortion, illumination and reflectance differences. This program also provides two functions to combine GCPs and corresponded reference points. The result of this program can be loaded as an input file used for camera calibration.

The second program is a camera calibration program. The distribution of errors and distortions are shown in this program. It also provides a function to add the calibration result into a LUT, which can be used in other photogrammetric program. The corresponding results indicate that it is a rigid camera calibration program because the interior and exterior elements computed by this program are stable and mean errors of image coordinates are less than 2 pixels.

Both of these two programs are easy to use since good graphical interfaces are provided and most operations can be completed automatically. The objective of developing these programs is realized. 


\section{CASE STUDY OF EXTERNAL ORIENTATION AND GCPS COLLECTION}

This chapter describes the designed study of external orientation and GCPs collection. Three software programs are developed for this study. The objective of the first and second program is to complete GCPs collection using methodologies mentioned in Section 3.2.3. These collected GCPs will be inputted to the second program. The third is an external orientation program used for calculating exterior elements of digital images, which are used for $3 D$ reconstruction.

\subsection{GIS Data Transformation Program}

As mentioned in Section 3.2, external orientation cannot be processed without GCPs. This study presents a new method to collect GCPs from an existing GIS data set. Firstly, the existing GIS data, which is written in AutoCAD 2005 drawing format (.dwg), should be transformed to a special format defined in Section 3.2.3 that can be used in developed GCPs collection program. This transformation includes two steps. The first step is transforming the old GIS data from drawing format to corresponding DXF format by using AutoCAD program. The second step is extracting and transforming needed data from DXF format to our own graphic format (.gph). The designed purpose of GIS data transformation program is to complete the second step. Figure 5-1 shows the flow chart of GIS data transformation program. 


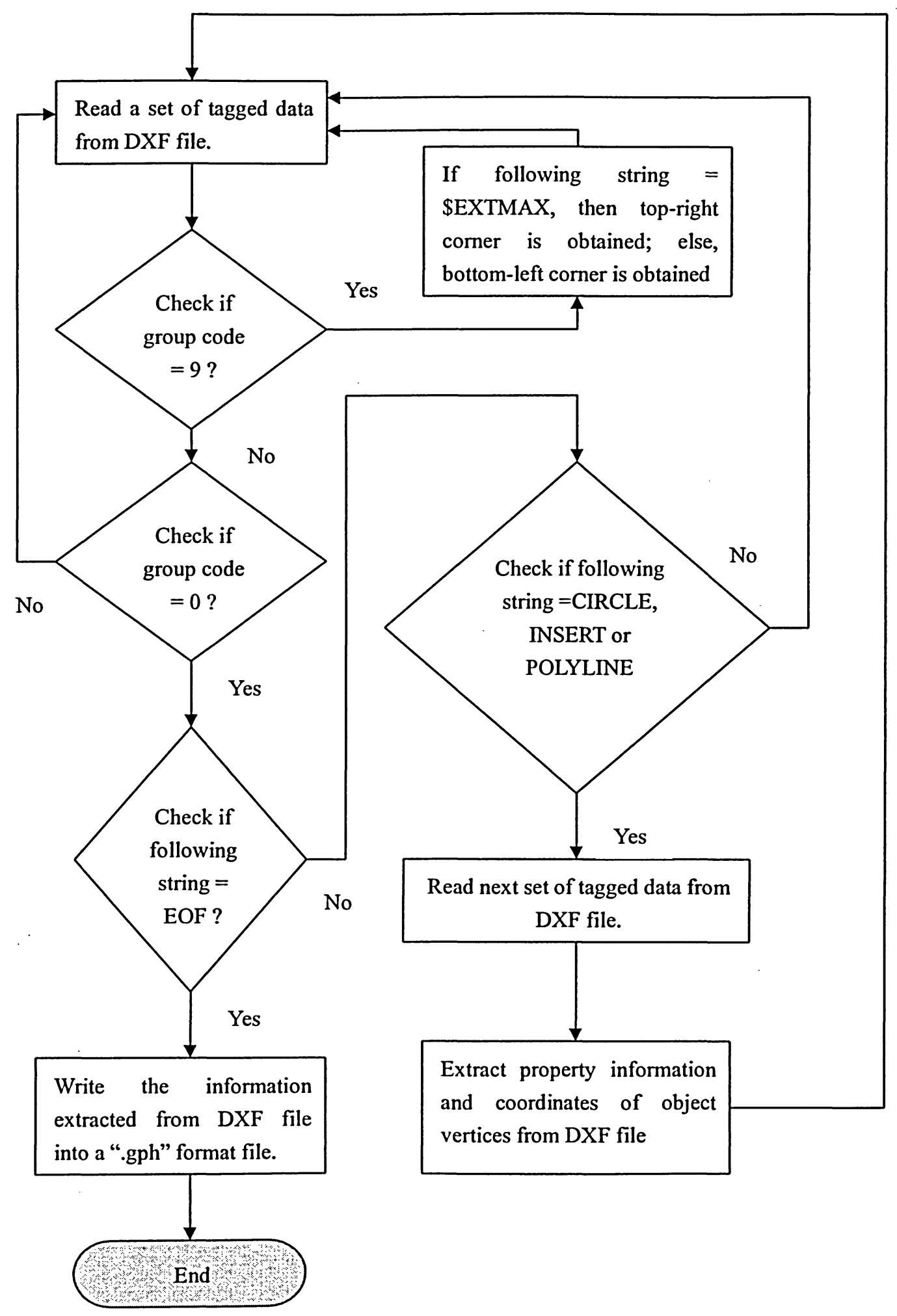

Figure 5-1 Flow chart of GIS data transformation 


\subsection{GCPs Collection Program}

This program is used for collecting GCPs from the field based on GIS and GPS technology. It is a Microsoft Windows program coded by Visual C++. Figure 5-2 shows the interface of this program.

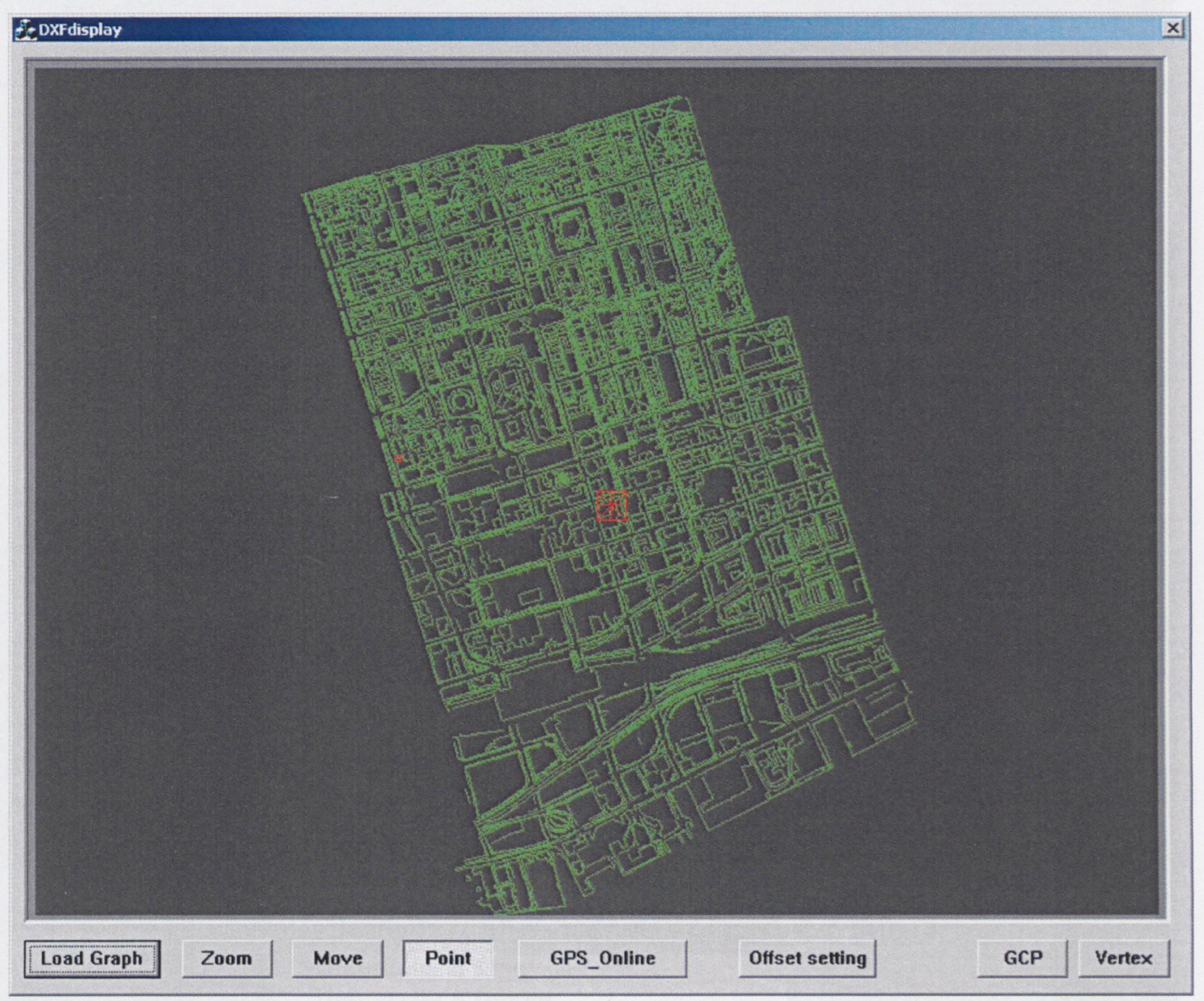

Figure 5-2 The interface of GCPs collection program

This program provides three main functions that help operators collect GCPs from graphics data transformed from existing GIS data. The first function is graphics displaying. After loading graphics document, it is easy to display, zoom and move these graphics data on screen window. The positions of vertices and collected GCPs 
can also be displayed as rectangles with different colours. Figure 5-3 shows an example of zoomed graphics. Vertices are also shown as blue rectangles on the screen.

This function helps operators easily determine which vertex can be chosen as a GCP.

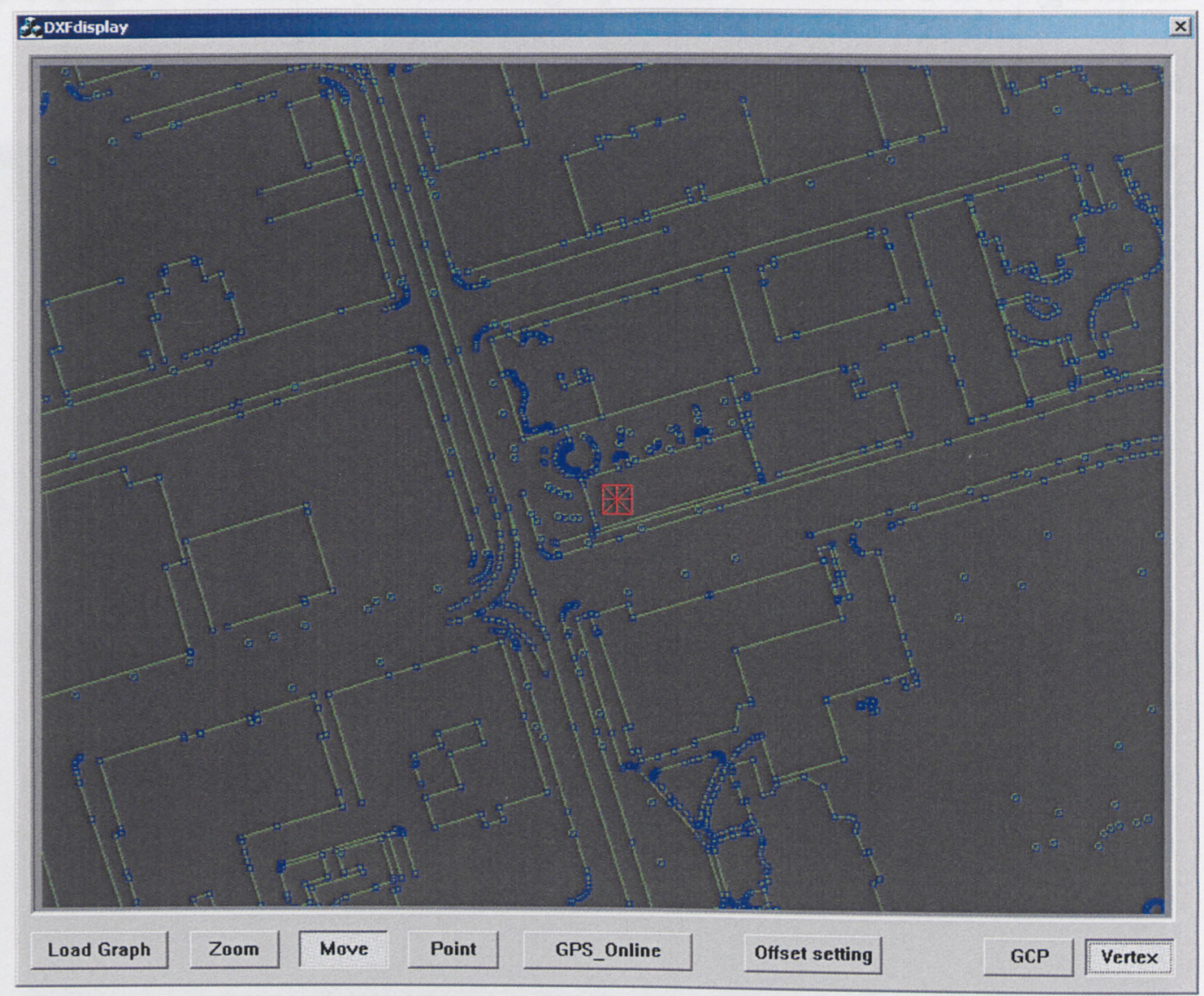

Figure 5-3 Zoomed graphics with vertices displayed

The second main function is GPS navigation. After initializing the status of serial port and set GPS receiver on National Marine Electronics Association (NMEA) mode, this function receives GPS signals every 100 milliseconds. The coordinates of GPS receiver are transformed from a geodetic system to a Modified Transverse Mercator (MTM) system in order to correspond to the local GIS coordinates system. The centre point of displaying area is set to this GPS position so that the graphics data of working 
area can be always displayed on the screen with an appropriate scale. A defect of this function is that GPS signals may be interrupted by some high structures so that the position of GPS receiver cannot be obtained everywhere. An aiding function is used for solving this problem. As shown in Figure 5-4, the coordinates of some intersections are stored in a document and can be selected from a list box of a pop up window. The coordinates of selected intersection will be loaded and set as the centre point. This aiding function is useful when there is no GPS signal.

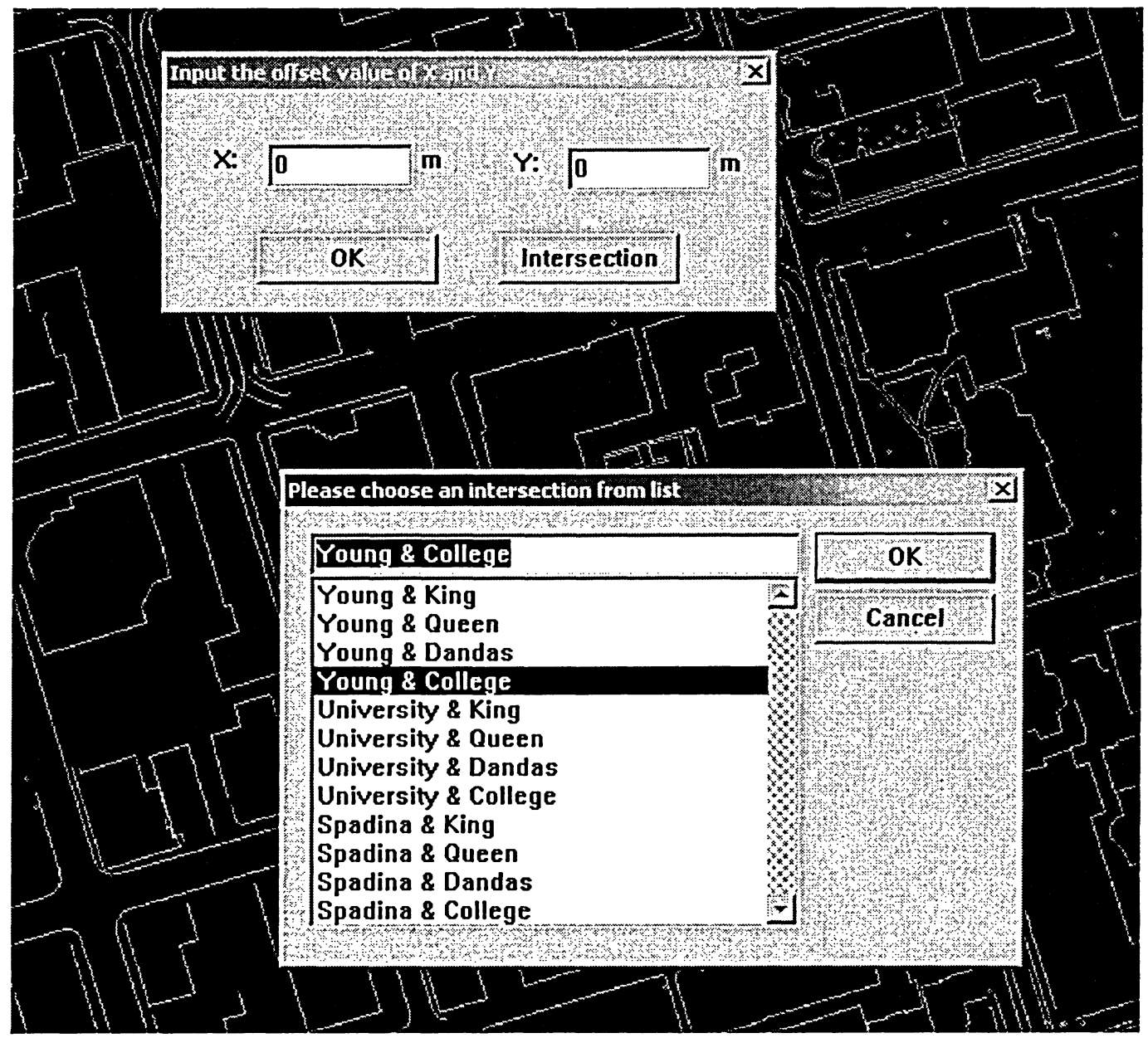

Figure 5-4 Select an intersection as centre point of displayed graphics

The third main function is collecting GCPs from GIS data. GPS receiver gives the 
centre point of working field. The following steps describe the process of how to collect a GCP.

Step1: The displaying, zooming and roaming of graphics map and all vertices.

Step2: The selection of interest vertex.

Step3: The point ID editing of interest vertex. As shown in Figure 5-5, a pop up window shows 3D coordinates of current vertex and default point ID. The point ID can be changed and saved as desired.

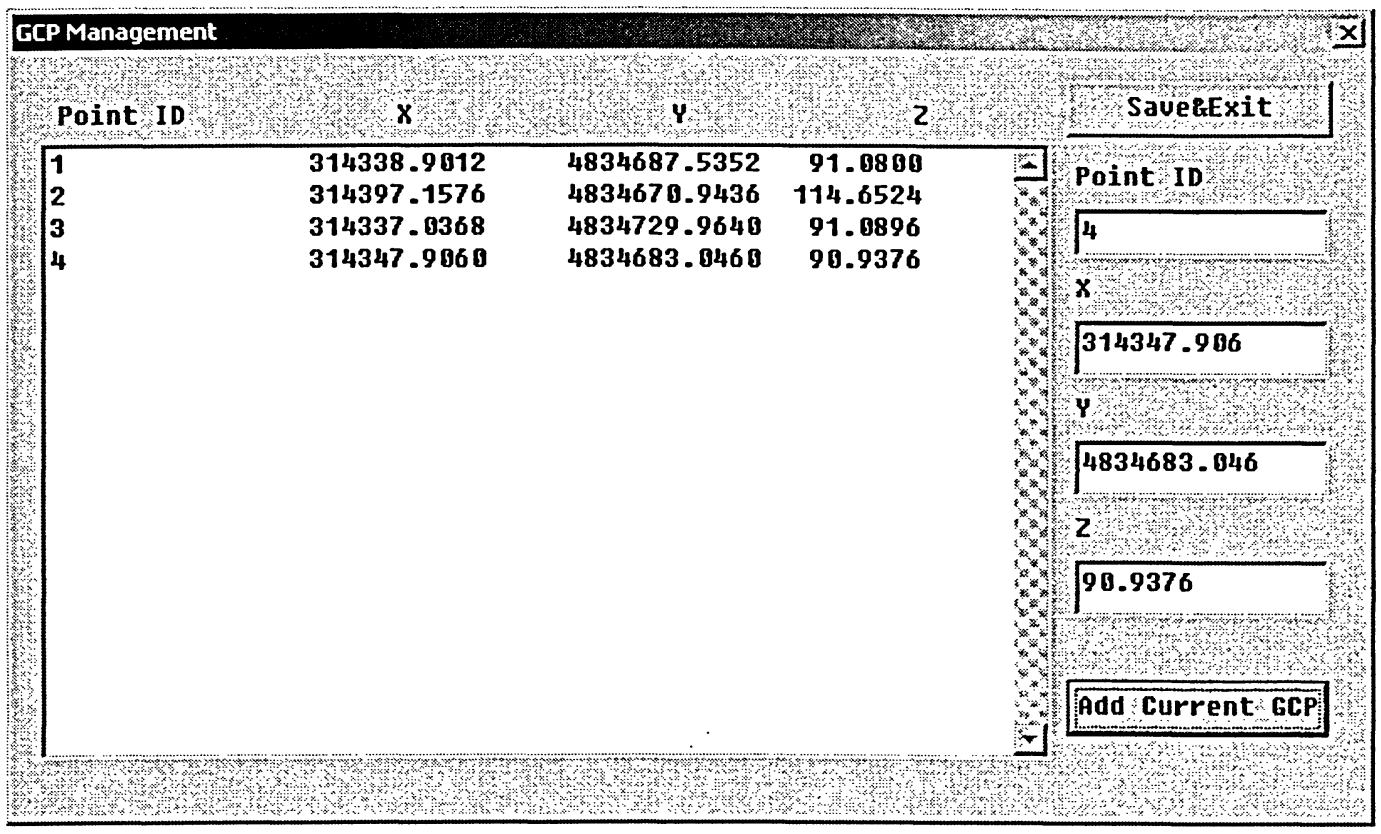

Figure 5-5 GCPs management window

This program provides a good graphical interface that makes it easy to collect GCPs from GIS data that are located in the working area. For a skilled operator, this processing needs no more than 20 minutes. In contrast to traditional methods, such as aero-triangulation or using high accuracy GPS receiver, it has a significant advantage. The accuracy of such vertices is smaller than $0.1 \mathrm{~m}$, which satisfies the demand of 
underground utilities mapping.

\subsection{External Orientation Program}

The purpose of this program is the calculation of the exterior elements of digital images. It is a Microsoft Windows program coded by Visual $\mathrm{C}++$. Figure 5-6 illustrates the interface of this program. There are three sub windows in this program. The biggest window is for displaying the digital image. The measurement of image coordinates works in this window. The top-right window is for displaying full digital image with a small scale. A green rectangle indicates corresponding area displayed in the biggest window. The bottom-right window lists the GCPs imported from GCPs document created from GCPs collection program. The following information indicates the status of GCPs. "Available" means this GCP is involved in calculating the exterior elements. "Unavailable" means it is not used. This status can be changed by double-clicking left mouse button after moving mouse cursor on the corresponding item. As mentioned in Section 3.2.2, collinearity equations are used for external orientation. Since space coordinates of GCPs used in this program are already obtained from GIS data, the main function of this program is providing a user interface where image coordinates of GCPs can be measured and for calculating the exterior elements. 


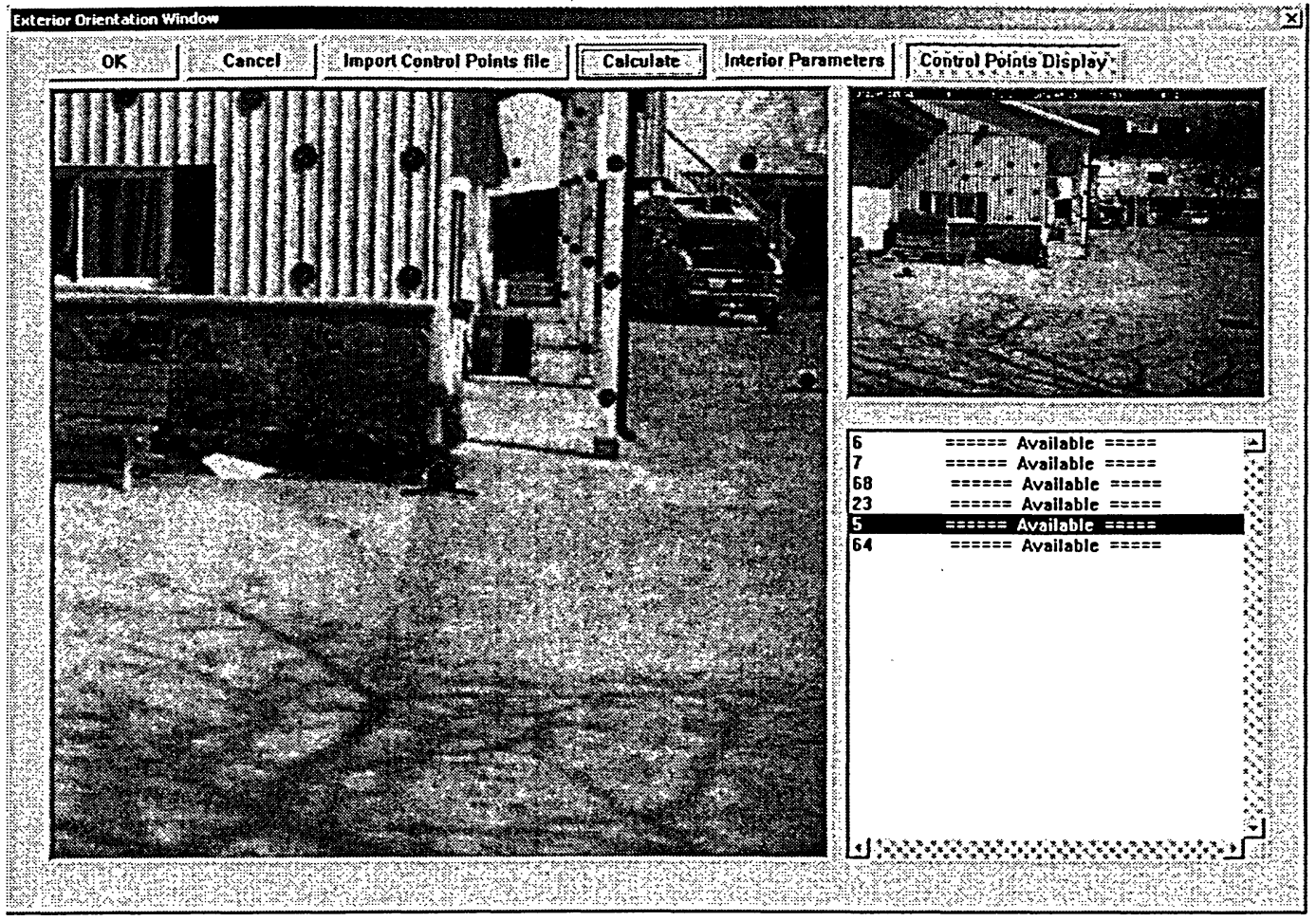

Figure 5-6 The interface of external orientation

The calculation of external orientation cannot proceed without image coordinates corresponding to GCPs. The measurement of image coordinates is divided into following steps:

Step1: The selection of a target GCP.

Step2: The determination of the rough image position corresponding to the target GCP that can be used for displaying the sub image in the measurement window.

Step3: The measurement of image coordinates corresponding to the target GCP.

After both GCPs and their corresponding image coordinates are obtained, the calculating of external orientation can be processed. Figure 5-7 shows the flow chart 
of this processing.

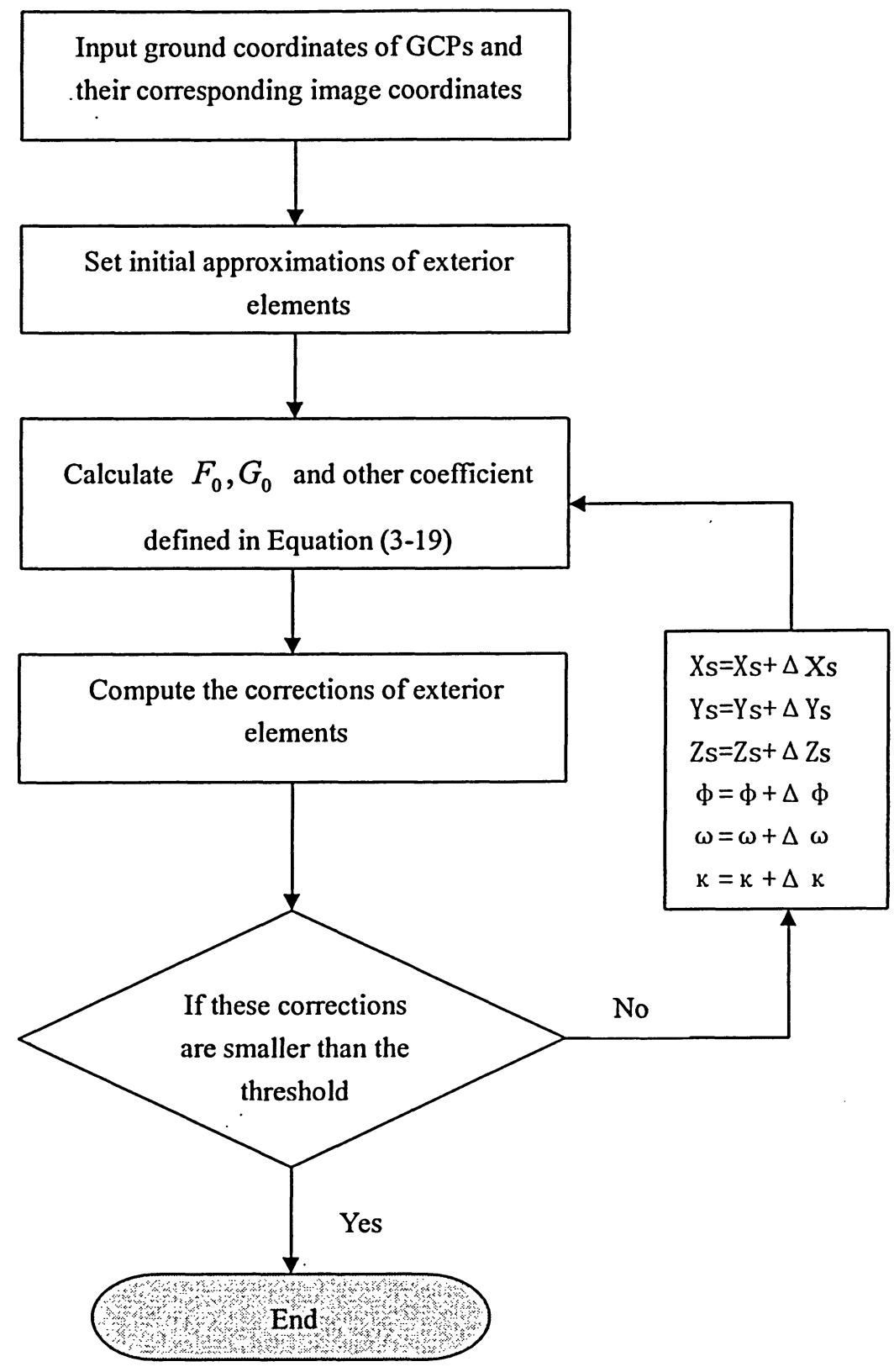

Figure 5-7 Flow chart of calculating exterior elements

The calculating results and errors of GCPs will be automatically saved and displayed in a pop-up window as shown in Figure 5-8. If large errors are found in the error list, 
it means that wrong image coordinates may have been measured. Such image coordinates must be measured again and such mistakes can be corrected. Erroneous ground control coordinates could cause similar errors.

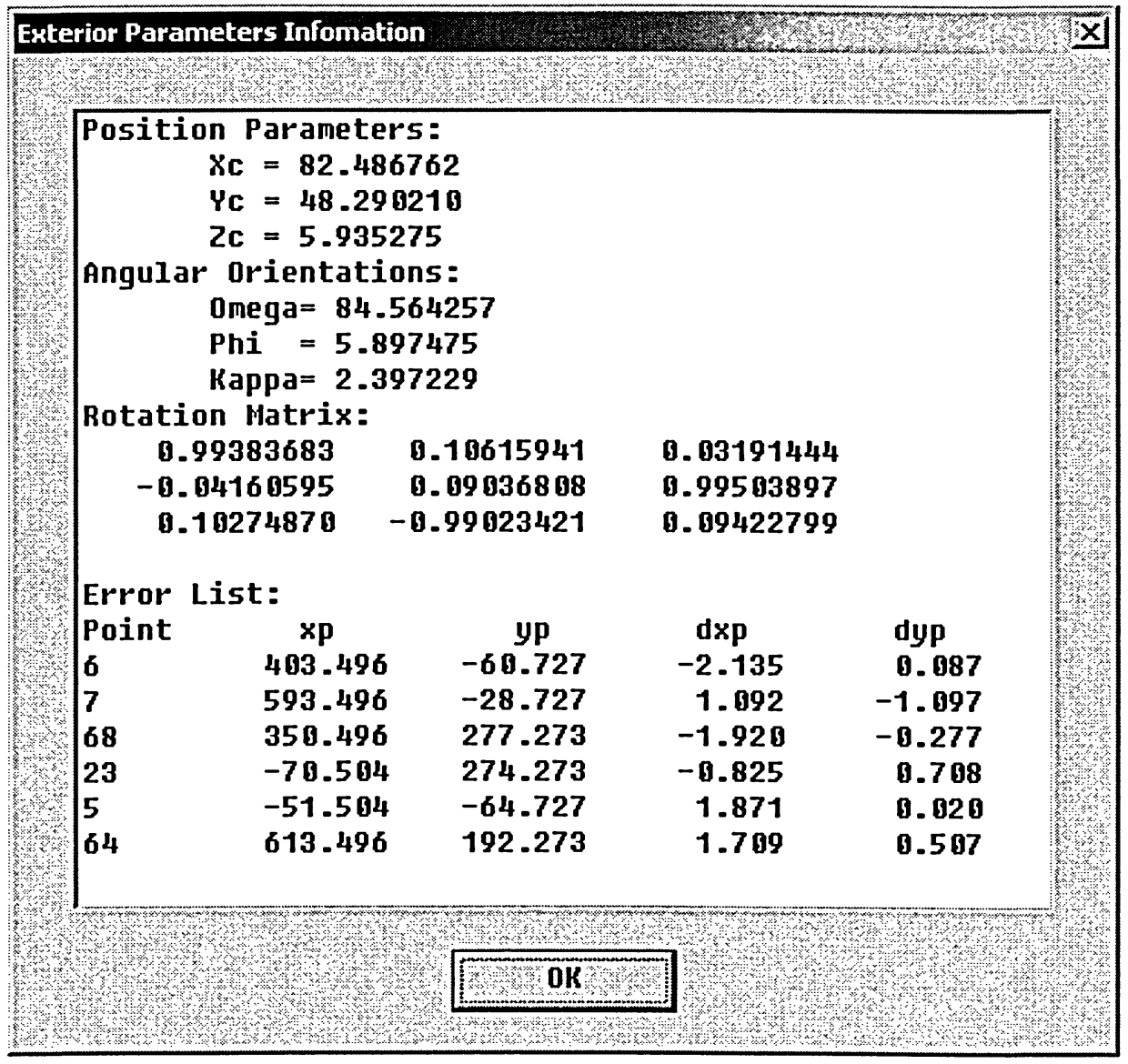

Figure 5-8 Results of external orientation

The external orientation program assists users when completing orientation operations.

The results of this program are used as input data for 3D reconstruction. 


\subsection{Chapter Summary}

Exterior elements determine the photograph position and angular orientation of image camera. The calculating process of exterior elements is called external orientation. Both GCPs and their corresponding image coordinates are required in external orientation. A new method for obtaining GCPs by using GIS and GPS technologies is proposed in Chapter 3. Sections 5.1 and 5.2 present two software programs used for realizing this method. The collected GCPs can be used in external orientation program. This program provides image coordinates measurement function and the computation of exterior elements. With known interior and exterior elements, ground coordinates of target objects are easy to calculate. 


\section{CASE STUDY OF 3D RECONSTRUCTION}

This chapter describes the study of 3D reconstruction using photogrammetric methods.

A software program is designed for this objective. It is a Microsoft Windows program coded by Visual $\mathrm{C}++$. There are 3 modules named model management, object measurement and graphics editing in this program. The details of above modules are described in the following sections. Figure 6-1 shows the main interface of this program.

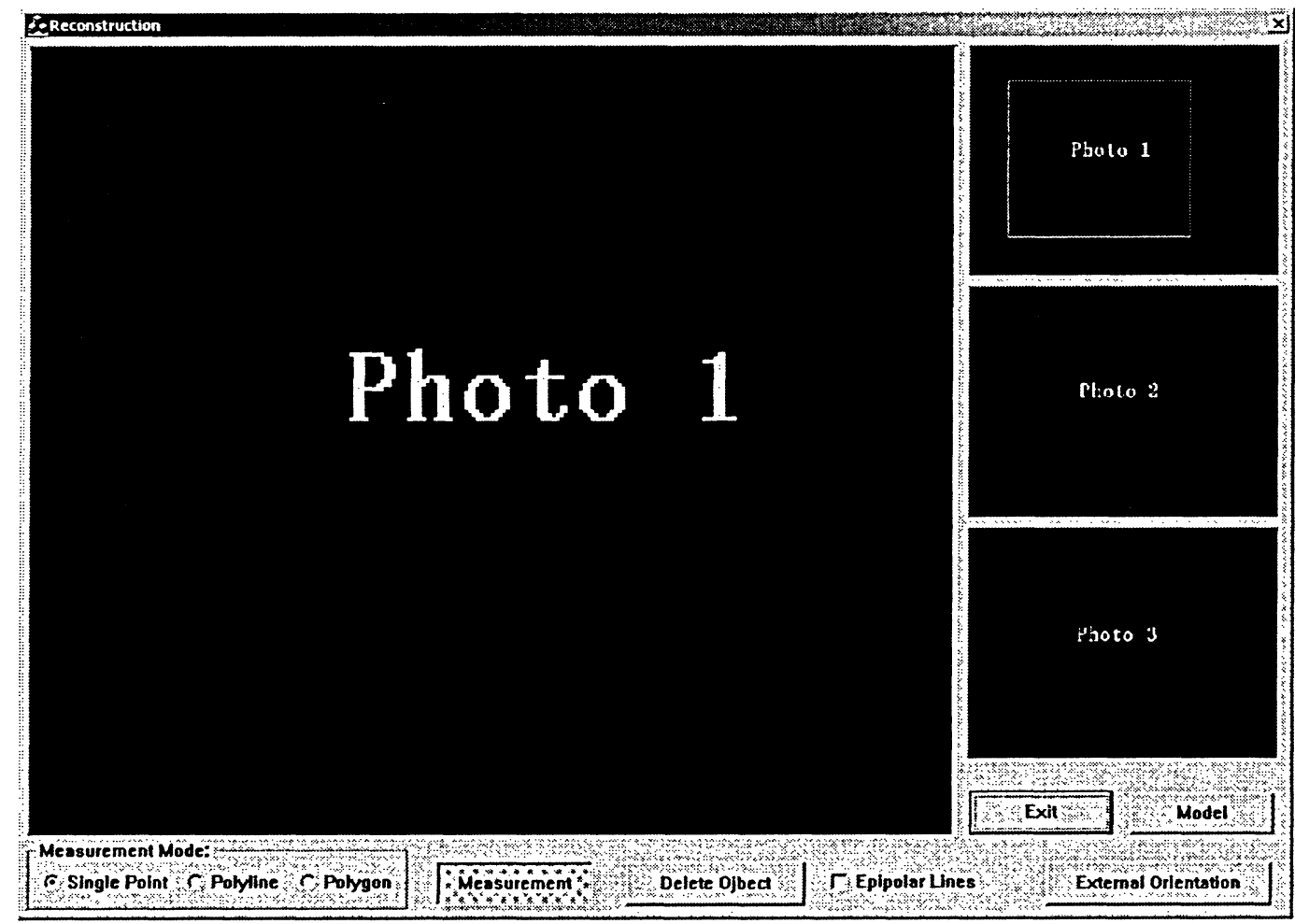

Figure 6-1 User interface of 3D reconstruction 


\subsection{Model Management Module}

This module is used for initializing, editing and saving model data. The term model in this program means a set of data including names of three digital images, map name, interior elements, exterior elements and fundamental matrix of three digital images. All of these data elements are needed for computing space coordinates of target object, using the photogrammetric methods.

As shown in Figure 6-2, a pop up window shows the names of 3 digital images and associated map. These names can be specified either in corresponding editing window or a "File open" window as character strings. These strings include the file paths and file names.

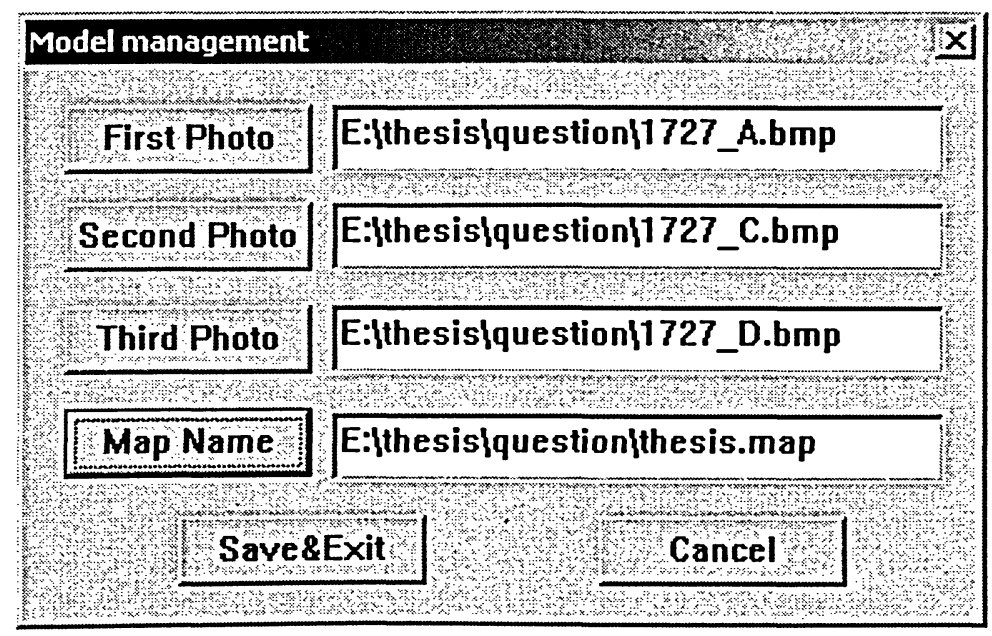

Figure 6-2 Model management window

An external orientation program loads the interior elements and computes the exterior 
elements. The orientation results are stored in a Visual $\mathrm{C}++$ structure. This structure is called "PPF_Struct" and is shown as follows:

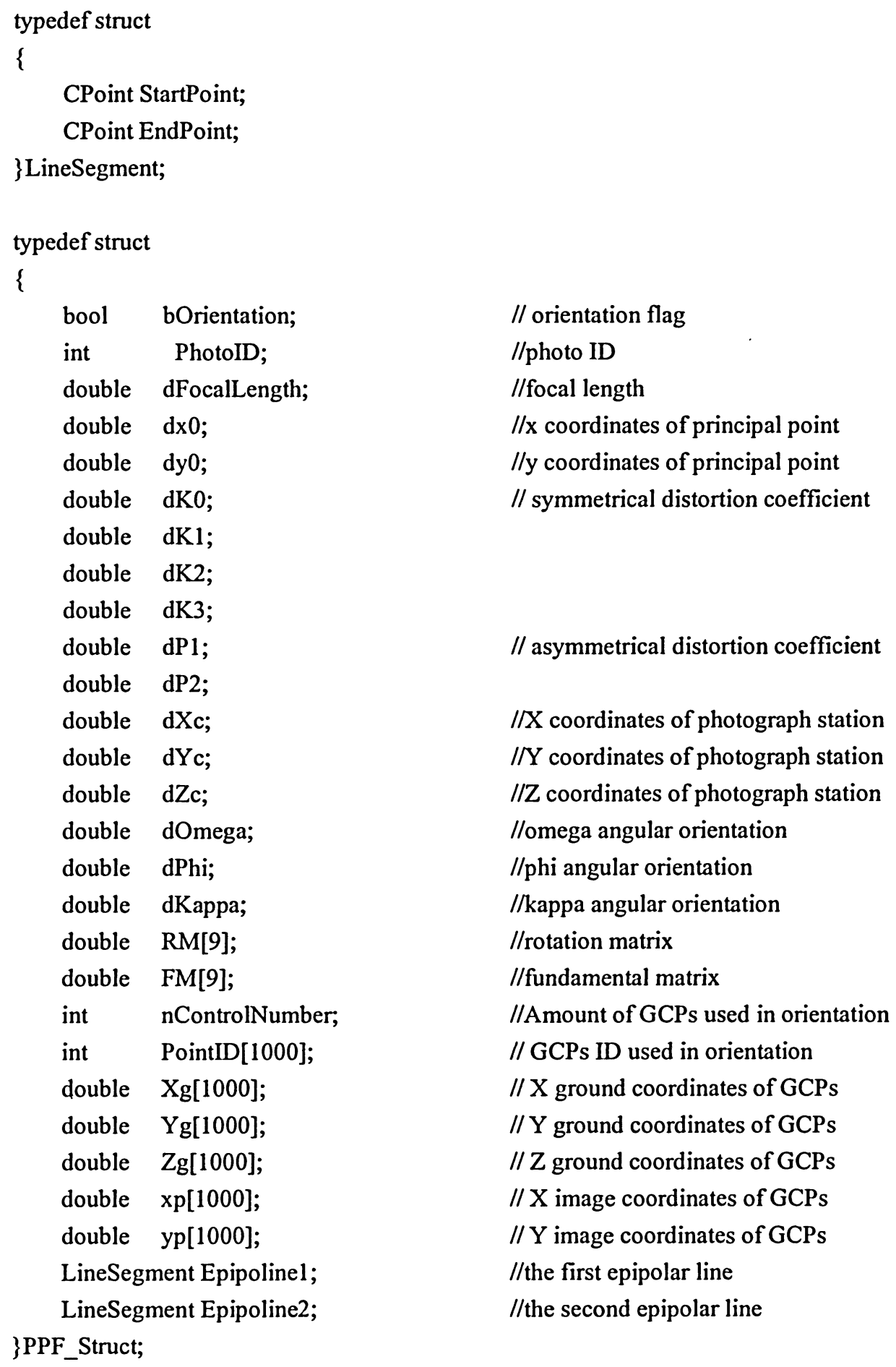

// orientation flag

//photo ID

//focal length

$/ / x$ coordinates of principal point

$/ / y$ coordinates of principal point

// symmetrical distortion coefficient

// asymmetrical distortion coefficient

I/X coordinates of photograph station //Y coordinates of photograph station //Z coordinates of photograph station //omega angular orientation $/ /$ phi angular orientation //kappa angular orientation //rotation matrix //fundamental matrix //Amount of GCPs used in orientation // GCPs ID used in orientation // X ground coordinates of GCPs // Y ground coordinates of GCPs $/ / \mathrm{Z}$ ground coordinates of GCPs // X image coordinates of GCPs // Y image coordinates of GCPs //the first epipolar line //the second epipolar line 
The model data set is automatically saved on a hard disk after the external orientation or model management operation. A C + class defined by the following code is used for loading and saving the model data.

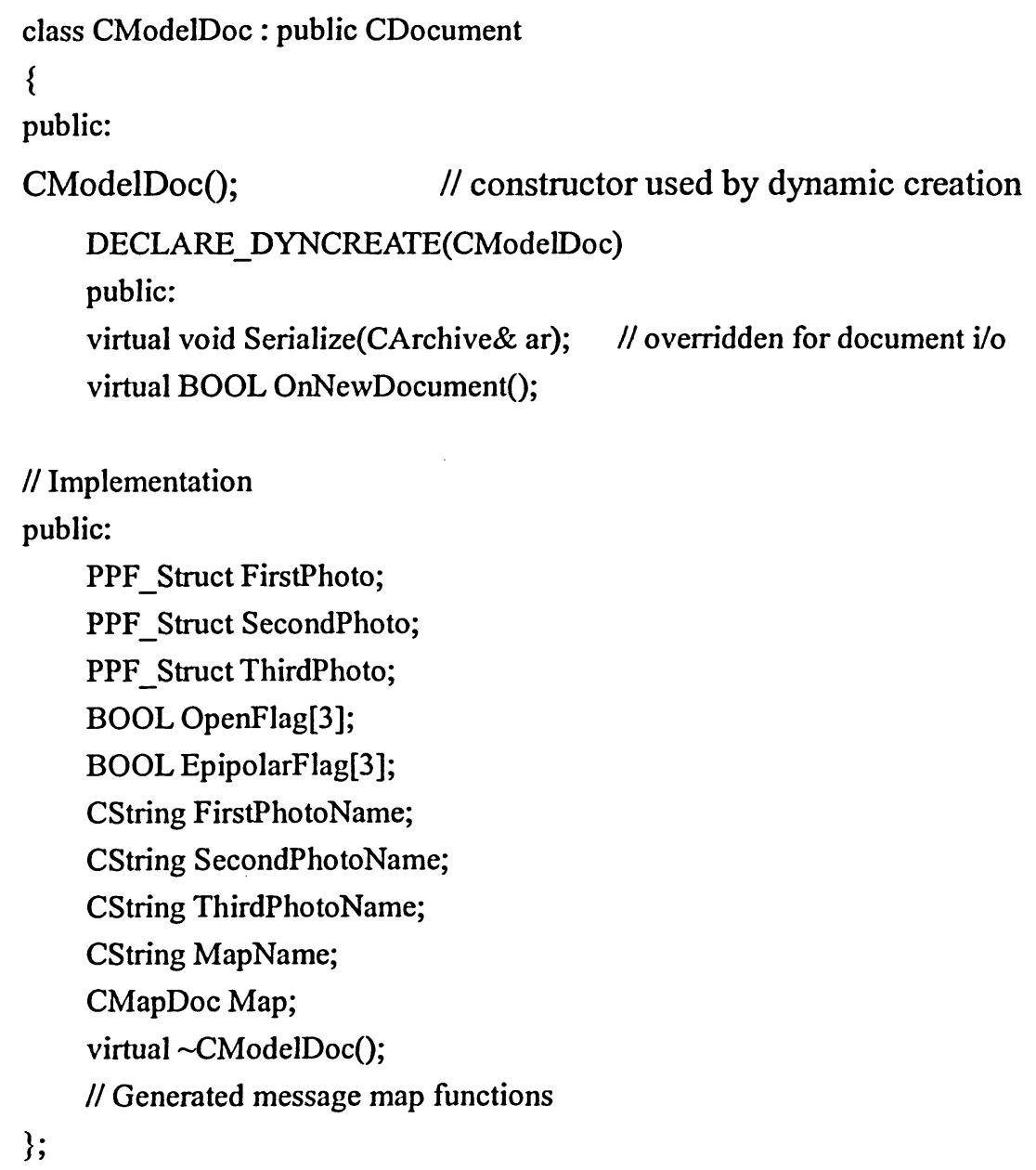

\subsection{Object Measurement Module}

With the model data mentioned in Section 6.1, the ground coordinates of the target object can be computed from the measured image coordinates. This module initially provides a measurement function that can be used for gaining image coordinates. As shown in Figure 6-3, there are four image windows in the program interface. Three 
small image windows display the scaled reductions of the three digital images. Although every image can be activated, only one image is active at a given time. A green rectangle displayed in each of these windows illustrates the range of active image shown in the large image window. The large image window shows a part of active digital image in a 1:1 scale. A pink cross shows the position of this point. Sometimes, the target object does not have a point feature. As such, it is difficult to determine the position of homologous points. Fortunately, homologous points are located in corresponding epipolar lines as mentioned in Section 3.3.4. Epipolar lines can be computed and displayed on all image windows as an assist function since, in most cases, they have intersections with this kind of target object. This assist function can be enabled or disabled in this program.

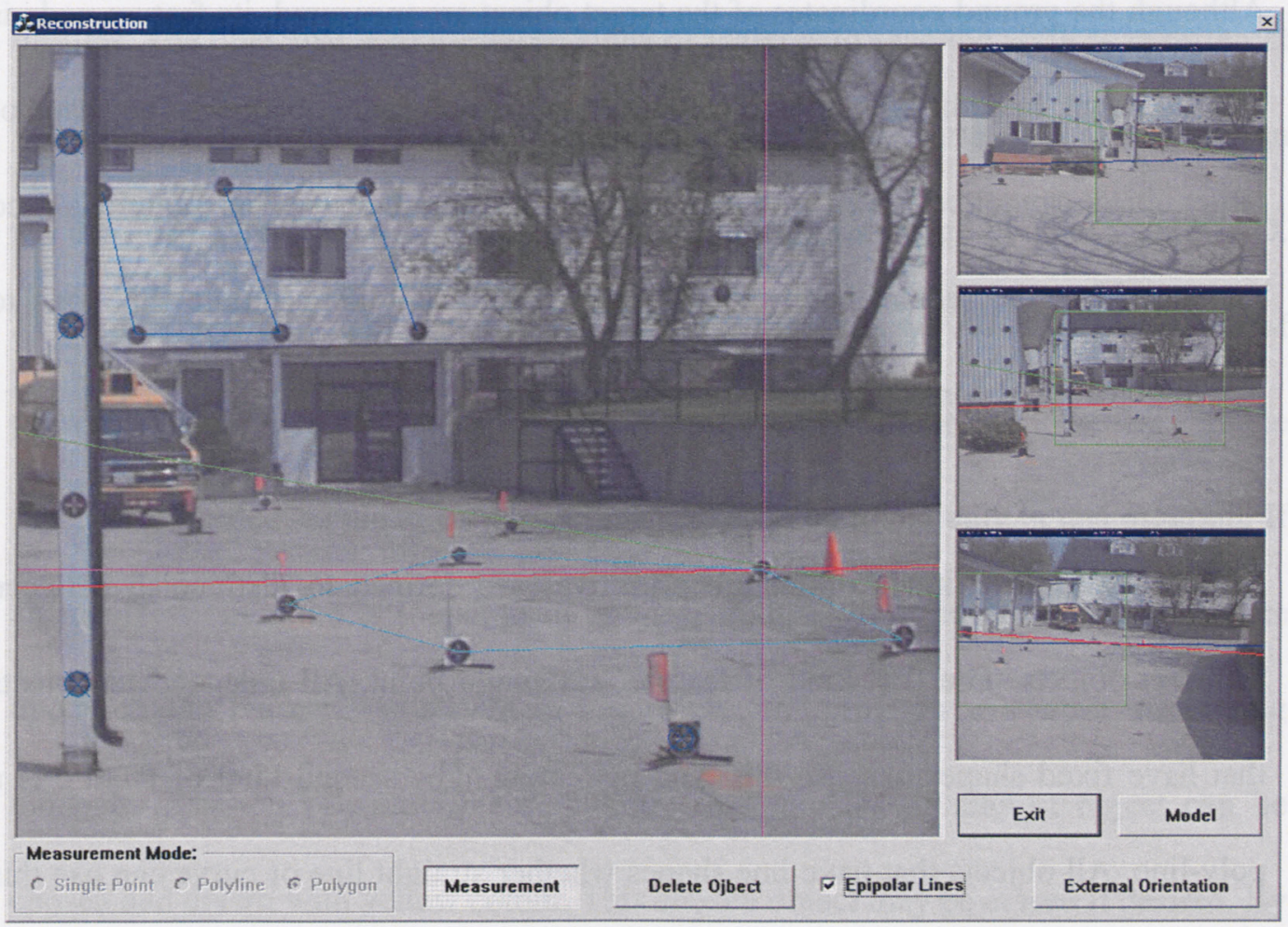

Figure 6-3 The interface of object measurement module. 
The image coordinates of target point can be measured by setting three digital images active, sequentially, and moving the pink cross on the target image point. Then the ground coordinates of target object can be computed. If just two of these three images overlap the target object, the ground coordinates are computed using space intersection. If all of three images overlap target object, a bundle adjustment is employed for calculating the ground coordinates. The results of space intersection using first two images are employed as approximations.

\subsection{Graphics Editing Module}

Although the ground coordinates of the target object are measured, its feature code is still unknown. In addition, only the vertices of target object are obtained. The shape of target object cannot be drawn without specifying its feature code. This module is used for specifying the feature code of target object and providing simple functions to drawn objects and correcting measurement mistakes.

In this study, three kinds of feature primitives are defined to distinguish between different objects. The first kind of feature is a single point. All independent objects that have fixed shapes can use this kind of feature. The second kind of feature is a poly-line. All objects that have line shapes whether straight line or curve can use this

kind of feature. The third kind of feature is a polygon. All objects that cover an area 
can use this kind of feature. This module provides a simple function to specify the features of target object. As shown in Figure 6-3, the feature can be chosen in "Measurement Mode" box when program is working in measurement mode. Figure 6-4 shows the legend of these kinds of features.

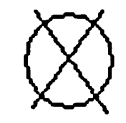

Single point

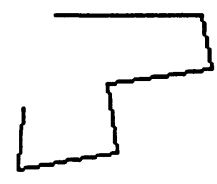

Poly-line

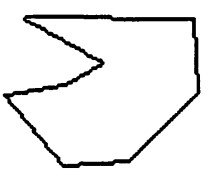

Polygon

Figure 6-4 The legend of three kinds of features

The three kinds of features defined above definitely cannot describe all underground utilities. The objective is to use these kinds of features to save the vertices in different ways in order that these data can be subsequently edited in AutoCAD. Operators can set the other information based on corresponding digital image by using AutoCAD. There is no need to make more kinds of editing functions, as AutoCAD is a kind of powerful graphics editing software with lots of special functions. However, a delete function is needed, as the measurement mistakes can be found only in this program. If an incorrect operation is found, such as measuring a wrong image point, the delete operation can erase it. As shown in Figure 6-5, the program will work in deleting mode by pressing "Delete Object" button. The graphics of interest object can be chosen and drawn with yellow colour. This chosen object may be erased if desired. By means of this function, the correctness of mapping objects is improved: 


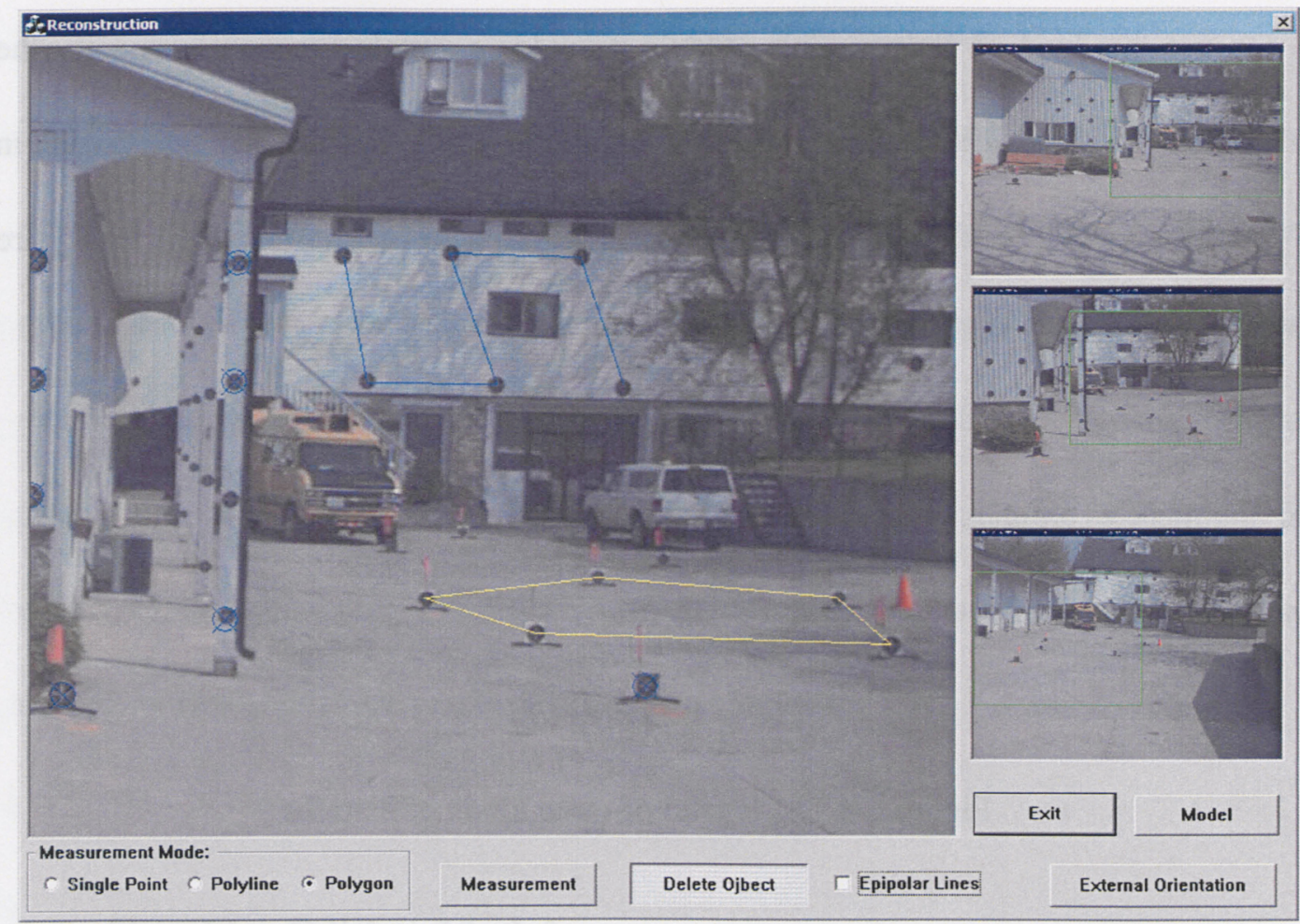

Figure 6-5 Delete a selected object

\subsection{Chapter Summary}

This chapter presents the solution of $3 \mathrm{D}$ reconstruction. A software program that consists of three modules is made for completing $3 \mathrm{D}$ reconstruction. The model management module is used for managing model data. This is a data preparation module that can be used to load, modify and save digital images, orientation data and graphics data. The object measurement module is used for measuring image coordinates from digital images and computing ground coordinates based on orientation data loaded in the management module. The graphics editing module is used for organizing measured ground coordinates by specifying different object 
features, displaying these objects on the corresponding digital image and deleting objects that contain mistakes. The 3D construction results are saved in graphics data file. If transformed to DXF format, they can be edited in.AutoCAD. 


\section{CONCLUSIONS AND RECOMMENDATIONS}

This study presents a photogrammetric solution to complete 3D reconstruction of underground utilities. In this chapter, the major achievements of the developed approach are summarized in Section 7.1. Some limitations are discussed in Section 7.2. In Section 7.3, conclusions are drawn based on previous discussions. Finally, recommendations for future research are given in Section 7.4.

\subsection{Summary of the Study}

Photogrammetry is a technology for obtaining reliable information about physical objects and the environment through processes of recording, measuring, and interpreting photographic images and phenomena. In this research work, a photogrammetric solution based on a set of software programs is developed to realize 3D reconstruction of underground utilities. This set of software programs can be divided into three parts, which are categorized as (1) camera calibrations programs, (2) GCPs collection and external orientation programs, and (3) 3D reconstruction program.

A commercial digital camera is employed to provide digital photographic images. Its interior elements and distortion coefficients are computed by using camera calibration programs. These programs consist of two programs. The first program is developed 
for obtaining image coordinates of corresponding GCPs where a special pattern is designed for providing 3D space coordinates. Different from traditional method, image matching and LSM techniques are employed in this program to detect such image coordinates based on the designed pattern. Image matching is used for automatically detecting the approximations of corresponding GCPs. These approximations help LSM detect accurate image positions of GCPs. The second program employs a DLT method to complete computation of the interior elements and lens distortion coefficients. These parameters can be saved in a LUT indexed by the corresponding actual focal length provided by the current digital camera. With this LUT, digital images captured under different focal lengths can be processed together. The distributions of distortions and resection errors are also displayed on the screen that help the operator check the results.

In order to obtain space position of the target object, the exterior elements of photographic images must be calculated. A group of GCPs appearing on digital images is needed in this calculating process. A new GCPs collection method that employs both GIS and GPS techniques is proposed in this study and realized in the GCPs collection software program. A GIS data transformation program is developed for transforming DXF format GIS data to our own graphics data format. An external orientation program provides not only a graphical interface to assist the operator when measuring image coordinates of corresponding GCPs from digital photographic images, but also the computation of the exterior elements. 
With interior elements, lens distortion coefficient and exterior elements, space coordinates of the target object can be solved if corresponding image coordinates of at least 2 digital images are measured. 3D reconstruction program is developed for loading digital images, managing corresponded parameters including interior and exterior element and distortion coefficients, measuring image coordinates of target object, calculating space coordinates and editing these space coordinates. The positions and shapes of underground utilities can be obtained in this program.

\subsection{Limitations of the Study}

Although the objective of this study is realized, there are still some limitations which are summarized as follows.

Firstly, the digital camera must provide the actual focal length in the camera calibration program because this value is used as an index in the LUT. If not, the camera can only work using a nominal focal length.

Secondly, the camera calibration program requires a good distribution of GCPs shown on the digital image because the calibration results are very sensitive to this distribution. Furthermore, in order to use the automatic combination function, the $\Phi$ and $\omega$ angular orientations should be very small. 
Thirdly, at least 3 GCPs are needed in the measurement area. Otherwise, external orientation cannot be easily processed. These GCPs must not be distributed on or near a straight line. In this case, erroneous results may be computed during external orientation.

Finally, in order to make the operation of 3D reconstruction straightforward, just three kinds of features, which are defined as single point, poly-line and polygon, are provided. The actual features of measured objects should be renamed, for example, a gas pipe, in AutoCAD program.

\subsection{Conclusions}

Nowadays, space information of underground utilities can be obtained by using different methods as mentioned in Chapter 2 . The common disadvantages of all these methods are the slow speed, high cost and the fact that they could not be used during the construction of the underground utilities. Based on digital images, a photogrammetric method provides a high accuracy and fast 3D reconstruction tool for mapping exposed underground utilities. This thesis has presented a solution of obtaining space information of underground utilities using photogrammetric methods. Several findings and conclusions are summarized below: 
First, Commercial digital cameras can be used for providing original digital images. An automatic detection program provides an automatic method to obtained image coordinates of GCPs. Based on these coordinates, the camera calibration program computes not only the interior elements of digital cameras but also the lens distortion coefficients. The interior elements include the focal length and the position of the principal point. These elements are the basic parameters of camera interior geometry in photogrammetry. After correcting the lens distortion, the accuracy of these images satisfies photogrammetric requirement. A LUT lets digital cameras work within different focal lengths if the corresponding actual focal lengths are provided.

Second, Collecting GCPs from an existing GIS data set by using GPS receiver is effective. GCPs collection programs provide corresponding functions and make it easy to complete this collection. Based on the collected GCPs, the exterior elements of digital images can be calculated in an external orientation program.

Third, Spatial information of target object can be obtained in the 3D reconstruction program. In this program, space coordinates are computed after corresponding image coordinates are measured where the feature of target object is also determined. These data are also drawn on the images that help operators easily check incorrect operations. A deleting function can assist in finding and deleting erroneous data. These space data are saved in a graphics file and can be 
transformed to a DXF file for further editing in AutoCAD software.

\subsection{Recommendations for Future Research}

Based on the proposed methodology and current research works, some recommendations can be summarized in order to extend the study in the future.

First, Digital image matching technique can be used in 3D reconstruction. This technique will help operators easily find homologous points within digital images. However, based on the methodology of image matching, this locating task will not be successful all of the time. Hence, it should be used as an aiding function.

Second, Automatic line and edge detection is recommended in 3D reconstruction. Most underground utilities have line or edge features. It is easy to extract such features from digital images by using automatic line and edge detection method. Operators simply have to choose target objects from the detection results.

Third A transformation software program should be developed for transforming graphics files to DXF format. Without this program, the graphics file cannot be edited again in AutoCAD. 


\section{BIBLIOGRAPHY}

Abdel-Aziz, Y.I. and H.M. Karara, 1971. Direct linear transformation into object space coordinates in close-range photogrammetry, Proceedings of the Symposium on Close-Range Photogrammetry, Urbana-Champaign, IL, January 1971, pp. 1-18.

Ahmed, M. and A. Farag, 2001. Non-metric calibration of camera lens distortion, Proceedings of the International Conference on Image Processing, Thessaloniki, Greece, September 2 (2001), pp. 157-160.

Ahn, S.J., W. Rauh, and S. I. Kim, 2001. Circular coded target for automation of optical 3D-measurement and camera calibration, International Journal of Pattern Recognition and Artificial Intelligence, 15(6): 905-919.

Anspach, J. H., and Stanley E. Wilson, 1994. A case study of an underground $138 \mathrm{KV}$ transmission line design utilizing subsurface utility engineering, Proceedings of the 56th Annual American Power Conference, 56:(1): 37-141.

Anspach, J. H, 1997. Design and Construction risk management for existing utilities, Construction Congress V, pp. 301-306.

Anspach, J. H, 1995. Subsurface utility engineering: upgrading the quality of utility information, International Conference on Advances in Underground Pipeline Engineering-Proceedings, pp. 813-824.

Anspach, J. H., 1996. Integrating Technology into Subsurface Utility Engineering Projects, Pipeline \& Gas Journal, 223(6):. 41-42.

AutoCAD DXF Reference, Autodesk, Inc, 2005.

Bacakoglu, H. and S. Kamel, 1997. A tree-step camera calibration method, IEEE Transactions on Instrumentation and Measurement, 46 (5): 1165-1172.

Baillard, C., and O. Dissard, 2000. A stereo matching algorithm for urban digital elevation models, Photogrammetric Engineering \& Remote Sensing, 66(9): 1119-1128.

Bragin, S.I., I.V. Bragin, V.P. Sgibnev, S.E. Chadov, V.I. Gusevsky, Y.B. Bragina, A.A. Morozov, and V.V. Tsutskov, 2000. Remote detection of objects in soil using a microwave and IR scanner, Internationl Conference on Microwave and Millimeter Wave Technology Proceedings, pp. 607-610. 
Brown, D.C., 1971. Close-range camera calibration, Photogrammetric Engineering, 37(8): 855-866.

Caldecott, R., M. Poirier, D. Scofea, D.E. Svoboda, and A.J. Terzuoli, 1988. Underground mapping of utility lines using impulse radar, IEE Proceedings, 135 (4): 343-353.

Chua, C., Y. Ho, and Y. Liang, 2000. Rejection of mismatched correspondences along the affine epipolar line, Image and Vision Computing, Vol.18, pp. 445-462.

Chen, Q., H. Wu, and T. Wada, 2004. Camera calibration with two arbitrary coplanar circles, Proc. European Conf. Computer Vision, Vol. 3, pp. 521-532.

DeAgapito, L., E. Hayman, and I.D. Reid, 2001. Self-calibration of rotating and zooming cameras, International Journal of Computer Vision, 45 (2): 107-127.

Faugeras, O.D., L. Quan, and P. Sturm, 2000. Self-calibration of a 1D projective camera and its application to the self-calibration of a $2 \mathrm{D}$ projective camera, IEEE Transactions on Pattern Analysis and Machine Intelligence, 22 (10): 1179-1185.

Fitzgibbon, A. W., 2001. Simultaneous linear estimation of multiple view geometry and lens distortion, Proceedings of the IEEE Conference on Computer Vision and Pattern Recognition (CVPR'01), Kauai, HI, December 1 (2001), pp. $125-132$.

Gonzalez, R. C. and R. E. Woods, 2001. Digital Image Processing, Prentice-Hall, Upper Saddle River, New Jersey, 793p.

Gülch, E., 1991. Results of test on image matching of ISPRS WG III/4, ISPRS Journal of Photogrammetry and Remote Sensing, 46(1): 1-8.

Handlon, B., S.J. Lorenc, L. Bernold, and G. Lee, 2000. Tool integrated electromagnetic pulse induction technology to locate buried utilities, ISCAS IEEE International Symposium on Circuits and Systems, May 28-31, Geneva, Swizerland, pp. 529-532.

Hansen, T., M. Oristaglio, D. Miller, M. Burns, A. Derubeis, P. Albats, R. Casadonte, R. Deming, R. Birken, and J. Haldorsen, 2005. Effectient large-scale underground utility mapping wit a multi-channel ground-penetrating radar system, URL: http://www.ursi.org/Proceedings/ProcGA02/papers/p1137.pdf, (last accessed: July 30, 2005).

Heikkila, J. 2000. Geomatric camera calibration using circular control points, IEEE 
Trans. Pattern Analysis and Machine Intelligence, 22(10): 1066-1077.

Hu, R. and Q. Ji, 2001. Camera self-calibration from ellipse correspondences, Proc. IEEE International Conference, Robotics and Automation, Vol. 3, pp. 2191-2196.

Jenus, J. and K. Bakhtar, EarthRadar application for detecting/mapping underground utilities, $2005 . \quad$ URL: http://www.federallabs.org/utilities/Presentations/EarthRadar for Underground Utilities_Mapping_Bakhtar.pdf, (last accessed: July 30, 2005).

Jeong, H. S., D. M. Abraham, and J. J. Lew, 2004. Evaluation of an emerging market in subsurface utility engineering, Journal of Construction Engineering and Management, ASCE, March/April, pp. 225-234.

Jokinen, O., 1998. Area-based matching for simultaneous registration of multiple 3-D profile maps, Computer Vision and Image Understanding, 71(3): 431-447.

Kim, J., P. Gurdjos, and I. Kweon, 2005. Geometric and algebraic constraints of projected concentric circles and their application to camera calibration, IEEE Transaction on Pattern Analysis and Machine Intelligence, 27(4): 637-642.

Kim, J.S., H.W. Kim, and I.S. Kweon, 2002. A camera calibration method using concentric circles for vision applications, Proc. Asian Conf., Computer Vision, Vol. 2, pp. 515-520.

Lavest, J.M., M. Viala, and M. Dhome, 1998. Do we really need an accurate calibration pattern to achieve a reliable camera calibration? Proceedings of the European Conference on Computer Vision (ECCV'98), Freiburg, Germany, pp.158-174.

Lenz, R.K., and R.Y. Tsai, 1988. Techniques for calibration of the scale factor and image center for high accuracy $3 \mathrm{D}$ machine vision metrology, IEEE Transactions on Pattern Analysis and Machine Intelligence, 10(5): 713-720.

Lew, J. J., 1996. Subsurface utility engineering: An initial step in project development, ASC Proceedings of the 32nd Annual Conference, College Station, Texas, pp. 217-222.

Li, M. and J.M. Lavest, 1996. Some aspects of zoom lens camera calibration, IEEE Transactions on Pattern Analysis and Machine Intelligence, 18(11): 1105-1110.

Liang, T., and C. Heipke, 1996. Automatic relative orientation of aerial images, Photogrammetric Engineering \& Remote Sensing, 62(1): 47-55. 
Lucchese, L., 2005. Geometric calibration of digital cameras through multi-view rectification, Image and Vision Computing, Vol 23, pp. 517-539.

Lucchese, L., 2003. Estimating the pose and focal length of a camera from the perspective projection of a planar calibration plate, Proceedings of the Fifth IASTED International Conference on Signal and Image Processing (SIP 2003), Honolulu, Hawaii, August 2003, pp.201-206.

Lucchese, L., 2003. Digital camera calibration. Part I: internal and external geometry from multi-view alignment, Proceedings of the 3rd IASTED International Conference on Visualization, Imaging, and Image Processing (VIIP 2003), Benalma'dena, Spain, September III (2003), pp. 1061-1065.

Lue, Y. and K. Nova, 1991. Recursive grid- dynamic window matching for automatic DEM generation, GIS/LIS ACSM-ASPRS Fall Convention, 1991, pp. A254-A260.

Luong, Q.T., and O.D. Faugeras, 1997. Self-calibration of a moving camera from point correspondences and fundamental matrices, International Journal of Computer Vision, 22 (3): 261-289.

Madani, E. M., J. S. Bethel, and J.D. McGlone, 2001. Introduction to Modern Photogrammetry, John Wiley \& Sons, New York, NY, 479 p.

Malis, E. and R. Cipolla, 2002. Camera self-calibration from unknown planar structures enforcing the multiview constraints between collineations, IEEE Transactions on Pattern Analysis and Machine Intelligence, 24(9): 1268-1272.

Maybank, S. and O.D. Faugeras, 1992. A theory of self calibration of a moving camera, International Journal of Computer Vision, 8 (2): 123-151.

Mcintosh, J. H. and K. M. Mutch, 1988. Matching straight lines, Computer Vision Graph Image Processing 43, pp. 368-408.

Medioni, G., and R. Nevatia, 1984. Matching image using linear features, IEEE Transactions Pattern and Mach Intell, Vol. 6, pp 675-785.

Medinoi, G. and R. Nevatia, 1985. Segment based stereo matching, Computer Vision Image Processing, Vol. 31, pp. 2-18.

Meng, X. and Z. Hu, 2003. A new easy camera calibration technique based on circular points, Pattern Recognition, 36(5): 1155-1164.

Mount, D. M., N. S. Netanyahu, and J. L. Moigne, 1999. Efficient algorithm for 
robust feature matching, Pattern Recognition, Vol. 32, pp. 17-38.

O'Neill, M. and M. Denos, 1996. Automated system for coarse-to-fine pyramidal area correlation stereo matching, Image and Vision Computing, 14(3): 225-236.

Otto, G. P., 1988. Rectification of SPOT data for stereo image-matching, International Archives of Photogrammetry and Remote Sensing, 27(B3): 635-645.

Otto, G., and T. Chau, 1989. A region growing algorithm for matching of terrain image, Image and Vision Computing, Vol. 7, pp. 83-94.

Pollefeys, M., R. Koch, and L. Van Gool, 1998. Self-calibration and metric reconstruction inspite of varying and unknown intrinsic camera parameters, International Journal of Computer Vision, 32 (1): 7-25.

Sturm, P.F. and S.J. Maybank, 1999. On plane-based camera calibration: a general algorithm, singularities, applications, Proceedings of the IEEE Conference on Computer Vision and Pattern Recognition (CVPR'99), Fort Collins, CO, June 1 (1999), pp. 23-25.

Sturm, P.F., 1997. Self-calibration of a moving zoom-lens camera by precalibration, Image and Vision Computing, Vol.15, pp. 583-589.

Swaminathan, R. and S.K. Nayar, 2002. Nonmetric calibration of wide-angle lenses and polycameras, IEEE Transactions on Pattern Analysis and Machine Intelligence, 22 (10): 1172-1178.

Tsai, R., 1987. A versatile camera calibration technique for high accuracy 3D machine vision metrology using off-the-shelf TV cameras and lenses, IEEE $J$. Robotics and Automation, 3(4): 323-344.

Tulloch, M, 2005. Proposed solution for mapping underground utilities for buried asset management, Internal Report, 37p.

Tulloch, $\mathrm{M}$ and W. Hu, 2005. A proposed solution for mapping underground utilities for buried asset management, Ryerson Graduate Student Research Article Competition 2005, 4p.

Valkenburg, R.J., 1996. Camera calibration using multiple references, In Image and Vision Computing New Zealand, pp. 61-66.

Veneziano, D., S. Hallmark, and R. Souleyrette, 2002. Comparison of LIDAR and conventional mapping methods for highway corridors studies, Final Report of 
Center for Transportation Research and Education, October, 2002, 58p.

Wang, Z., 1990. Principle of Photogrammetry: with Remote Sensing, Press of Wuhan Technical University of Surveying and Mapping and Publishing House of Surveying and Mapping, Wuhan, China, 575p.

Weng, J., P. Cohen, and M. Herniou, 1992. Camera calibration with distortion models and accuracy evaluation, IEEE Transactions on Pattern Analysis and Machine Intelligence, 14 (10): 965-980.

Wolf, P. R. and B. A. Dewitt, 2000. Elements of Photogrammetry: with Application in GIS, $3^{\text {rd }}$ edition, McGraw-Hill, New York, 608p.

Wong, K.Y., P.R.S. Mendonca, and R. Cipolla, 2003. Camera calibration from surfaces of revolution, IEEE Transactions on Pattern Analysis and Machine Intelligence, 25 (2): 147-161.

Wu, Y., H. Zhu, Z. Hu, and F. Wu, 2004. Camera calibration from quasi-affine invariance of two parallel circles, Proc. European Conf. Computer Vision, Vol. 1, pp. 190-202.

Xie, P., 2004. A Web-based 3D Visualization Prototype System for High-resolution Satellite Colour Stereo Images. M.Sc.E. thesis, University of New Brunswick pp.31-32

Yang, C., F. Sun, and Z. Hu, 2000. Planar conic based camera Calibration, Proc Int'l Conf. Pattern Recognition, Vol. 1, pp. 555-558.

Zembillas, N. M., 2003. Subsurface utility engineering: A technology-driven process that results in increased safety, fewer claims, and lower costs. Pipelines, Vol. 2, pp. 1422-1428.

Zhang, Z., 1999. Flexible camera calibration by viewing a plane from unknown orientations, Proceedings of International Conference on Computer Vision (ICCV'99), Corfu, Greece, September 1999, pp. 666-673.

Zhang, Z., R. Deriche, O. Faugeras, and Q. Luong, 1995. A robust technique for matching the uncalibrated images through the recovery of the unknown epipolar geometry, Artificial Intelligence, Vol. 78, pp. 87-119.

Zhang, Z., J. Zhang, M. Liao, and L. Zhang, 2000. Automatic registration of multi-source imagery based on global image matching, Photogrammetric Engineering \& Remote Sensing, 66 (5): 625-629.

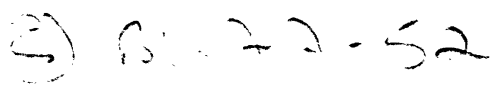

